Propriedades aritméticas e topológicas de uma classe de Fractais de Rauzy 



\title{
Propriedades aritméticas e topológicas de uma classe de Fractais de Rauzy ${ }^{1}$
}

\author{
Tatiana Miguel Rodrigues
}

Orientador: Prof. Dr. Daniel Smania Brandão

Co-orientador: Prof. Dr. Ali Messaoudi

Tese apresentada ao Instituto de Ciências Matemáticas e de Computação da Universidade de São Paulo, como parte dos requisitos para obtenção do título de Doutor em Ciências - Área: Matemática.

USP - São Carlos

Janeiro/2010

\footnotetext{
${ }^{1}$ Este trabalho teve suporte financeiro do CAPES
} 

Aos meus pais. 



\section{Agradecimentos}

Agradeço ao Deus conosco pela benção de mão consciente, por todo o tempo estar presente em minha vida, me direcionando e me aprumando.

Agradeço pela colaboração dos professores Prof. Dr. Ali Messaoudi e ao Prof. Dr. Daniel Smania Brandão, que a graça esteja sempre presente em suas vidas. Agradeço pela paciência e atenção, pelo tempo dedicado, pelas respostas às dúvidas. Muito agradecida!

Agradeço aos meus pais, Ruben e Suely, e irmãos Nayhana e Vladimir, por todo o apoio, paciência, incentivo e todo o amor e ao meu namorado Thiago por todo o carinho, apoio e atenção.

Aos docentes do departamento de matemática do ICMC e da UNESP por todo os ensinamentos. Em especial ao Prof. Dr. Jéfferson Luiz Rocha Bastos pela atenção e paciência.

Aos funcionários do ICMC por estarem sempre à disposição e tornarem nossa vivência mais agradável. E em especial às funcionárias da Pós-Graduação, Beth, Laura, Ana Paula, Lívia e Glaucia.

Aos amigos do doutorado.

À CAPES pelo apoio financeiro.

Agradeço à todos que colaboraram para a realização deste trabalho. 



\section{Resumo}

Estudamos as propriedades aritméticas, geométricas e topológicas de uma classe dos chamados Fractais de Rauzy. Estudamos particularmente o azulejamento periódico do plano complexo $\mathbb{C}$ induzido por eles, assim como a dimensão de Hausdorff de suas fronteiras. Tal trabalho exige um estudo detalhado da fronteira destes conjuntos, que está associada às propriedades aritméticas da $\alpha$-representação dos números complexos com respeito a um certo número algébrico $\alpha$. 



\section{Abstract}

We study the arithmetic, geometric and topological properties of a class of the so-called Rauzy's fractals. In particular we study the periodic tiling of the complex plane $\mathbb{C}$ induced by them and the Hausdorff dimension of its boundary. Such work is connected to a detailed study of the boundary of such sets and the arithmetic properties of the $\alpha$ representation of complex numbers with respect to a certain algebraic number $\alpha$. 



\section{Sumário}

1 Sistemas de Numeração $\quad 1$

$1.1 \beta$-Numeração . . . . . . . . . . . . . . . . . . . . . . 1

1.2 Sistema de Numeração sobre os inteiros . . . . . . . . . . . . . . . . . 4

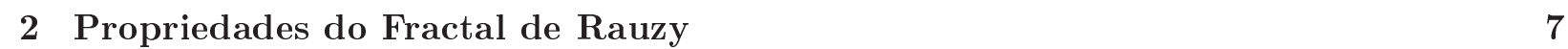

2.1 Propriedades do Fractal de Rauzy . . . . . . . . . . . . . . . . . . . . . 7

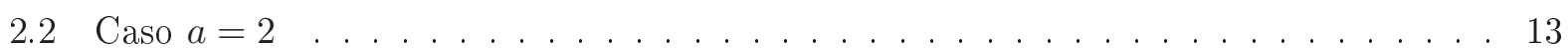

$\begin{array}{lll}3 & \text { O autômato que reconhece a fronteira de } \mathcal{R} & 17\end{array}$

3.1 Relação com as representações impróprias na base $\alpha \ldots \ldots$. . . . . . . . . 17

3.1.1 Definição do autômato que reconhece os pontos com no mínimo duas expansões 18

3.2 Caracterização dos pontos com duas expansões . . . . . . . . . . . . . . . . 19

4 Aplicação do autômato para a parametrização da Fronteira de $\mathcal{R} \quad 31$

4.1 Parametrização de $\mathcal{R}_{\alpha-1} \ldots \ldots \ldots \ldots \ldots \ldots \ldots$. . . . . . . . . . . . . . . . . . . . . . . . . . . .

4.2 Cálculo da dimensão de Hausdorff . . . . . . . . . . . . . . . . . . . . . . . . 49

$\begin{array}{lll}5 & \text { Parametrização da fronteira de } \mathcal{F} & 51\end{array}$

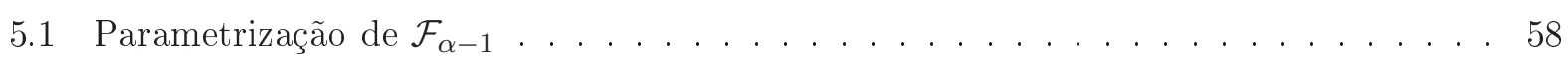

5.2 Cálculo da dimensão de Hausdorff . . . . . . . . . . . . . . . . . . . . . 60

6 Apêndice $\quad 63$

$\begin{array}{ll}\text { Referências Bibliográficas } & 75\end{array}$ 



\section{Introdução}

O objetivo desta tese é estudar as propriedades topológicas, geométricas e aritméticas de uma classe de Fractais de Rauzy.

O Fractal de Rauzy é um subconjunto do plano complexo que foi definido por G. Rauzy em 1982 ([26]). Este conjunto foi estudado por vários matemáticos (ver [1], [6], [15], [16], [18], [30]) e relacionado com diversas áreas da Matemática como sistemas dinâmicos, teoria dos números entre outras. Em particular, sabe-se que ele é um conjunto compacto, conexo, com interior simplesmente conexo e sua fronteira é uma curva de Jordan fractal. Além do mais ele induz um azulejamento periódico do plano complexo.

Existem vários métodos para se construir o Fractal de Rauzy, um deles é via Sistemas de Numeração, e é feito da seguinte maneira:

Seja $\left(T_{n}\right)_{n \geq 0}$ a sequência tal que $T_{0}=T_{1}=0, T_{2}=1$, e satisfazendo a recorrência linear

$$
T_{n+3}=T_{n+2}+T_{n+1}+T_{n}, n \geq 0 .
$$

Sabe-se, usando o algoritmo "fominha"(algoritmo Greedy) que todo inteiro natural se escreve de uma maneira única como $n=\sum_{i=3}^{N} \varepsilon_{i} F_{i}$ onde os dígitos $\varepsilon_{i}$ satisfazem a seguinte propriedade: $\varepsilon_{i} \in\{0,1\}$ e $\varepsilon_{i} \varepsilon_{i+1} \varepsilon_{i+2} \neq 111, \forall i \geq 3$, ou equivalentemente, $\varepsilon_{i} \varepsilon_{i-1} \varepsilon_{i-2}<_{\text {lex }} 111$ para todo $i \geq 5$ onde $<_{l e x}$ é a ordem lexicográfica.

Considere o polinômio $P(x)=x^{3}-x^{2}-x-1$. É fácil verificar que $P(x)$ possui uma raiz $\beta>1$ e duas raízes complexas $\alpha$ e $\bar{\alpha}$ de módulo menor que 1 .

O Fractal de Rauzy é o conjunto $\mathcal{E}=\left\{\sum_{i=3}^{\infty} \varepsilon_{i} \alpha^{i}, \varepsilon_{i} \in\{0,1\}\right.$ e $\left.\varepsilon_{i} \varepsilon_{i-1} \varepsilon_{i-2}<_{\text {lex }} 111, \forall i \geq 5\right\}$. $([26])$

A fronteira de $\mathcal{E}$ foi objeto de estudos de vários matemáticos. Em particular, em [30] Kimura e Ito calcularam a dimensão de Hausdorff da fronteira de $\mathcal{E}$. Em [18] Messaoudi mostrou que a fronteira de $\mathcal{E}$ é gerada por um automato finito. Usando este fato, ele provou várias propriedades da fronteira de $\mathcal{E}$ como o fato que ela é uma curva de Jordan e um quasi-círculo. 


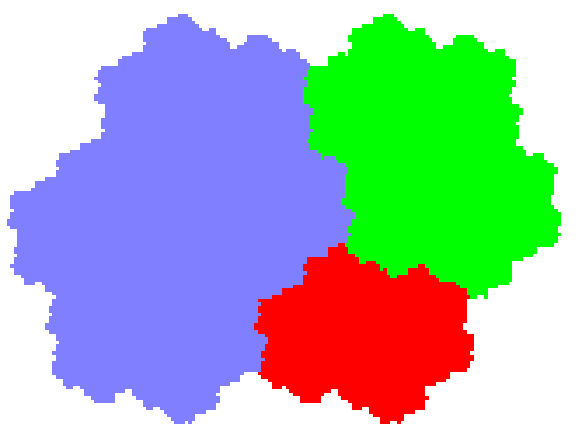

Figura 1: Fractal de Rauzy

Messaoudi [20] estendeu esse resultado para certos conjuntos $\mathcal{E}_{a}, a \geq 2$ associados aos polinômios $Q_{a}(x)=x^{3}-a x^{2}-x-1$.

Neste trabalho vamos considerar os polinômios do tipo $P_{a}(x)=x^{3}-a x^{2}+x-1$, onde $a \geq 2$. Pode-se provar que (ver Proposição 6.0.1, anexo) $P_{a}(x)$ possui uma raiz real $\beta>1$ e duas raízes complexas $\alpha$ e $\bar{\alpha}$ conjugadas e com módulo menor que 1 (ver [1]). De modo análogo ao Fractal de Rauzy clássico podemos associar ao polinômio $P_{a}(x)$ o conjunto (Fractal de Rauzy) definido da seguinte maneira:

Seja $\left(R_{n}\right)_{n \geq 0}$ a sequência recorrente definida por: $R_{0}=1, R_{1}=a, R_{2}=a^{2}-1, R_{n+3}=$ $a R_{n+2}-R_{n+1}+R_{n}, \forall n \geq 0$ onde $a \in \mathbb{N}, a \geq 2$.

Usando o algoritmo "fominha", podemos provar (ver Proposição 1.2.1, Capítulo 1) que todo inteiro natural se escreve de maneira única como $n=\sum_{i=0}^{N} \varepsilon_{i} R_{i}$ onde a sequência $\left(\varepsilon_{i}\right)_{0 \leq i \leq N}$ satisfaz a condição:

$\varepsilon_{i} \varepsilon_{i-1} \varepsilon_{i-2} \varepsilon_{i-3}<_{\text {lex }}(a-1)(a-1) 01, \forall i \geq 5, \varepsilon_{0}<_{\text {lex }} a, \varepsilon_{1} \varepsilon_{0}<_{\text {lex }}(a-1)(a-1), \varepsilon_{2} \varepsilon_{1} \varepsilon_{0}<_{\text {lex }}(a-1)(a-1) 0$

onde $<_{\text {lex }}$ é a ordem lexicográfica. Denotaremos por $\mathcal{M}$ o conjunto formado pelas sequências que satisfazem tais condições.

A sequência $\left(\varepsilon_{i}\right)_{0 \leq i \leq N}$ é chamada representação de $N$ na base $\left(R_{n}\right)$ ou simplesmente uma $R$ representação.

Definimos o Fractal de Rauzy associado a $\left(R_{n}\right)$ por

$$
\mathcal{R}_{a}=\left\{\sum_{i=2}^{\infty} \varepsilon_{i} \alpha^{i}, \forall N \geq 0,\left(\varepsilon_{i}\right)_{2 \leq i \leq N} \text { é uma R-representação }\right\} .
$$

O conjunto $\mathcal{R}_{a}$ é chamado fractal de Rauzy associado a sequência $\left(R_{n}\right)$ ou simplesmente fractal de Rauzy com restrições. 
Por outro lado considerando a sequência $\left(G_{n}\right)_{n \geq 0}$ definida por $G_{0}=1, G_{1}=a, G_{2}=$ $a^{2}, G_{n+3}=a G_{n+2}-G_{n+1}+G_{n}, \forall n \geq 0$. Novamente usando o algoritmo "fominha"podemos mostrar que todo inteiro natural se escreve de forma única $n=\sum_{i=0}^{N} \varepsilon_{i} G_{i}$ onde a sequência $\left(\varepsilon_{i}\right)_{0 \leq i \leq N}$ satisfaz

$$
\varepsilon_{i} \varepsilon_{i-1} \varepsilon_{i-2} \varepsilon_{i-3}<_{l e x}(a-1)(a-1) 01, \forall i \geq 0
$$

onde colocamos $\varepsilon_{-1}=\varepsilon_{-2}=\varepsilon_{-3}=0$. Definimos o Fractal de Rauzy clássico por

$$
\mathcal{F}_{a}=\left\{\sum_{i=2}^{\infty} \varepsilon_{i} \alpha^{i} \mid \forall i \geq 2, \varepsilon_{i}=0,1, \ldots, a-1, \varepsilon_{i} \varepsilon_{i-1} \varepsilon_{i-2} \varepsilon_{i-3}<_{\text {lex }}(a-1)(a-1) 01\right\} .
$$

onde $\varepsilon_{-1}=\varepsilon_{-2}=\varepsilon_{-3}=0$.

O conjunto $\mathcal{F}_{a}$ será chamado de o fractal de Rauzy clássico e o conjunto $\mathcal{R}_{a}$ será chamado fractal de Rauzy com restrições associados ao polinômio $P_{a}$ (ver figuras abaixo, no caso a=2). O Fractal de Rauzy $\mathcal{R}_{a}$ com restrições foi definido por Messaoudi [17], mas até o momento tal fractal não havia sido muito estudado.

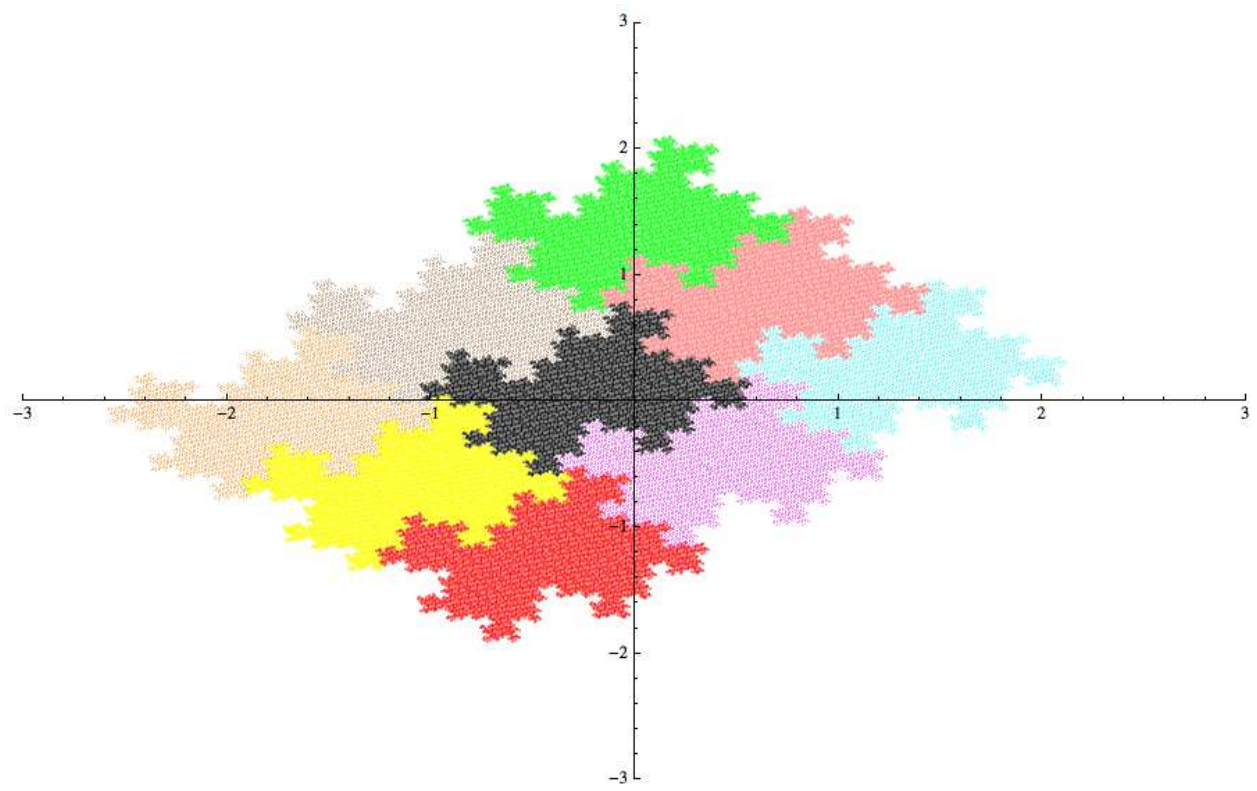

Figura 2: Fractal sem restrições com azulejamento

O estudo das fronteiras dos conjuntos $\mathcal{R}_{a}$ e $\mathcal{F}_{a}$ está intimamente relacionado com o estudo dos $\alpha$ desenvolvimentos, ou seja, a representação de números complexos na base $\alpha$. De fato, mostraremos que os pontos na fronteira de $\mathcal{R}_{a}$ e também de $\mathcal{F}_{a}$ são números complexos que têm mais de dois $\alpha$-desenvolvimentos com dígitos em $\{0,1, \ldots, a-1\}$. Construiremos um autômato finito que nos permitirá verificar quando dois $\alpha$-desenvolvimentos representam o mesmo número complexo. Fazendo 


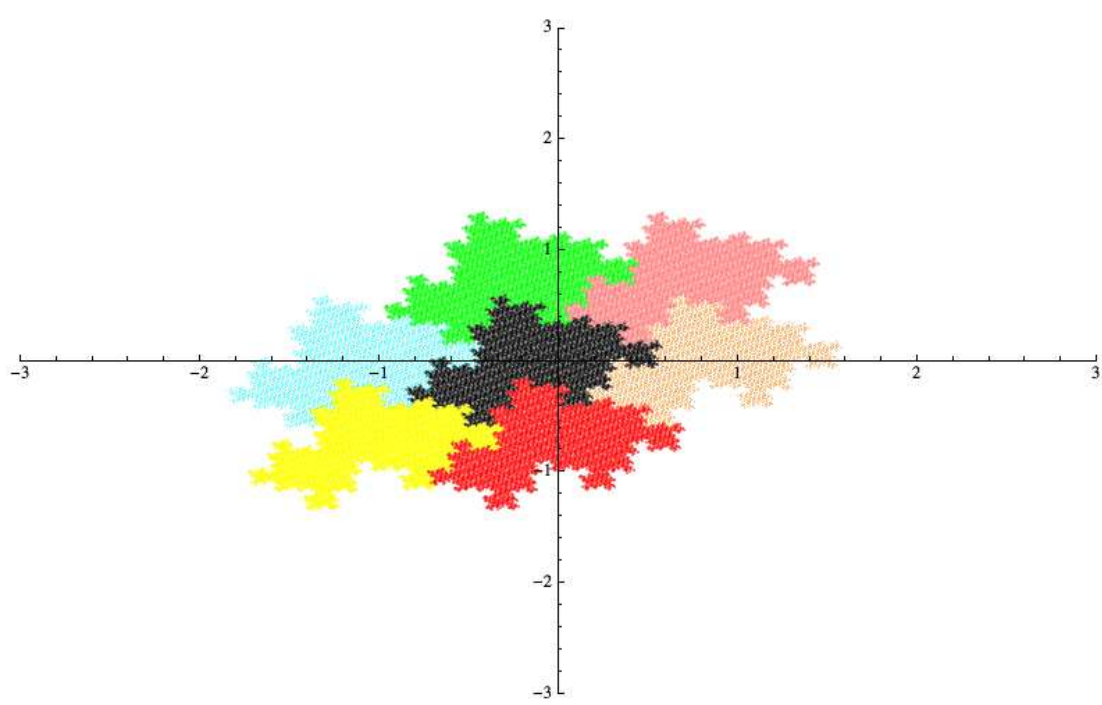

Figura 3: Fractal com restrições com azulejamento

uso desse autômato finito mostraremos que a fronteira de $\mathcal{R}_{a}$ e $\mathcal{F}_{a}$ são homeomorfas ao círculo e que tem a mesma dimensão de Hausdorff.

Nesta tese vamos provar várias propriedades de $\mathcal{R}_{a}$ e $\mathcal{F}_{a}$ e de suas fronteiras que resumimos nos seguintes teoremas:

Teorema 1: O conjunto $\mathcal{R}_{a}$ tem as seguintes propriedades:

1) $\mathcal{R}_{a}$ é um conjunto compacto;

2) $\mathcal{R}_{a}$ induz um azulejamento periódico do plano complexo, isto é,

$$
\mathbb{C}=\bigcup_{u \in \mathbb{Z}+\mathbb{Z} \alpha}\left(\mathcal{R}_{a}+u\right) \text { e } \operatorname{int}\left(\mathcal{R}_{a}+u\right) \cap\left(\mathcal{R}_{a}+v\right) \neq \emptyset, u, v \in \mathbb{Z}+\mathbb{Z} \alpha \text { implica que } u=v
$$

Teorema 2: A fronteira de $\mathcal{R}_{a}$ satisfaz as seguintes propriedades:

1)

$$
\partial \mathcal{R}_{a}=\bigcup_{u \in A} \mathcal{R}_{a} \cap\left(\mathcal{R}_{a}+u\right)
$$

onde $A$ é um conjunto finito contido em $\mathbb{Z}+\alpha \mathbb{Z}$ e $A \supset\{1, \alpha, \alpha-1,-1,-\alpha,-(\alpha-1)\}$.

2) Se $z \in \partial \mathcal{R}_{a}$ então existem $\left(\varepsilon_{i}\right)_{i \geq 2}$ e $\left(\varepsilon_{i}^{\prime}\right)_{i \geq l} \in \mathcal{N}, l<2$ tais que $z=\sum_{i=2}^{+\infty} \varepsilon_{i} \alpha^{i}=\sum_{i=l}^{+\infty} \varepsilon_{i}^{\prime} \alpha^{i}$ e $\varepsilon_{l}^{\prime} \neq 0$. onde $\mathcal{N}=\left\{\left(a_{i}\right)_{i \geq k}, k \in \mathbb{Z} \mid \forall n \geq k,\left(a_{i}\right)_{k \leq i \leq n}\right.$ é uma R-representação $\}$.

Teorema 3: Existe um autômato finito (grafo finito orientado com estados e flechas ligando estes estados) cujo conjunto dos estados é $S=\left\{0, \pm \alpha, \pm \alpha^{2}, \pm\left(\alpha-\alpha^{2}\right), \pm\left(1+(a-1) \alpha^{2}\right), \pm(1+(a-\right.$ 
$\left.\left.2) \alpha^{2}\right), \pm\left(1-\alpha+(a-1) \alpha^{2}\right), \pm\left(1-2 \alpha+a \alpha^{2}\right)\right\}$ tal que para todo $\left(a_{i}\right)_{i \geq l}$ e $\left(b_{i}\right)_{i \geq l} \in \mathcal{N}$, temos que $\sum_{i=l}^{\infty} a_{i} \alpha^{i}=\sum_{i=l}^{\infty} b_{i} \alpha^{i}$ se, e somente se, a sequência $\left(\left(a_{i}, b_{i}\right)\right)_{i \geq l}$ é um caminho infinito no autômato começando no estado inicial.

Como consequência desse Teorema obtemos o seguinte resultado:

\section{Teorema 4:}

1) Para todo $a \geq 2$ temos

$$
\partial \mathcal{R}_{a}=\bigcup_{u \in B} \mathcal{R}_{a} \cap\left(\mathcal{R}_{a}+u\right)
$$

onde $B=\{ \pm 1, \pm \alpha, \pm(\alpha-1)\}$.

2) Se $a=2$, então $\partial \mathcal{R}_{a}$ é homeomorfo ao círculo e a sua dimensão de Hausdorff é igual a $1,3593 \ldots$

Relacionado ao conjunto $\mathcal{F}_{a}$ temos o seguinte resultado:

Teorema 5: A fronteira de $\mathcal{F}_{a}$ é $\partial \mathcal{F}_{a}=\bigcup_{v \in B} \mathcal{F}_{a} \cap\left(\mathcal{F}_{a}+v\right)$ onde $B$ é um conjunto finito contido em $\mathbb{Z}\left(\alpha^{-3}+\alpha^{-1}\right)+\mathbb{Z}\left(1+\alpha^{-2}\right)$ cuja cardinalidade é par e maior ou igual a 8 e $\left\{ \pm\left(\alpha^{-3}+\right.\right.$ $\left.\left.\alpha^{-1}\right), \pm \alpha^{-1}, \pm\left(1+\alpha^{-2}\right), \pm\left(\alpha+\alpha^{-2}\right)\right\} \subset B$.

Fazendo uso do autômato, definimos uma parametrização da fronteira de $\mathcal{F}_{a}$, mostraremos que a fronteira é homeomorfa ao círculo e calcularemos sua dimensão de Hausdorff, a qual é igual ao do Fractal com restrições $\mathcal{R}_{a}$.

Esta tese está organizada da seguinte maneira: no Capítulo 1 enunciaremos alguns resultados sobre $\beta$-numeração. Esses resultados serão usados nos capítulos subsequentes. No Capítulo 2 provamos que o Fractal $\mathcal{R}_{a}$, é um conjunto compacto e que ele induz um azulejamento periódico do plano $\mathbb{C}$. Mostraremos que nesse azulejamento, $\mathcal{R}_{a}$ possui exatamente 6 vizinhos. No Capítulo 3 caracterizamos a fronteira de $\mathcal{R}_{a}$ usando o autômato. No Capítulo 4 utilizamos o autômato para parametrizar a fronteira de $\mathcal{R}_{a}$ no caso $a=2$, finalizando o capítulo com o cálculo da dimensão de Hausdorff da sua fronteira. Faremos a parametrização da fronteira de $\mathcal{F}_{a}$ no Capítulo 5 e também calcularemos a sua dimensão de Hausdorff. No Apêndice mostraremos alguns resultados complementares. 



\section{Capítulo}

\section{Sistemas de Numeração}

O objetivo deste capítulo é definir as $\beta$-representações $(\beta>1)$, isto é, escrever números reais na base $\beta$ com dígitos que são inteiros naturais usando o algoritmo "fominha". Com isso definimos um sistema de numeração sobre os inteiros, ou seja, definimos uma sequência de inteiros $\left(R_{n}\right)_{n \geq 0}$ tal que todo inteiro natural $N$ se escreve da seguinte maneira $N=\sum_{n=0}^{k(n)} \varepsilon_{n} R_{n}$ onde $\left(\varepsilon_{n}\right)_{0 \leq n \leq k(n)}$ é uma $\beta$-representação. Enunciaremos alguns resultados sobre as $\beta$-representações no caso onde $\beta$ é um número de Pisot cúbico. A relação com o fractal de Rauzy vem do fato que esse último é definido como os conjuntos das somas $\sum_{i=2}^{+\infty} \varepsilon_{i} \alpha^{i}$ onde $\left(\varepsilon_{i}\right)_{i \geq 2}$ é uma $\beta$-representação onde $\beta>1$ é um número de Pisot.

\section{$1.1 \beta$-Numeração}

Seja $\beta>1$ um número real. Uma representação de um número real não negativo $x$ na base $\beta$ é uma sequência infinita $\left(x_{i}\right)_{i \leq k}, k \in \mathbb{N}, x_{i} \in \mathbb{Z}_{+}$tal que

$$
x=x_{k} \beta^{k}+x_{k-1} \beta^{k-1}+\ldots+x_{1} \beta+x_{0}+x_{-1} \beta^{-1}+x_{-2} \beta^{-2}+\ldots
$$

Esse número é representado pela sequência $x=x_{k} x_{k-1} \ldots x_{1} x_{0} x_{-1} x_{-2} \ldots$

Uma $\beta$-representação, chamada $\beta$-expansão pode ser obtida pelo algoritmo conhecido como "fominha"ou greedy. Denotamos por $\lfloor x\rfloor$ e $\{x\}$ a parte inteira e a parte fracionária do número $x$, respectivamente. Existe um número $k \in \mathbb{Z}$ tal que $\beta^{k} \leq x<\beta^{k+1}$. Seja $x_{k}=\left\lfloor\frac{x}{\beta^{k}}\right\rfloor$ e $r_{k}=\left\{\frac{x}{\beta^{k}}\right\}$. Então para $i<k$, seja $x_{i}=\left\lfloor\beta r_{i+1}\right\rfloor$ uma fórmula de recorrência e $r_{i}=\left\{\beta r_{i+1}\right\}$. Temos então

$$
x=x_{k} \beta^{k}+x_{k-1} \beta^{k-1}+\ldots
$$

se $k<0$ (isto é, se $x<1$ ), colocamos $x_{0}=x_{-1}=\ldots=x_{k+1}=0$. 
Se uma $\beta$-representação termina com uma infinidade de zeros, omitimos esses zeros e dizemos que ela é finita. Se $\beta$ é inteiro, os dígitos $x_{i}$ pertencem ao conjunto $A=\{0, \ldots, \beta-1\}$ caso contrário pertencem ao conjunto $A=\{0, \ldots,\lfloor\beta\rfloor\}$.

O ponto entre a parte inteira e a fracionária da $\beta$-representação algumas vezes será omitido, assim a sequência infinita poderá ser considerada um elemento de $A^{\mathbb{N}}$.

Para os números $0 \leq x<1$, a expansão definida acima coincide com a $\beta$-representação de Rényi, a qual pode ser definida da seguinte maneira: $\operatorname{sejam} \beta>1$ um número real e $T:[0,1] \longrightarrow[0,1]$ uma transformação dada por $T_{\beta}(y)=\{\beta y\}$, onde \{\} designa a parte fracionária. Para $x \in[0,1)$ temos $x_{-j}=\left\lfloor\beta T_{\beta}^{j-1}(x)\right\rfloor$ para $j=1,2$. Todo número real $x \geq 0$ pode ter o seguinte desenvolvimento: $x=\sum_{n=0}^{\infty} x_{n} \beta^{-n}$ onde $x_{n}$ é definido da seguinte maneira:

Se $x>1$ então $x_{0}=\lfloor x\rfloor$ e $x_{n+1}=\left\lfloor\beta T^{n}(\{x\})\right\rfloor, n \in \mathbb{N}$ e se $0 \leq x \leq 1$ então $x_{0}=0$ e $x_{n+1}=\left\lfloor\beta T^{n}(x)\right\rfloor, n \in \mathbb{N}$.

Para $x=1$ os dois algoritmos ficam diferentes. A $\beta$-expansão de 1 é $1=1.000 \ldots$, enquanto a $\beta$-representação de Rényi de 1 é $d(x, \beta)=. t_{-1} t_{-2} \ldots$, onde $t_{-j}=\left\lfloor\beta T_{\beta}^{j-1}(1)\right\rfloor, \forall j \geq 1$.

Seja Fin $(\beta)$ o conjunto dos números reais não negativos que possuem uma $\beta$-expansão finita. Algumas vezes denotamos uma $\beta$-expansão finita $x_{n} \ldots x_{k}, k \leq n$, por $\left(x_{i}\right)_{n \geq i \geq k}$. Denotamos o conjunto de $\beta$-expansões finitas por $F_{\beta}$. Chamamos

$$
E_{\beta}=\left\{\left(x_{i}\right)_{i \geq k}, k \in \mathbb{Z} \mid \forall n \geq k,\left(x_{i}\right)_{n \geq i \geq k} \in F_{\beta}\right\}
$$

Dizemos que $\beta$ tem a propriedade de Finitude ou que $\beta$ satisfaz a propriedade $(F)$ se

$$
\mathbb{Z}[\beta] \cap[0,+\infty[\subset \operatorname{Fin}(\beta) .
$$

onde $\mathbb{Z}[\beta]$ é o anel gerado por $\mathbb{Z}$ e $\beta$. Se $\beta$ satisfaz a propriedade $(F)$, então $d(1, \beta)$ é finita, pois $\beta-\lfloor\beta\rfloor \in \operatorname{Fin}(\beta)$.

Definição 1.1.1 Dizemos que $\beta$ é um número de Pisot se $\beta$ é um número inteiro algébrico com conjugados em relação a Galois com módulo menor do que 1. Além disso, se

$$
X^{d}+b_{d-1} X^{d-1}+\ldots+b_{0}
$$

é polinômio minimal de $\beta$ então $\beta$ é dito unidade de Pisot se $b_{0}= \pm 1$.

Seja $N \in \mathbb{Z}$, denotamos por $\operatorname{Fin}_{N}(\beta)$ o conjunto dos números reais positivos $x$ cuja $\beta$-representação satisfaz, $x_{k}=0$ para todo $k<N$. Algumas vezes denotaremos uma $\beta$-representação $x_{n} \ldots x_{k}, n \geq k$ 
por $\left(x_{i}\right)_{n \geq i \geq k}$. Chamamos

$$
E_{\beta}=\left\{\left(x_{i}\right)_{i \geq k}, k \in \mathbb{Z} \mid \forall n \geq k,\left(x_{i}\right)_{n \geq i \geq k} \text { é uma } \beta \text {-representação finita }\right\} .
$$

Dizemos que $\beta$ satisfaz a propriedade $(F)$ se

$$
\mathbb{Z}[\beta] \cap[0,+\infty[\subset \operatorname{Fin}(\beta) .
$$

Sabe-se que a classe dos números de Pisot que possuem a propriedade $(F)$ é igual à classe dos números reais que são exatamente as raízes dominantes do seguinte polinômio (com coeficientes inteiros):

$$
x^{3}-a x^{2}-b x-1, a \geq 0 \quad e \quad-1 \geq b \geq a+1 .
$$

(se $b=-1$ adicionamos a restrição $a \geq 2$ ).

A $\beta$-representação de Rényi de 1 em relação a esses números é dada pela proposição seguinte:

Proposição 1.1.2 [1] Seja $\beta$ um número de Pisot cúbico unitário e que satisfaz a propriedade (F) com polinômio minimal $x^{3}-a x^{2}-b x-1$. Então uma das seguintes possibilidades abaixo ocorre:

$$
\begin{array}{ccc}
\text { Se } 0 \geq b \geq a, \text { então } d(1, \beta) & = & \cdot a b 1, \\
\text { Se } b=-1 \text { e } a \geq 2, \text { ent } \tilde{a} o \quad d(1, \beta) & = & \cdot(a-1)(a-1) 01, \\
\text { Se } b=a+1, \text { então } d(1, \beta) & = & \cdot(a+1) 00 a 1 .
\end{array}
$$

Observação 1.1.3 Por exemplo, no caso $b=-1$ e $a \geq 2$ (caso de interesse deste trabalho) temos

$$
1=\frac{a-1}{\beta}+\frac{a-1}{\beta^{2}}+\frac{1}{\beta^{4}}
$$

Definição 1.1.4 [12]Dizemos que uma sequência $\left(a_{n}\right)_{k \leq n \leq p}$ é superior anti-lexicograficamente à sequência $\left(b_{n}\right)_{k^{\prime} \leq n \leq p^{\prime}}$ se existe um inteiro $s \in \mathbb{Z}$ tal que para todo $n>s, a_{n}=b_{n}$ e $a_{s}>b_{s}$.

Proposição 1.1.5 [23] Seja $x_{n} \ldots x_{0}$ e $y_{m} \ldots y_{0}$ duas $\beta$-representações. Então $\sum_{i=0}^{n} x_{i} \beta^{i}<_{\text {lex }}$ $\sum_{i=0}^{m} y_{i} \beta^{i}$ se, e somente se, $x_{n} \ldots x_{0}<_{l e x} y_{m} \ldots y_{0}$

Lembrando que $\beta$ é um número de Pisot unitário se também é unidade do anel de inteiros de $\mathbb{Q}[\beta]$. E vamos considerar que $\beta$ satisfaz a propriedade $(F)$ e denotamos por $\alpha, \gamma$ seus conjugados em relação a Galois. Seja $P(x)=x^{3}-a x^{2}-b x-1$ o polinômio minimal de $\beta$. 


\subsection{Sistema de Numeração sobre os inteiros}

Nessa seção vamos assumir que $\beta$ satisfaz as condições da Proposição (1.1.2) e que $x^{3}-a x^{2}-$ $b x-1$ é o polinômio minimal de $\beta$.

Um sistema de numeração sobre os inteiros induzido pelas $\beta$-representações é dado da seguinte maneira: supomos que $d(1, \beta)=\cdot a_{-1} \ldots a_{-t}$ onde $a_{-t} \neq 0$. Seja $\left(R_{n}\right)_{n \geq 0}$ a sequência de números inteiros definida por: $R_{0}=1, R_{1}=a, R_{2}=a^{2}+b, R_{n+3}=a R_{n+2}+b R_{n+1}+R_{n}, \forall n \geq 0$.

Como o polinômio $Q(x)=x^{t}-a_{-1} x^{t-1}-\ldots-a_{-t}$ é múltiplo do polinômio $x^{3}-a x^{2}-b x-1$, a sequência $\left(R_{n}\right)_{n \geq 0}$ satisfaz (provando-se por indução)

$$
R_{n+t}=a_{-1} R_{n+t-1}+a_{-2} R_{n+t-2}+\ldots+a_{-t} R_{n}, \forall n \geq 0 .
$$

Proposição 1.2.1 [21] Todo número inteiro $n$ tem uma única R-representação $\left(\varepsilon_{j}\right)_{k(n) \geq j \geq 0}$ tal que $n=\sum_{j=0}^{k(n)} \varepsilon_{j} R_{j}$ com $\varepsilon_{j} \geq 0$. Em particular:

a) Se $a \geq b \geq 0$ e $a \neq 0$ então para todo $i \geq 2$, a palavra $\varepsilon_{i} \varepsilon_{i-1} \varepsilon_{i-2}<_{\text {lex }}$ ab1, $\varepsilon_{1} \varepsilon_{0}<_{\text {lex }}$ $a b, \varepsilon_{0}<_{l e x} a$,

b) Se $b=-1$ e $a \geq 2$ então para todo $i \geq 3, \varepsilon_{i} \varepsilon_{i-1} \varepsilon_{i-2} \varepsilon_{i-3}<_{l e x}(a-1)(a-1) 01, \varepsilon_{2} \varepsilon_{1} \varepsilon_{0}<_{l e x}$ $(a-1)(a-1) 0, \varepsilon_{1} \varepsilon_{0}<_{l e x}(a-1)(a-1), \varepsilon_{0}<_{l e x} a$,

c) $S e b=a+1$ e $a \geq 1$ então para todo $i \geq 4$,

$\varepsilon_{i} \varepsilon_{i-1} \varepsilon_{i-2} \varepsilon_{i-3} \varepsilon_{i-4}<_{l e x}(a+1) 00 a 1, \varepsilon_{3} \varepsilon_{2} \varepsilon_{1} \varepsilon_{0}<_{l e x}(a+1) 00 a, \varepsilon_{2} \varepsilon_{1} \varepsilon_{0}<_{l e x}(a+1) 00, \varepsilon_{1} \varepsilon_{0}<_{l e x}$ $(a+1) 1, \varepsilon_{0}<a$,

d) Se $a=0$ e $b=1$ então para todo $i \geq 4, \varepsilon_{i} \varepsilon_{i-1} \varepsilon_{i-2} \varepsilon_{i-3} \varepsilon_{i-4}<_{l e x} 10001$ e $\varepsilon_{0}=\varepsilon_{1}=\varepsilon_{2}=\varepsilon_{3}=$ $\varepsilon_{4}=\varepsilon_{5}=0$.

Demonstração: Os dígitos $\left(\varepsilon_{j}\right)_{k(n) \geq j \geq 0}$ são obtidos aplicando o algoritmo "greedy"para inteiros.

a) Se $a \geq b \geq 0$ e $a \neq 0$, então $\left(R_{n}\right)_{n \geq 0}$ é uma sequência crescente de inteiros naturais. Logo, pela definição do algoritmo "greedy", prova-se que para todo $0 \leq j \leq k(n), \sum_{i=0}^{j} \varepsilon_{i} R_{i}<R_{j+1}$. Portanto,

$$
\varepsilon_{j} \varepsilon_{j-1} \ldots \varepsilon_{j-t+1}<_{l e x} a_{-1} a_{-2} \ldots a_{-t}, \forall j \geq t-1
$$


Pela relação (1.1) e a Proposição, temos $\varepsilon_{i} \varepsilon_{i-1} \varepsilon_{i-2}<_{\text {lex }}$ ab1. Como $n-\sum_{i=1}^{k(n)} \varepsilon_{i} R_{i}=\varepsilon_{0} R_{0}=$ $\varepsilon_{0}<R_{1}=a$ e $n-\sum_{i=2}^{k(n)} \varepsilon_{1} R_{1}+\varepsilon_{0} R_{0}<R_{2}=a^{2}+b=a R_{1}+b R_{0}$, obtemos $\varepsilon_{0}<a e$ $\varepsilon_{1} \varepsilon_{0}<_{\text {lex }} a b$.

b) Se $b=-1$ e $a \geq 2$, então usando o mesmo argumento do caso 1, obtemos que para todo $i \geq 3, \varepsilon_{i} \varepsilon_{i-1} \varepsilon_{i-2} \varepsilon_{i-3}<_{l e x}(a-1)(a-1) 01$. As outras condiçôes são obtidas, pois $\varepsilon_{0} R_{0}<R_{1}=$ $a, \varepsilon_{1} R_{1}+\varepsilon_{0} R_{0}<R_{2}=(a-1) R_{1}+(a-1) R_{0}$ e $\varepsilon_{2} R_{2}+\varepsilon_{1} R_{1}+\varepsilon_{0} R_{0}<R_{3}=(a-1) R_{2}+(a-1) R_{1}$.

c) Se $b=a+1$ e $a \geq 1$ então pelo mesmo argumento do caso (a) temos para todo $i \geq$ $4, \varepsilon_{i} \varepsilon_{i-1} \varepsilon_{i-2} \varepsilon_{i-3} \varepsilon_{i-4}<_{l e x}(a+1) 00 a 1$. As outras condições são obtidas pois $R_{1}=a R_{0}, R_{2}=$ $(a+1) R_{1}+R_{0}, R_{3}=(a+1) R_{2}$ e $R_{4}=(a+1) R_{3}+a R_{0}$.

d) Se $a=0$ e $b=1$ então a relação (1.1) e a proposição implicam que para todo $i \geq 4$, $\varepsilon_{i} \varepsilon_{i-1} \varepsilon_{i-2} \varepsilon_{i-3} \varepsilon_{i-4}<_{\text {lex }}$ 10001. Por outro lado, temos $R_{0}=R_{2}=R_{3}=R_{4}=1, R_{1}=0, R_{5}=$ $R_{6}=2$. Então temos $\varepsilon_{0}=\varepsilon_{1}=\varepsilon_{2}=\varepsilon_{3}=\varepsilon_{5}=0$. 



\section{Capítulo}

\section{Propriedades do Fractal de Rauzy}

Neste Capítulo provamos que o Fractal de Rauzy $\mathcal{R}$ é um conjunto compacto que induz um azulejamento periódico do plano.

\subsection{Propriedades do Fractal de Rauzy}

O Fractal de Rauzy $\mathcal{F}$ (sem restriçôes) foi estudado por vários matemáticos, ver por exemplo [26], [18] e [29].

$O$ conjunto $\mathcal{F}$ possui várias propriedades, em particular, ele é um conjunto compacto, conexo ([26] ), com interior simplesmente conexo [18] e além disso $0 \in \operatorname{int}(\mathcal{F})$ [1].

Em relação ao conjunto $\mathcal{R}$ (fractal de Rauzy com restriçôes), nada é conhecido na literatura. Neste trabalho propomos provar alguns resultados. Antes disso daremos algumas notaçôes.

Notações: Seja $\mathcal{N}=\left\{\left(a_{i}\right)_{i \geq k}, k \in \mathbb{Z} \mid \forall n \geq k,\left(a_{i}\right)_{k \leq i \leq n}\right.$ é uma R-representação $\}$, isto é, para todo $n \geq k+3, a_{n} a_{n-1} a_{n-2} a_{n-3}<_{\text {lex }}(a-1)(a-1) 01, a_{k}<_{\text {lex }}(a-1), a_{k+1} a_{k}<_{\text {lex }}(a-1)(a-1)$ e $a_{k+2} a_{k+1} a_{k}<_{\text {lex }}(a-1)(a-1) 0$.

Denotamos por $\mathcal{N}_{f}=\left\{\left(a_{i}\right)_{k \leq i \leq n}, k, n \in \mathbb{Z},\left(a_{i}\right)_{k \leq i \leq n}\right.$ é uma R-representação $\}$.

Seja $z \in \mathbb{C}$ e $A \subset \mathbb{C}$, temos $A+z=\{x+z \mid x \in A\}$ e $z A=\{z x \mid x \in A\}$. Notamos por int $(A) o$ conjunto dos pontos interiores de $A$ e $\bar{A}$ o fecho de $A$.

Teorema 2.1.1 O conjunto $\mathcal{R}$ satisfaz as seguintes propriedades:

1. O conjunto $\mathcal{R}$ é compacto;

2. O conjunto $\mathcal{R}$ induz um azulejamento periódico do plano $\mathbb{C}$, isto é, $\mathbb{C}=\bigcup_{p, q \in \mathbb{Z}}(\mathcal{R}+p+q \alpha)$ e para todo $p, q, r, s \in \mathbb{Z}$, int $(\mathcal{R}+p+q \alpha) \cap(\mathcal{R}+r+s \alpha) \neq \emptyset$ implica que $p=r$ e $q=s$.

Vamos fazer a demonstração fragmentando o resultado em algumas proposiçôes: 
Proposição 2.1.2 O conjunto $\mathcal{R}$ é compacto.

\section{Demonstração:}

Seja $\left(z_{n}\right)_{n \geq 0}$ uma sequência de elementos de $\mathcal{R}$ tal que $\lim _{n \longrightarrow+\infty} z_{n}=z$. Vamos mostrar que $z \in \mathcal{R}$. Supomos que $z_{n}=\sum_{i=2}^{\infty} \varepsilon_{i}^{(n)} \alpha^{i}$, onde $\left(\varepsilon_{i}^{(n)}\right)_{i \geq 2} \in \mathcal{M}$.

Seja $a \in \mathcal{A}=\{0,1, \ldots, a-1\}$.

Considere $\mathcal{C}_{a}=\left\{k \in \mathbb{N}, \varepsilon_{2}^{(k)}=a\right\}$. Temos que $\mathbb{N}=\bigcup_{a \in \mathcal{A}} \mathcal{C}_{a}$, como $\mathcal{A}$ é finito existe um $a_{2} \in \mathcal{A}$, tal que $\mathcal{C}_{a_{2}}$ é infinito. Seja $k_{2} \in \mathcal{C}_{a_{2}}$. Considere $\mathcal{C}_{a_{2} b}=\left\{k \in \mathcal{C}_{a_{2}}, \varepsilon_{3}^{(k)}=b\right\}$ onde $b \in \mathcal{A}$.

Da mesma forma existe $a_{3} \in \mathcal{A}$ tal que

$$
\mathcal{C}_{a_{2} a_{3}}=\left\{k \in \mathbb{N}, \varepsilon_{2}^{(k)}=a_{2}, \varepsilon_{3}^{(k)}=a_{3}\right\} \text { é infinito. }
$$

Seja $k_{3} \in \mathcal{C}_{a_{2} a_{3}}$, temos $\varepsilon_{2}^{\left(k_{3}\right)}=a_{2}$ e $\varepsilon_{3}^{\left(k_{3}\right)}=a_{3}$.

Continuando com o mesmo processo (diagonal de Cantor) construimos uma sequência $\left(a_{i}\right)_{i \geq 2} e$ $\left(k_{n}\right)_{n \geq 2}$ tal que

$$
\varepsilon_{2}^{\left(k_{n}\right)}=a_{2}, \varepsilon_{3}^{\left(k_{n}\right)}=a_{3}, \ldots, \varepsilon_{n}^{\left(k_{n}\right)}=a_{n} \text { para todo } n \in \mathbb{N}
$$

Considere $z_{k_{n}}=\sum_{i=2}^{+\infty} \varepsilon_{i}^{\left(k_{n}\right)} \alpha^{i}$ temos que $z_{k_{n}} \longrightarrow z=\sum_{i=2}^{+\infty} a_{i} \alpha^{i}$, por (2.1) e $z \in \mathcal{R}$. Por outro lado $\mathcal{R}$ é limitado pois se $z=\sum_{i=2}^{\infty} \varepsilon_{i} \alpha^{i} \in \mathcal{R}$ então

$$
|z| \leq \sum_{i=2}^{\infty}|a-1||\alpha|^{i}=\frac{(a-1)|\alpha|^{2}}{1-|\alpha|} .
$$

Portanto, $\mathcal{R}$ é compacto.

Proposição 2.1.3 $\mathcal{R}$ induz um azulejamento periódico em $\mathbb{C}$.

Para provar essa proposição precisamos dos seguintes resultados.

Antes disso considere a sequência $\left(F_{n}\right)$ definida por $F_{0}=0, F_{1}=0, F_{2}=1, F_{n+3}=a F_{n+2}-$ $F_{n+1}+F_{n}, n \geq 0$. Observe que $F_{n+2}=R_{n}, \forall n \geq 0$ (ver subseção 1.2 do Capítulo 1).

Lema 2.1.4 As propriedades seguintes são válidas (ver Apêndice):

i) Todo inteiro natural $n$ pode ser escrito de uma única forma: $n=\sum_{i=2}^{N} \varepsilon_{i} F_{i}$ onde $\left(\varepsilon_{i}\right)_{2 \leq i \leq N} \in$ $\mathcal{N}_{f}$.

ii) Sejam $\left(a_{i}\right)_{l \leq i \leq N} \in \mathcal{N}_{f}$ e $\left(b_{i}\right)_{l^{\prime} \leq i \leq \infty} \in \mathcal{N}$ tais que $a_{l}>0$ e $b_{l^{\prime}}>0$. Se $\sum_{i=l}^{N} a_{i} \alpha^{i}=\sum_{i=l^{\prime}}^{\infty} b_{i} \alpha^{i}$ então $l=l^{\prime}$ e para todo $i \geq N, b_{i}=0$ e para todo $l \leq i \leq N, a_{i}=b_{i}$. 
iii) Seja $\left(\varepsilon_{i}\right)_{2 \leq i \leq N} \in \mathcal{N}_{f}$ então $\sum_{i=2}^{N} \varepsilon_{i} \alpha^{i} \in \operatorname{int}(\mathcal{R})$. Em particular, $0 \in \operatorname{int}(\mathcal{R})$.

iv) Seja $z \in \mathbb{Z}[\beta] \cap \mathbb{R}^{+}$então existe $\left(a_{i}\right)_{k \leq i \leq l} \in \mathcal{N}_{f}, k \leq l$ tal que $z=\sum_{i=k}^{l} a_{i} \beta^{i}$.

v) Para todo $n \geq 2$ temos que $\beta^{n}=F_{n} \beta^{2}+\left(F_{n-2}-F_{n-1}\right) \beta+F_{n-1}$, em particular se $\left(\varepsilon_{i}\right)_{2 \leq i \leq l} \in \mathcal{N}_{f}$ então $\sum_{i=2}^{l} \varepsilon_{i} \beta^{i}=n \beta^{2}+r(n) \beta+s(n)$ onde $n=\sum_{i=2}^{l} \varepsilon_{i} F_{i}, r(n)=\sum_{i=2}^{l} \varepsilon_{i}\left(F_{i-2}-F_{i-1}\right) e$ $s(n)=\sum_{i=2}^{l} \varepsilon_{i} F_{i-1}$.

vi) Sejam $\left(a_{i}\right)_{l \leq i \leq k}$ e $\left(b_{i}\right)_{l \leq i \leq k}$ pertencentes a $\mathcal{N}_{f}$ então $\sum_{i=l}^{k} a_{i} \beta^{i}<\sum_{i=l}^{k} b_{i} \beta^{i}$ se, e somente se, $\left(a_{i}\right)_{l \leq i \leq k}<_{l e x}\left(b_{i}\right)_{l \leq i \leq k}$.

vii) Sejam $c, d \in \mathbb{R}$ tais que $\alpha^{2}=c+d \alpha$, então 1, c e d são $\mathbb{Q}$-Linearmente Independentes.

Proposição 2.1.5 $\mathbb{C}=\bigcup_{p, q \in \mathbb{Z}}(\mathcal{R}+p+q \alpha)$.

\section{Demonstração da Proposição 2.1.5:}

Seja $z \in \mathbb{C}$, vamos provar que existem $m, n \in \mathbb{Z}$ tais que $z+m+n \alpha \in \mathcal{R}$.

Seja $\epsilon>0$, pelo item (vii) do Lema (2.1.4) e pelo Teorema de Kronecker, o conjunto $\left\{n \alpha^{2}+\right.$ $p \alpha+q, n \in \mathbb{N}, p, q \in \mathbb{Z}\}$ é denso em $\mathbb{C}$. Logo existe uma sequência $\left(z_{k}\right)_{k \geq 0} \in \mathbb{C}$ tal que

$$
z_{k}=n_{k} \alpha^{2}+p_{k} \alpha+r_{k}, n_{k} \in \mathbb{N}, p_{k}, q_{k} \in \mathbb{Z}
$$

existe $k_{0}$ tal que para todo $k \geq k_{0},\left|z_{k}-z\right|<\epsilon$. Seja $A_{k}=n_{k} \alpha^{2}+r\left(n_{k}\right) \alpha+s\left(n_{k}\right)$, onde $r\left(n_{k}\right)$ e $s\left(n_{k}\right)$ são definidos no item (v) do Lema (2.1.4). Temos $A_{k} \in \mathcal{R}$.

Por outro lado,

$$
\begin{array}{ccc}
A_{k} & =n_{k} \alpha^{2}+p_{k} \alpha+r_{k}+\left(r\left(n_{k}\right)-p_{k}\right) \alpha+\left(s\left(n_{k}\right)-r_{k}\right) \\
& = & z_{k}+t_{k} \alpha+m_{k}, \text { onde }
\end{array}
$$

$t_{k}=r\left(n_{k}\right)-p_{k}$ e $m_{k}=s\left(n_{k}\right)-r_{k}$.Logo, $z_{k}+t_{k} \alpha+m_{k} \in \mathcal{R}$.

Sendo $\mathcal{R}$ compacto temos para todo $k \geq k_{0}$,

$$
\left|z+t_{k} \alpha+m_{k}\right| \leq\left|z-z_{k}\right|+\left|z_{k}+t_{k} \alpha+m_{k}\right|<\epsilon+d
$$

onde $d=\min \{|z-p|, \quad z \in \mathcal{R}, p \in \mathbb{Z}+\mathbb{Z} \alpha\}$.

Assim para todo $k \geq k_{0},-t_{k} \alpha-m_{k} \in B(z, \epsilon+d)$.

Por outro lado, como $\mathbb{Z}+\mathbb{Z} \alpha$ é um reticulado então $B(z, \epsilon+d)$ contém somente um número finito de $t_{k} \alpha+m_{k}$, logo existe uma sequência crescente $\left(k_{i}\right)_{i \geq 1}$ de números inteiros tal que para todo $i, j \in \mathbb{N}, t_{k_{i}} \alpha+r_{k_{i}}=t_{k_{j}} \alpha+r_{k_{j}}$. Logo existem $t, r \in \mathbb{Z}$ tais que $t_{k_{i}}=t_{k_{j}}=t$ e $r_{k_{i}}=r_{k_{j}}=r$, para todo $i, j \in \mathbb{N}$. Como $z_{k_{i}}+t_{k_{i}} \alpha+m_{k_{i}} \in \mathcal{R}, z_{k_{i}} \longrightarrow z$ e $\mathcal{R}$ é fechado, temos que $z+t \alpha+r \in \mathcal{R}$. 
Proposição 2.1.6 Para quaisquer $u, v \in \mathbb{Z}+\mathbb{Z} \alpha$ temos $\operatorname{int}(\mathcal{R}+u) \cap(\mathcal{R}+v) \neq \emptyset$ implica que $u=v$

Demonstração da Proposição 2.1.6: Basta provar que se $(\operatorname{int}(\mathcal{R})+u) \cap \mathcal{R} \neq \emptyset, u \in \mathbb{Z}+\mathbb{Z} \alpha$ ent $\tilde{a} O \quad u=0$.

Vamos fazer a prova por absurdo. Assuma que existam inteiros $p, q \in \mathbb{Z}$ e um elemento $z=$ $\sum_{i=2}^{\infty} \varepsilon_{i} \alpha^{i} \in \mathcal{R}$ tal que $z+p+q \alpha \in \operatorname{int}(\mathcal{R})$. Logo existe um inteiro $n_{0} \geq 0$ tal que para todo $n \geq n_{0}$

$$
\sum_{i=2}^{n} \varepsilon_{i} \alpha^{i}+p+q \alpha \in \mathcal{R}
$$

Caso 1: O conjunto $\left\{i \geq 2, \varepsilon_{i} \neq 0\right\}$ é infinito.

Neste caso, como $\beta>1$, então existe um inteiro $N \geq n_{0}$ tal que $\sum_{i=2}^{N} \varepsilon_{i} \beta^{i}+p+q \beta>0$. Pelo item (iv) do Lema 2.1.4 deduzimos que

$$
\sum_{i=2}^{N} \varepsilon_{i} \beta^{i}+p+q \beta=\sum_{i=l}^{M} d_{i} \beta^{i}, \text { onde }\left(d_{i}\right)_{l \leq i \leq M} \in \mathcal{N}_{f}, l, M \in \mathbb{Z} .
$$

A partir de (2.2) e (2.3) temos que $\sum_{i=l}^{M} d_{i} \alpha^{i}=\sum_{i=2}^{\infty} e_{i} \alpha^{i} \in \mathcal{R}$.

Assim, do item (ii) do Lema 2.1.4 temos que $e_{i}=0$ para todo $i>M$. Logo,

$$
\begin{aligned}
\sum_{i=2}^{N} \varepsilon_{i} \beta^{i}+p+q \beta & =\sum_{i=2}^{M} e_{i} \beta^{i} \\
& =\sum_{i=l}^{M} d_{i} \beta^{i} .
\end{aligned}
$$

Do item (iv) do Lema 2.1.4 temos que

$$
\widetilde{n} \beta^{2}+(r(\widetilde{n})+q) \beta+(s(\widetilde{n})+p)=\widetilde{l} \beta^{2}+r(\widetilde{l}) \beta+s(\widetilde{l}) .
$$

onde $\widetilde{n}=\sum_{i=2}^{N} \varepsilon_{i} F_{i}$ e $l=\sum_{i=2}^{M} e_{i} F_{i}$. Assim, $\widetilde{l}=\widetilde{n}$ e $\varepsilon_{i}=e_{i}$ para todo $i$ (devido a unicidade na base $\left.F_{i}\right)$. Portanto, $p=q=0$.

Caso 2: O conjunto $\left\{i \geq 2, \varepsilon_{i} \neq 0\right\}$ é finito.

Seja $N=\max \left\{i \geq 2, \varepsilon_{i} \neq 0\right\}$. Se $\sum_{i=2}^{N} \varepsilon_{i} \beta^{i}+p+q \beta \geq 0$ então usa-se o mesmo argumento que no Caso 1.

Vamos assumir agora que $\sum_{i=2}^{N} \varepsilon_{i} \beta^{i}+p+q \beta<0$. Temos que

$$
\sum_{i=2}^{N} \varepsilon_{i} \alpha^{i}+p+q \alpha=\sum_{i=2}^{\infty} d_{i} \alpha^{i}, \text { onde }\left(d_{i}\right)_{i \geq 2} \in \mathcal{N}
$$

Como $\sum_{i=2}^{N} \varepsilon_{i} \alpha^{i}$ é um ponto interior de $\mathcal{R}$ (item(iii) do Lema(2.1.4)) então existe um inteiro 
não negativo $M$ tal que

$$
-p-q \alpha+\sum_{i=2}^{M} d_{i} \alpha^{i}=\sum_{i=2}^{\infty} e_{i} \alpha^{i} \in \mathcal{R} .
$$

Como $-p-q \beta+\sum_{i=2}^{M} d_{i} \beta^{i}>0$ então $-p-q \beta+\sum_{i=2}^{M} d_{i} \beta^{i}=\sum_{i=l}^{K} f_{i} \beta^{i}$ onde $\left(f_{i}\right)_{l \leq i \leq K} \in \mathcal{N}_{f}$. Logo,

$$
-p-q \alpha+\sum_{i=2}^{M} d_{i} \alpha^{i}=\sum_{i=l}^{K} f_{i} \alpha^{i}=\sum_{i=2}^{\infty} e_{i} \alpha^{i}
$$

onde $\left(f_{i}\right)_{l \leq i \leq K} \in \mathcal{N}_{f}$ e $l, K \in \mathbb{Z}$.

Pela Proposição 2.1.4 item(ii) deduzimos que $e_{i}=0$ para todo $i>K$ e pelo mesmo argumento usado no Caso 1 temos que $p=q=0$.

Observação 2.1.7 Na prova anterior, usamos o fato que se $\sum_{i=0}^{n} a_{i} \beta^{i}=\sum_{i=0}^{n} b_{i} \beta^{i}, a_{i}, b_{i} \in \mathbb{Z}$ então $\sum_{i=0}^{n} a_{i} \alpha^{i}=\sum_{i=0}^{n} b_{i} \alpha^{i}$. Esse resultado vem do fato que $\beta$ e $\alpha$ são algebricamente conjugados.

Teorema 2.1.8 A fronteira de $\mathcal{R}$ satisfaz as seguintes propriedades:

1)

$$
\partial \mathcal{R}=\bigcup_{u \in A} \mathcal{R} \cap(\mathcal{R}+u)
$$

onde $A$ é um conjunto finito contido em $\mathbb{Z}+\alpha \mathbb{Z}$, cuja cardinalidade é par e maior ou igual a $6 e\{1, \alpha, \alpha-1,-1,-\alpha,-(\alpha-1)\} \subset A$.

2) Seja $z \in \partial \mathcal{R}$ então existem $\left(\varepsilon_{i}\right)_{i \geq 2}$ e $\left(\varepsilon_{i}^{\prime}\right)_{i \geq l} \in \mathcal{N}, l<2$ tais que $z=\sum_{i=2}^{+\infty} \varepsilon_{i} \alpha^{i}=\sum_{i=l}^{+\infty} \varepsilon_{i}^{\prime} \alpha^{i}$ $e \varepsilon_{l}^{\prime} \neq 0$.

\section{Demonstração:}

1) Seja $z \in \partial \mathcal{R}=\overline{\mathcal{R}} \backslash \operatorname{int}(\mathcal{R})=\mathcal{R} \backslash \operatorname{int}(\mathcal{R})$. Então existe uma sequência $\left(z_{n}\right)_{n \geq 0}$ tal que

$$
\lim _{n \longrightarrow+\infty} z_{n}=z \quad e \quad z_{n} \notin \mathcal{R}, \forall n \geq 0
$$

Pela Proposição 2.1.5, existe uma sequência $\left(p_{n}\right)_{n \geq 0}$ de elementos de $\mathbb{Z}+\alpha \mathbb{Z}$ tal que para todo $n \geq 0, z_{n} \in \mathcal{R}+p_{n}, \forall n \geq 0$. Logo $\left(p_{n}\right)_{n \geq 0}$ é limitada. Como $\mathbb{Z}+\mathbb{Z} \alpha$ é um grupo discreto então $\left(p_{n}\right)_{n \geq 0}$ é uma sequência que possui um número finito de termos. Assim, existe uma subsequência $\left(p_{k_{n}}\right)_{n \geq 0}$ de $\left(p_{n}\right)_{n \geq 0}$ tal que para todo $n, p_{k_{n}}=p \in \mathbb{Z}+\mathbb{Z} \alpha$.

Como $z_{k_{n}} \in \mathcal{R}+p$ temos que $z=\lim z_{k_{n}} \in \mathcal{R}+p$ (pois $\mathcal{R}+p$ é fechado). Portanto,

$$
\partial \mathcal{R} \subset \bigcup_{p \in \mathbb{Z}+\mathbb{Z} \alpha} \mathcal{R} \cap(\mathcal{R}+p)
$$


Por outro lado, se $z \in \mathcal{R} \cap(\mathcal{R}+p), p \in \mathbb{Z}+\mathbb{Z} \alpha \backslash\{0\}$. Logo pela Proposição (2.1.6), $z \notin$ $\operatorname{int}(\mathcal{R})$, portanto, $z \in \partial \mathcal{R}$. Assim, $\partial \mathcal{R}=\bigcup_{p \in \mathbb{Z}+\mathbb{Z} \alpha} \mathcal{R} \cap(\mathcal{R}+p)=\bigcup_{p \in A} \mathcal{R} \cap(\mathcal{R}+p)$ onde $A=\{p \in \mathbb{Z}+\mathbb{Z} \alpha, \mathcal{R} \cap(\mathcal{R}+p) \neq \emptyset\}$.

Vamos provar que A é finito. De fato, seja $p \in A$ então existem $x, y \in \mathcal{R}$ tais que $p=x-y$. Logo $|p| \leq 2 M$ onde $M=\max \{|z|, z \in \mathcal{R}\}$. Assim $A \subset B(0,2 M)$. Portanto, A é finito pois $A \subset \mathbb{Z}+\mathbb{Z} \alpha$. Finalmente, a cardinalidade de $A$ é par pois se $u \in A$ então $-u \in A$.

Agora vamos provar que $\{1, \alpha, \alpha-1,-1,-\alpha,-(\alpha-1)\} \subset A$.

De fato, é fácil verificar que $-\alpha^{3}$ pode ser escrito das seguintes maneiras:

$$
\begin{array}{rcc}
-\alpha^{3} & = & (a-1) \sum_{i=1}^{\infty}\left(\alpha^{4 i+1}+\alpha^{4 i+2}\right) \\
& = & \alpha+(a-2) \alpha^{3}+(a-1) \sum_{i=1}^{\infty}\left(\alpha^{4 i}+\alpha^{4 i+1}\right) \\
& = & 1+(a-1) \alpha^{2}+(a-2) \alpha^{3}+(a-1) \sum_{i=1}^{\infty}\left(\alpha^{4 i}+\alpha^{4 i+1}\right)
\end{array}
$$

Logo, $-\alpha^{3} \in \mathcal{R} \cap(\mathcal{R}+\alpha) \cap(\mathcal{R}+1)$. Portanto, 1 e $\alpha$ pertencem a $A$.

Podemos também mostrar que

$$
\begin{array}{ccc}
w & = & \alpha-1+(a-1) \sum_{i=1}^{\infty}\left(\alpha^{4 i}+\alpha^{4 i+1}\right) \\
& = & (a-1) \sum_{i=1}^{\infty}\left(\alpha^{4 i-2}+\alpha^{4 i+1}\right) \\
& = & \alpha+(a-2) \alpha^{2}+\sum_{i=1}^{\infty}\left(\alpha^{4 i-1}+\alpha^{4 i+2}\right)
\end{array}
$$

Logo, $w \in \mathcal{R} \cap(\mathcal{R}+\alpha-1) \cap(\mathcal{R}+\alpha)$. Portanto, $\alpha-1$ pertence a A. Consequentemente concluimos a inclusão requerida.

2) Seja $z \in \partial \mathcal{R}$ então

$$
z=n+p \alpha+\sum_{i=2}^{+\infty} \varepsilon_{i} \alpha^{i}=\sum_{i=2}^{\infty} \varepsilon_{i}^{\prime} \alpha^{i},
$$

onde $\left(\varepsilon_{i}\right)_{i \geq 2},\left(\varepsilon_{i}^{\prime}\right)_{i \geq 2} \in \mathcal{N}$ e $n, p \in \mathbb{Z}$.

Caso 1.1 Supomos $n+p \beta<0$ e que o conjunto $\left\{i \geq 2, \varepsilon_{i} \neq 0\right\}$ é infinito.

Seja $t \geq 2$ tal que $n+p \beta+\sum_{i=2}^{t} \varepsilon_{i} \beta^{i}>0$. Logo

$$
n+p \beta+\sum_{i=2}^{t} \varepsilon_{i} \beta^{i}=\sum_{i=l}^{k} a_{i} \beta^{i}, \quad\left(a_{i}\right)_{l \leq i \leq k} \in \mathcal{N}_{f}, a_{k} \neq 0 .
$$

Temos $k \leq t$ pois senão $\sum_{i=2}^{t} \varepsilon_{i} \beta^{i}=-n-p \beta+\sum_{i=l}^{k} a_{i} \beta^{i}>\beta^{t+1}$, absurdo, logo

$$
n+p \beta+\sum_{i=2}^{t} \varepsilon_{i} \beta^{i}=\sum_{i=l}^{t} a_{i} \beta^{i} .
$$


Portanto, de (2.5),(2.6) e (2.4) temos que

$$
z=\sum_{i=l}^{t} a_{i} \alpha^{i}+\sum_{i=t+1}^{+\infty} \varepsilon_{i} \alpha^{i}=\sum_{i=2}^{+\infty} \varepsilon_{i}^{\prime} \alpha^{i}
$$

Afirmação: $a_{l} \ldots a_{t} \varepsilon_{t+1} \ldots \in \mathcal{N}$.

De fato, temos que $a_{l} \ldots a_{t} \in \mathcal{N}_{f}$ e $\varepsilon_{t+1} \varepsilon_{t+2} \ldots \in \mathcal{N}$.

Vamos supor $a_{l} \ldots a_{t} \varepsilon_{t+1} \ldots \notin \mathcal{N}$. Logo existe $x \in\left\{a_{t-2} a_{t-1} a_{t} \varepsilon_{t+1}, a_{t+1} a_{t} \varepsilon_{t+1} \varepsilon_{t+2} \ldots, a_{t} \varepsilon_{t+1} \varepsilon_{t+2} \varepsilon_{t+3}\right.$ tal que $x \geq 10(a-1)(a-1)\}$.

Supomos $x=a_{t-2} a_{t-1} a_{t} \varepsilon_{t+1}$. Logo $\varepsilon_{t+1}=a-1$, $a_{t}=a-1$. Agora vamos supor $a_{t-1}>0$. Então por (2.6) temos $\varepsilon_{t}=a-1$ e $\varepsilon_{t-1}>0$ pois senão a sequência $a_{l} \ldots a_{t}>$ lex $\varepsilon_{l} \ldots \varepsilon_{t} e$ assim, $\sum_{i=l}^{t} \varepsilon_{i} \beta^{i}<\sum_{i=l}^{t} a_{i} \beta^{i}$, absurdo.

Por outro lado, $\varepsilon_{t+1}=a-1 \operatorname{logo} \varepsilon_{t-2} \varepsilon_{t-1} \varepsilon_{t} \varepsilon_{t+1}>10(a-1)(a-1)$, absurdo.

Se $a_{t-1}=0$ e $a_{t-1}>1$ faz-se de modo análogo.

Ese $x \in\left\{a_{t-1} \ldots \varepsilon_{t+2}, a_{t} \ldots \varepsilon_{t+3}\right\}$ procedemos da mesma maneira.

Caso 1.2 $S e\left\{i, \varepsilon_{i} \neq 0\right\}$ é finito, contradiz a equação (2.4) pois implica que $\sum_{i=2}^{+\infty} \varepsilon_{i}^{\prime} \alpha^{i} \in \operatorname{int}(\mathcal{R})+n+p \alpha$ absurdo.

Caso 2 Supomos $n+p \beta>0$.

Seja $z_{k}=n+p \alpha+\sum_{i=2}^{k} \varepsilon_{i} \alpha^{i}$. Temos que $\lim _{k \longrightarrow+\infty} z_{k}=n+p \alpha+\sum_{i=2}^{+\infty} \varepsilon_{i} \alpha^{i}=\sum_{i=2}^{+\infty} \varepsilon_{i}^{\prime} \alpha^{i}=z$.

Por outro lado, para todo $k, z_{k}=\sum_{i=l_{k}}^{N_{k}} \varepsilon_{i, k}^{\prime \prime} \alpha^{i}, \varepsilon_{i, k}^{\prime \prime} \in \mathcal{D}^{\infty} e \varepsilon_{l_{k}, k}^{\prime \prime}>0$. Além do mais, $l_{k}<2$, pois senão $n=p=0$. Por outro lado, existe $s \in \mathbb{Z}, s<l_{k}, \forall k \in \mathbb{N}$ (pois z $z_{k}$ é limitado).

Logo, para todo $k, z_{k} \in \bigcup_{t=s}^{1} \mathcal{E}_{t}$ onde $\mathcal{E}_{t}=\left\{\sum_{i=t}^{+\infty} \varepsilon_{i} \alpha^{i},\left(\varepsilon_{i}\right)_{i \geq t} \in \mathcal{N}, \varepsilon_{t}>0\right\}$.

Como cada $\mathcal{E}_{t}$ é compacto (prova análoga a que foi feita para mostrar que $\mathcal{R}$ é compacto). Assim, $\bigcup_{t=s}^{1} \mathcal{E}_{t}$ compacto, portanto fechado. Então $z=\lim _{k \longrightarrow+\infty} z_{k} \in \bigcup_{t=s}^{1} \mathcal{E}_{t}$. Portanto, $z=\sum_{i=l}^{+\infty} \varepsilon_{i}^{\prime \prime} \alpha^{i},\left(\varepsilon_{i}^{\prime \prime}\right)_{l \leq i \leq+\infty} \in \mathcal{N}, l<2$.

\subsection{Caso $a=2$}

Teorema 2.2.1 $\mathcal{R}_{2}$ possui exatamente 6 vizinhos que são $\mathcal{R}_{2}+u$ onde $\pm u \in\{1, \alpha, \alpha-1\}$, isto é, $\forall u \in \mathbb{Z}+\mathbb{Z} \alpha \backslash\{0\}, \mathcal{R}_{2} \cap\left(\mathcal{R}_{2}+u\right) \neq \emptyset \Longleftrightarrow u \in\{ \pm 1, \pm \alpha, \pm(\alpha-1)\}$ 
Demonstração: Suponhamos, por absurdo, que $\mathcal{R} \cap(\mathcal{R}+p+q \alpha) \neq \emptyset$, então existe $z \in \mathcal{R}$ tal que

$$
z=\sum_{i=2}^{+\infty} \varepsilon_{i} \alpha^{i}=p+q \alpha+\sum_{i=2}^{+\infty} \varepsilon_{i}^{\prime} \alpha^{i}, \text { onde }\left(\varepsilon_{i}\right)_{i \geq 2},\left(\varepsilon_{i}^{\prime}\right)_{i \geq 2} \in \mathcal{N} .
$$

Por outro lado, agrupando os termos de quatro em quatro temos:

$$
\sum_{i=2}^{+\infty}\left(\varepsilon_{i}-\varepsilon_{i}^{\prime}\right) \alpha^{i}=\sum_{i=0}^{+\infty} y_{4 i} \alpha^{4 i+2}
$$

onde $y_{4 i}=\left(\varepsilon_{2+4 i}-\varepsilon_{2+4 i}^{\prime}\right)+\ldots+\left(\varepsilon_{5+4 i}-\varepsilon_{5+4 i}^{\prime}\right) \alpha^{3}$.

Assim,

$$
|p+q \alpha|^{2} \leq\left(\frac{k|\alpha|^{2}}{1-|\alpha|^{4}}\right)^{2}
$$

onde $k=\max \left\{\sum_{i=0}^{3}\left|\varepsilon_{i}-\varepsilon_{i}^{\prime}\right||\alpha|^{i}, \quad \varepsilon_{i}, \varepsilon_{i}^{\prime} \in\{0,1\}\right\}$. Podemos mostrar que $\left(\frac{k|\alpha|^{2}}{1-|\alpha|^{4}}\right)^{2}<3,1073$.

Por outro lado, considere $\alpha=c+d i$, onde $c=0,1225 \ldots$ e $d=0,7448 \ldots$ Logo $|p+q \alpha|^{2}=(p+$ $q c)^{2}+q^{2} d^{2}$. Portanto, $|q| \leq 2$ pois, caso contrário, se $|q|>3$ teríamos que $q^{2} d^{2}=4.999>3,1073$. Agora, vamos analisar os casos onde $q \in\{ \pm 2, \pm 1,0\}$ :

Caso 1: Se $q=-2$ então $p \in\{0,1\}$. De fato, $|p+q \alpha|^{2}=(p-2 c)^{2}+4 d^{2} \leq 3,1073 \Longrightarrow$ $(p-2 c)^{2} \leq 3,1073-2,2188=0,8885$. Os possiveis valores de $p$ são $\{0,1\}$ e as regióes associadas são $\mathcal{R}-2 \alpha$ e $\mathcal{R}+1-2 \alpha$.

Caso 2: Se $q=-1$ então $p \in\{-1,0,1\}$. De fato, neste caso temos: $|p+q \alpha|^{2}=(p-c)^{2}+d^{2} \leq$ $3,1073 \Longrightarrow(p-c)^{2} \leq 3,1073-0,5557=2,5525$. Logo, os possiveis valores de $p$ são $\{-1,0,1\}$ e as regiões associadas são: $\mathcal{R}-\alpha, \mathcal{R}-1-\alpha, \mathcal{R}+1-\alpha$.

Caso 3: Se $q=0$ então $p \in\{-1,0,1\}$. De fato, neste caso temos: $|p+q \alpha|^{2}=|p|^{2} \leq 3,1073 \Longrightarrow$ $p=-1,0,1$. Assim temos as regiões $\mathcal{R}, \mathcal{R}+1, \mathcal{R}-1$.

Caso 4: Se $q=1$ então $p \in\{-1,0,1\}$. De fato, neste caso temos: $|p+q \alpha|^{2}=(p+c)^{2}+d^{2} \leq$ $3,1073 \Longrightarrow(p+c)^{2} \leq 3,1073-0,5557=2,5525$. Logo, os possiveis valores de $p$ são $\{-1,0,1\} e$ as regiões associadas são: $\mathcal{R}+\alpha, \mathcal{R}+1+\alpha, \mathcal{R}-1+\alpha$.

Caso 5: Se $q=2$ então $p \in\{-1,0\}$. De fato, $|p+q \alpha|^{2}=(p+2 c)^{2}+4 d^{2} \leq 3,1073 \Longrightarrow$ $(p+2 c)^{2} \leq 3,1073-2,2188=0,8885$. Os possiveis valores de $p$ são $\{0,-1\}$ e as regiões associadas são $\mathcal{R}+2 \alpha$ e $\mathcal{R}-1+2 \alpha$.

Portanto, temos as seguintes regiões: $\mathcal{R} \pm 1, \mathcal{R} \pm \alpha, \mathcal{R} \pm(\alpha-1), \mathcal{R} \pm(1+\alpha), \mathcal{R} \pm 2 \alpha, \mathcal{R} \pm(-1+2 \alpha)$. Vamos eliminar estas 3 últimas regiões. De fato,

Suponhamos por absurdo que $\mathcal{R} \cap(\mathcal{R}+2 \alpha) \neq \emptyset$. Logo 
$2 \alpha=\sum_{i=2}^{+\infty}\left(\varepsilon_{i}-\varepsilon_{i}^{\prime}\right) \alpha^{i}$ onde $\left(\varepsilon_{i}\right),\left(\varepsilon_{i}^{\prime}\right) \in \mathcal{N}$. Portanto

$$
\left|2 \alpha+\left(\varepsilon_{2}^{\prime}-\varepsilon_{2}\right) \alpha^{2}\right|=\left|\sum_{i=3}^{+\infty}\left(\varepsilon_{i}-\varepsilon_{i}^{\prime}\right) \alpha^{i}\right|<|\alpha| \cdot 3,1073 \leq 1,3306
$$

Lembre que $|\alpha| \sim 0,42821$.

Por outro lado

$$
\left|2 \alpha+\left(\varepsilon_{2}^{\prime}-\varepsilon_{2}\right) \alpha^{2}\right| \subset\left\{|2 \alpha|,\left|2 \alpha+\alpha^{2}\right|,\left|2 \alpha-\alpha^{2}\right|\right\} \approx\{1,50 ; 1,69 ; 1,52\} .
$$

Logo, por (2.7) e (2.8) obtemos uma contradição.

Supomos, por absurdo, que $\mathcal{R} \cap(\mathcal{R}-1+2 \alpha) \neq \emptyset$. Assim, da mesma maneira que no caso precedente temos: $-1+2 \alpha=\sum_{i=2}^{+\infty}\left(\varepsilon_{i}-\varepsilon_{i}^{\prime}\right) \alpha^{i} \operatorname{logo}$

$$
\left|-1+2 \alpha+\left(\varepsilon_{2}^{\prime}-\varepsilon_{2}\right) \alpha^{2}\right|=\left|\sum_{i=3}^{+\infty}\left(\varepsilon_{i}-\varepsilon_{i}^{\prime}\right) \alpha^{i}\right|<|\alpha| \cdot 3,1073 \leq 1,3306
$$

Por outro lado,

$\left|-1+2 \alpha+\left(\varepsilon_{2}^{\prime}-\varepsilon_{2}\right) \alpha^{2}\right| \subset\left\{|-1+2 \alpha|,\left|-1+2 \alpha+\alpha^{2}\right|,\left|-1+2 \alpha-\alpha^{2}\right|\right\} \approx\{1,67 ; 2,11 ; 1,32\}$.

Logo, por (2.9) e (2.10) obtemos uma contradição.

Finalmente, supomos novamente por absurdo que $\mathcal{R} \cap \mathcal{R}+1+\alpha \neq \emptyset$. Então, $1+\alpha=\sum_{i=2}^{+\infty}\left(\varepsilon_{i}-\right.$ $\left.\varepsilon_{i}^{\prime}\right) \alpha^{i}$ e assim

$$
\left|1+\alpha+\left(\varepsilon_{2}^{\prime}-\varepsilon_{2}\right) \alpha^{2}\right|=\left|\sum_{i=3}^{+\infty}\left(\varepsilon_{i}-\varepsilon_{i}^{\prime}\right) \alpha^{i}\right|<|\alpha| \cdot 3,1073 \leq 1,3306
$$

Por outro lado,

$\left|-1+\alpha+\left(\varepsilon_{2}^{\prime}-\varepsilon_{2}\right) \alpha^{2}\right| \subset\left\{|1+\alpha|,\left|1+\alpha+\alpha^{2}\right|,\left|1+\alpha-\alpha^{2}\right|\right\} \approx\{1,34 ; 1,09 ; 1,75\}$.

Logo, por (2.11) e (2.12) obtemos uma contradição. 


\section{Capítulo}

3

\section{O autômato que reconhece a fronteira de}

\section{$\mathcal{R}$}

No capítulo anterior, a mostramos que todo elemento da fronteira de $\mathcal{R}_{a}$ tem pelo menos duas $\alpha$-representaçôes. Definimos um autômato que fornece todos os números complexos que tem pelo menos duas $\alpha$-representações. Com isso provamos que para todo $a \geq 2$

$$
\partial \mathcal{R}_{a}=\bigcup_{u \in B} \mathcal{R}_{a} \cap\left(\mathcal{R}_{a}+u\right)
$$

onde $B=\{ \pm 1, \pm \alpha, \pm(\alpha-1)\}$.

Definição 3.0.2 Um autômato finito é uma terna $(S, A, C)$ onde $A$ é o alfabeto, $S$ o conjunto de estados e $C$ um subconjunto de $S \times A \times S$.

Seja $\left(a_{n}\right)_{n \geq 0}$ uma sequência de elementos de A. Dizemos que $\left(a_{n}\right)_{n \geq 0}$ é um caminho infinito no autômato se existir uma sequência $\left(s_{n}\right)_{n \geq 0}$ onde $s_{n} \in S, \forall n \geq 0$ tal que $\left(s_{n-1}, a_{n}, s_{n}\right) \in C, \forall n \geq 1$.

Uma palavra $a_{1} \ldots a_{n}$ (respectivamente, uma palavra infinita $a_{1} \ldots a_{n} \ldots$ ) é reconhecivel pelo autômato $(S, A, C)$, se existir uma palavra $s_{0} \ldots s_{n} \in S, s_{0} \in I$ (resp. uma sequência $s_{n} \in A^{\mathbb{N}}$ ) tal que $\left(s_{i-1}, a_{i}, s_{i}\right) \in C$, para $i=1, \ldots, n$ (resp. para todo $i \in \mathbb{N}$ ).

Dizemos que uma sequência $\left(a_{n}\right)_{n \geq 0}$ é reconhecida pelo autômato $(S, A, C)$ se existir uma sequência $\left(s_{n}\right)_{n \geq 0} \in A^{\mathbb{N}}$ tal que $\left(s_{i-1}, a_{i}, s_{i}\right) \in C, \forall i \geq 1$.

\subsection{Relação com as representações impróprias na base $\alpha$}

Como o módulo de a é inferior à 1 e 0 pertence à int $(\mathcal{R})$, para todo número complexo $z$ existe um inteiro $k$ tal que $\alpha^{k} z \in \mathcal{R}$. Logo, todo número complexo $z$ se escreve na base $\alpha$ como $z=$ 
$\sum_{i=l}^{\infty} \varepsilon_{i} \alpha^{i}, l \in \mathbb{Z}$ e $\left(\varepsilon_{i}\right)_{i \geq l} \in \mathcal{N}$. A sequência $\left(\varepsilon_{i}\right)_{i \geq l}$ é chamada $\alpha$-representação de z. Em virtude do item (ii) do Lema (2.1.4) todo ponto da fronteira tem pelo menos duas $\alpha$-representações. Esses números complexos são caracterizados pelo teorema que foi provado por Thurston [28].

Teorema 3.1.1 [28] Existe um autômato finito $\mathcal{B}$ tal que para todo $\left(a_{i}\right)_{i \geq l}$ e $\left(b_{i}\right)_{i \geq l}$ dois elementos distintos de $\mathcal{N}$ temos que $\sum_{i=l}^{\infty} a_{i} \alpha^{i}=\sum_{i=l}^{\infty} b_{i} \alpha^{i}$ se, e somente se, a sequência $\left(\left(a_{i}, b_{i}\right)\right)_{i \geq l}$ é reconhecido pelo autômato $\mathcal{B}$.

Porém a prova de Thurston não fornece explicitamente os estados.

\subsubsection{Definição do autômato que reconhece os pontos com no mínimo duas ex- pansões}

Na sequência mostraremos como construir o autômato $\mathcal{A}$ que caracteriza a fronteira de $\mathcal{R}$. Provaremos na sessão seguinte que os estados do autômato $\mathcal{A}$ são (ver Teorema 3.2.1) $S=S_{a}=$ $\left\{0, \pm \alpha, \pm \alpha^{2}, \pm\left(\alpha-\alpha^{2}\right), \pm\left(1+(a-1) \alpha^{2}\right), \pm\left(1+(a-2) \alpha^{2}\right), \pm\left(1-\alpha+(a-1) \alpha^{2}\right), \pm\left(1-2 \alpha+a \alpha^{2}\right)\right\}$.

Sejam s e $t$ dois estados. O conjunto das arestas é $(s,(c, d), t) \in S \times\{0,1, \ldots, a-1\}^{2} \times S$ de modo que satisfaça $t=\frac{s}{\alpha}+(c-d) \alpha^{2}$. O conjunto de estados iniciais é $\{(0,(0,0), 0)\}$.

Vamos explicar como usar este autômato. Seja $\varepsilon=\left(\varepsilon_{i}\right)_{i \geq l}$ e $\varepsilon^{\prime}=\left(\varepsilon_{i}^{\prime}\right)_{i \geq l}$ em $\mathcal{D}^{\infty}, x=\sum_{i=l}^{\infty} \varepsilon_{i} \alpha^{i}$ e $y=\sum_{i=l}^{\infty} \varepsilon_{i}^{\prime} \alpha^{i}$. Supomos que $x=y$. Para todo $k \geq l$ temos

$$
A_{k}\left(\varepsilon, \varepsilon^{\prime}\right)=\alpha^{-k+2} \sum_{i=l}^{k}\left(\varepsilon_{i}-\varepsilon_{i}^{\prime}\right) \alpha^{i}
$$

Na próxima subsessão vamos provar que para todo $A_{k}, k \in \mathbb{N}$, pertence a $S$. Claramente, para todo $k \geq l$,

$$
A_{k+1}\left(\varepsilon, \varepsilon^{\prime}\right)=\frac{A_{k}\left(\varepsilon, \varepsilon^{\prime}\right)}{\alpha}+\left(\varepsilon_{k+1}-\varepsilon_{k+1}^{\prime}\right) \alpha^{2}
$$

Seja s o menor inteiro tal que $\varepsilon_{s} \neq \varepsilon_{s}^{\prime}$. Assim $A_{i}\left(\varepsilon, \varepsilon^{\prime}\right)=0$ para $i \in\{l, \ldots, s-1\}$. Suponhamos que $\varepsilon_{s}>\varepsilon_{s}^{\prime}$. Então, $A_{s}=\left(\varepsilon_{s}^{\prime}-\varepsilon_{s}\right) \alpha^{2}=\alpha^{2}$. Por (3.2) deduzimos que $A_{s+1}\left(\varepsilon, \varepsilon^{\prime}\right)=\alpha+\left(\varepsilon_{s+1}-\right.$ $\left.\varepsilon_{s+1}^{\prime}\right) \alpha^{2}$ o qual deve pertencer a $S_{a}$. Assim $A_{s+1}\left(\varepsilon, \varepsilon^{\prime}\right)=\alpha-\alpha^{2}$ se $\left(\varepsilon_{s+1}, \varepsilon_{s+1}^{\prime}\right)=(t-1, t)$, onde $1 \leq t \leq a-1$ ou $A_{s+1}\left(\varepsilon_{i}, \varepsilon_{i}^{\prime}\right)=\alpha$ se $\left(\varepsilon_{s+1}, \varepsilon_{s+1}^{\prime}\right)=(t, t)$, onde $0 \leq t \leq a-1$. Logo $\left(\alpha^{2},(t-1, t), \alpha-\alpha^{2}\right)$, é uma aresta que liga o estado $\alpha^{2}$ ao estado $\alpha-\alpha^{2}$ e $\left(\alpha^{2},(t, t), \alpha\right)$, é uma aresta que liga o estado $\alpha^{2}$ ao estado $\alpha$. Como o conjunto de estados $S$ é finito, obtemos um autômato finito (ver Figura). 


\subsection{Caracterização dos pontos com duas expansões}

Para todo $\varepsilon=\left(\varepsilon_{i}\right)_{i \geq l}$ e $\varepsilon^{\prime}=\left(\varepsilon_{i}^{\prime}\right)_{i \geq l}$ em $\mathcal{D}^{\infty}$, seja

$$
S\left(\varepsilon, \varepsilon^{\prime}\right)=\left\{A_{k}\left(\varepsilon, \varepsilon^{\prime}\right) ; k \geq l\right\}=\left\{\alpha^{-k+2} \sum_{i=l}^{k}\left(\varepsilon_{i}-\varepsilon_{i}^{\prime}\right) \alpha^{i} ; k \geq l\right\} .
$$

$e$

$$
\mathcal{D}^{\infty}:=\left\{\left(a_{i}\right)_{i \geq k}, k \in \mathbb{Z}, \forall n \geq k,\left(a_{i}\right)_{k \leq i \leq n} \text { é uma } R \text {-representação }\right\}
$$

isto é, $a_{n} a_{n-1} a_{n-2} a_{n-3}<_{l e x}(a-1)(a-1) 01, \forall n \geq k$ onde $a_{k-1}=a_{k-2}=a_{k-3}=0$.

Teorema 3.2.1 Sejam $x=\sum_{i=l}^{\infty} \varepsilon_{i} \alpha^{i}, y=\sum_{i=l}^{\infty} \varepsilon_{i}^{\prime} \alpha^{i}$, onde $\varepsilon=\left(\varepsilon_{i}\right)_{i \geq l}$ e $\varepsilon^{\prime}=\left(\varepsilon_{i}^{\prime}\right)_{i \geq l}$ em $\mathcal{D}^{\infty}$. Então, $x=y$ se, e somente se, $S\left(\varepsilon, \varepsilon^{\prime}\right)$ é finito. Além disso, $S\left(\varepsilon, \varepsilon^{\prime}\right) \subset S_{a}=\left\{0, \pm \alpha, \pm \alpha^{2}, \pm(\alpha-\right.$ $\left.\left.\alpha^{2}\right), \pm\left(1+(a-1) \alpha^{2}\right), \pm\left(1+(a-2) \alpha^{2}\right), \pm\left(1-\alpha+(a-1) \alpha^{2}\right), \pm\left(1-2 \alpha+a \alpha^{2}\right)\right\} . E$

$$
S_{a}=\bigcup_{\left(\varepsilon, \varepsilon^{\prime}\right) \in \Delta} S\left(\varepsilon, \varepsilon^{\prime}\right)
$$

onde $\Delta=\left\{\left(\left(\varepsilon_{i}\right)_{i \geq l},\left(\varepsilon_{i}^{\prime}\right)_{i \geq l}\right) \in \mathcal{D}^{\infty} \times \mathcal{D}^{\infty} ; \sum_{i=l}^{\infty} \varepsilon_{i} \alpha^{i}=\sum_{i=l}^{\infty} \varepsilon_{i}^{\prime} \alpha^{i}\right\}$.

Demonstração do Teorema 3.2.1: Supomos que $S\left(\varepsilon, \varepsilon^{\prime}\right)$ é finito, logo existe um número real $M>0$ tal que $\left|\sum_{i=l}^{k} \varepsilon_{i} \alpha^{i}-\sum_{i=l}^{k} \varepsilon_{i}^{\prime} \alpha^{i}\right| \leq M|\alpha|^{k-2}$, para todo $k \geq l$. Como $0<|\alpha|<1$, então, fazendo $k$ tender $a+\infty$ temos $\sum_{i=l}^{+\infty} \varepsilon_{i} \alpha^{i}=\sum_{i=l}^{+\infty} \varepsilon_{i}^{\prime} \alpha^{i}$.

Vamos provar a recíproca. Sejam $x=\sum_{i=l}^{\infty} \varepsilon_{i} \alpha^{i}$ e $y=\sum_{i=l}^{\infty} \varepsilon_{i}^{\prime} \alpha^{i} \operatorname{com} \varepsilon=\left(\varepsilon_{i}\right)_{i \geq l}$ e $\varepsilon^{\prime}=\left(\varepsilon_{i}^{\prime}\right)_{i \geq l}$ em $\mathcal{D}^{\infty}$. Suponhamos $x=y$. Vamos provar que $A_{k}=A_{k}\left(\varepsilon, \varepsilon^{\prime}\right)$ pertence a $S$ para todo $k \geq l$. Como $x=y$, então para todo $k \geq l$, temos

$$
A_{k}=\sum_{i=k+1}^{\infty}\left(\varepsilon_{i}^{\prime}-\varepsilon_{i}\right) \alpha^{i-k+2}=\sum_{i=3}^{\infty}\left(\varepsilon_{i+k-2}^{\prime}-\varepsilon_{i+k-2}\right) \alpha^{i} \in \mathcal{R}-\mathcal{R}
$$

Vamos fixar $k \geq l$ e assumir que $A_{k} \neq 0$. A partir de (3.1) e do fato que $\alpha$ é um inteiro algébrico de grau 3, deduzimos

$$
A_{k}=n_{k} \alpha^{2}+p_{k} \alpha+q_{k}, \text { onde } n_{k}, p_{k}, q_{k} \in \mathbb{Z}
$$


Como $n_{k} \beta^{2}+p_{k} \beta+q_{k}$ ou $-\left(n_{k} \beta^{2}+p_{k} \beta+q_{k}\right)$ pertencem a $\mathbb{Z}[\beta] \cap \mathbb{R}^{+}$, deduzimos do item (iv) do Lema 2.1.4, que existe $\left(c_{i}\right)_{s_{k} \leq i \leq m_{k}} \in \mathcal{N}_{f}$ tal que $c_{m_{k}}>0$ e

$$
n_{k} \beta^{2}+p_{k} \beta+q_{k}= \pm \sum_{i=s_{k}}^{m_{k}} c_{i} \beta^{i}
$$

Supomos que é igual a $\sum_{i=s_{k}}^{m_{k}} c_{i} \beta^{i}$. O outro caso pode ser tratado do mesmo modo. Como $\beta$ e $\alpha$ são conjugados algebricamente, a partir de (3.1), (3.4) e (3.5) obtemos

$$
\beta^{-k+2} \sum_{i=l}^{k} \varepsilon_{i} \beta^{i}=\beta^{-k+2} \sum_{i=l}^{k} \varepsilon_{i}^{\prime} \beta^{i}+\sum_{i=s_{k}}^{m_{k}} c_{i} \beta^{i} .
$$

A partir do item (vi) do Lema 2.1.4, $\beta^{-k+2} \sum_{i=l}^{k} \varepsilon_{i} \beta^{i}<\beta^{3}$, consequentemente $m_{k} \leq 2$. Colocando $c_{i}=0$ para todo $i>m_{k}$, temos $n_{k} \beta^{2}+p_{k} \beta+q_{k}=\sum_{i=s_{k}}^{2} c_{i} \beta^{i}$. Como $\beta$ é um número de Pisot, temos por proposição em [14] que existe um inteiro $s=s(a)$ tal que $s \leq s_{k}$. Portanto,

$$
A_{k}=\sum_{i=s}^{2} c_{i} \alpha^{i}
$$

Logo

$$
S_{a} \subset\left\{\sum_{i=s}^{2} c_{i} \alpha^{i},\left(c_{i}\right)_{s \leq i \leq 2} \in \mathcal{N}_{f}\right\}
$$

Observe que se

$$
A_{k}=\sum_{i=s}^{2} c_{i} \alpha^{i} \text { então } \widetilde{A}_{k}=\sum_{i=s}^{2} c_{i} \beta^{i}<\beta^{3}
$$

Para provar que $S_{a}=\left\{0, \pm \alpha, \pm \alpha^{2}, \pm\left(\alpha-\alpha^{2}\right), \pm\left(1+(a-1) \alpha^{2}\right), \pm\left(1+(a-2) \alpha^{2}\right), \pm(1-\alpha+\right.$ $\left.\left.(a-1) \alpha^{2}\right), \pm\left(1-2 \alpha+a \alpha^{2}\right)\right\}$, precisamos dos seguinte Lema:

Lema 3.2.2 Sejam $n, p, q \in \mathbb{Z}$ e $z=n+p \alpha+q \alpha^{2}$ um elemento de $S_{a}$ então $|n| \leq 1$.

Demonstração: Sejam $S=\left\{A_{k}=n_{k}+p_{k} \alpha+q_{k} \alpha^{2}, k \geq 1\right\}$ e $n=\max \left\{\left|n_{k}\right|, k \geq 0\right\}$. Supomos $n \geq 2$.

Seja $k \in \mathbb{N}$ tal que $A_{k}=n+p \alpha+q \alpha^{2}, p, q \in \mathbb{Z}$. Logo $A_{k+1}=\frac{n}{\alpha}+(p+q) \alpha+d \alpha^{2},|d| \leq a-1$. Portanto,

$$
A_{k+1}=(p+n)+(q-n a) \alpha+(d+n) \alpha^{2} .
$$


Assim, $-n \leq p+n \leq n, \log o-2 n \leq p \leq 0$.

Por outro lado,

$$
A_{k+2}=(q-n a+p+n)+(d+n-a(p+n)) \alpha+(f+p+n) \alpha^{2},|f| \leq a-1 .
$$

Logo, $-n \leq q-n a+p+n \leq n$. Portanto, $n(a-2)-p \leq q$.

Por outro lado,

$$
\begin{array}{rlc}
\widetilde{A}_{k} & = & n+p \beta+q \beta^{2} \\
& = & 1-\beta+a \beta^{2}+(n-1)+(p+1) \beta+(q-a) \beta^{2} \\
& \geq & \beta^{3}+(n-1)+(p+1) \beta+(n(a-2)-p-a) \beta^{2} .
\end{array}
$$

Como $n \geq 2$ então $n(a-2)-p-a \geq a-4-p$.

$\log O$

$$
\widetilde{A}_{k} \geq \beta^{3}+(n-1)+(p+1) \beta+(a-4-p) \beta^{2}
$$

Caso 1 Se $a \geq 4$ então $a-4-p \geq 0$, logo

$$
\begin{aligned}
\widetilde{A}_{k} & \geq \quad \beta^{3}+(n-1)+(p+1+a-4-p) \beta \\
& =\beta^{3}+(n-1)+(a-3) \beta>\beta^{3}, \quad \text { o que não ocorre devido a equação } 3.9
\end{aligned}
$$

Caso 2 Se $a=3 e-2 n \leq p<0$, então $a-4-p \geq 0$ e obtemos $\widetilde{A}_{k} \geq \beta^{3}$.

Caso 2.1 Se $a=3$ e $p=0$ então

$A_{k+3}=(d+n-a(p+n)+q-n a+p+n)+((f+p+n)-a(q-n a+p+n)) \alpha+(g+q-n a+p+n) \alpha^{2},|g| \leq a-1$

$\operatorname{Logo}$,

$$
\begin{array}{cccc}
-n & \leq & d+n-a(p+n)+q-n-1+p+n \leq n \\
n(2 a-3)+p(a-1) & \leq & q-a \\
3 n-5 & \leq & q-3
\end{array}
$$

Das equações (3.10) e (3.12) temos

$$
\widetilde{A}_{k} \geq \beta^{3}+(n-1)+\beta+(3 n-5) \beta^{2}>\beta^{3}, \text { pois } n \geq 2 \text {. }
$$

Caso 3 Se $a=2$ e $p<-2$ então $a-4-p \geq 0$ e segue que 


$$
\begin{array}{rlc}
\widetilde{A}_{k} & \geq & \beta^{3}+(n-1)+(p+1) \beta+(-2-p) \beta^{2} \\
& = & \beta^{3}+(-2-p)+(n-1+2+p)+((p+1)+2(-2-p)) \beta \\
& = & \beta^{3}+(-2-p)+(n+1+p)+(-3-p) \beta
\end{array}
$$

Caso 3.1 Se $-2 n \leq p \leq-3$ então $\widetilde{A}_{k} \geq \beta^{3}+(-2-p)+(n-2) \geq \beta^{3}$, o que não ocorre devido a equação 3.9.

Caso 3.2 Se $a=2$ e $-2 \leq p \leq 0$ então $A_{k}=n+q \alpha^{2}$ ou $A_{k}=n-\alpha+q \alpha^{2}$ ou $A_{k}=n-2 \alpha+q \alpha^{2}$.

Caso 3.2.1 Se $A_{k}=n+q \alpha^{2}, n \geq 2$ então

$$
A_{k+1}=n+(q-n a) \alpha+(d+n) \alpha^{2}
$$

Portanto, (ver casos 3 e 3.1) temos $q-n a=0$ ou -1 ou -2 . Assim $q=n a$ ou $q=n a-1$ ou $q=n a-2$. Logo

$$
\begin{gathered}
\widetilde{A}_{k}=n+(n a-1) \beta^{2}>1-\beta+a \beta^{2}=\beta^{3} \text { ou } \\
\widetilde{A}_{k}=n+n a \beta^{2}>\beta^{3} \text { ou } \\
\widetilde{A}_{k}=n+(n a-2) \beta^{2} \geq n+2 \beta^{2}>1-\beta+a \beta^{2}=\beta^{3}
\end{gathered}
$$

os quais não ocorrem devido a equação 3.9.

Caso 3.2.2 Se $A_{k}=n-\alpha+q \alpha^{2}$ então

$$
\begin{gathered}
A_{k+1}=(n-1)+(q-n a) \alpha+(d+n) \alpha^{2} e \\
A_{k+2}=(q-n a+n-1)+(d+n-a(n-1)) \alpha+(f+n-1) \alpha^{2} . \\
\text { Temos }-n \leq q-n a+n-1 \leq n \Longrightarrow 1=n(a-2)+1 \leq q .
\end{gathered}
$$

Caso 3.2.2.1 Se $q \geq 2=a$ então

$$
\widetilde{A}_{k}=n-\beta+q \beta^{2} \geq n-\beta+a \beta^{2} \geq \beta^{3}
$$

Caso 3.2.2.1.1 Se $q=1$ então $A_{k+2}=-n+[d+n-a(n-1)] \alpha+(f+n-1) \alpha^{2}$. Como $A_{k+2} \in S_{a}$ então $-A_{k+2} \in S_{a}$. Temos

$$
-A_{k+2}=n+[a(n-1)-n-d] \alpha+(-f-n+1) \alpha^{2} .
$$

Do que foi feito anteriormente temos a $(n-1)-n-d \in\{1,-2\}$.

Se $a(n-1)-n-d=-1$ então $d=n-1 \leq 1$, logo $n \leq 2$.

Se $a(n-1)-n-d=-2$ então $d=n-1 \leq 1$, logo $n \leq 1$, absurdo pois $n \geq 2$. 
Logo $n=2$ ou $n=3$, portanto, $A_{k}=2-\alpha+\alpha^{2}$ ou $A_{k}=3-\alpha+\alpha^{2}$.

Caso 3.2.2.1.1.1 Se $A_{k}=2-\alpha+\alpha^{2}$ então

$$
\begin{array}{rlc}
A_{k+1} & = & 1+(1-2 a) \alpha+(d+2) \alpha^{2} \\
& = & 1-3 \alpha+(d+2) \alpha^{2}
\end{array}
$$

$$
\begin{array}{rlr}
A_{k+2} & = & -2+(d+2-a) \alpha+(f+1) \alpha^{2} \\
& = & -2+d \alpha+(f+1) \alpha^{2},
\end{array}
$$

Portanto, $A_{k+2}=-2+\alpha-\alpha^{2} \operatorname{logo} d=1$ e $f=-2$, o que não ocorre pois $|f| \leq a-1=1$ ou $A_{k+2}=-2+2 \alpha-o \alpha^{2}$ E assim $d=2$, o que não ocorre.

Caso 3.2.3 Se $A_{k}=n-2 \alpha+q \alpha^{2}$ então $A_{k+1}=(n-2)+(q-n a) \alpha+(d+n) \alpha^{2} e$

$$
A_{k+2}=(q-n a+n-2)+(d+n-a(n-2)) \alpha+(f+n-2) \alpha^{2},|f| \leq a-1
$$

Assim, $-n \leq q-n a+n-2 \leq n, \operatorname{logo} n(a-2) \leq q-2$ então $n(a-2)+2 \leq q$, portanto, $2 \leq q$

Caso 3.2.3.1 Se $q \geq 3$ então

$$
\begin{aligned}
& \widetilde{A}_{k}=\quad n-2 \beta+q \beta^{2} \geq n-2 \beta+(a+1) \beta^{2} \\
& =n-\beta+a \beta^{2}+\beta^{2}-\beta \\
& =\beta^{3}+\underbrace{\left(\beta^{2}-\beta\right)}_{>0}>\beta^{3} \text {, o que não ocorre devido a equação (3.9). }
\end{aligned}
$$

Caso 3.2.3.2 Se $q=2$ então $A_{k}=n-2 \alpha+2 \alpha^{2}$ e $A_{k+2}=-n+(d-n+4) \alpha+(f+n-2) \alpha^{2}$.

Logo, $d-n+4=2$ e assim $d=n-2 \leq 1$ e, portanto, $n \leq 3$.

Caso 3.2.3.2a) Se $n=2$ então $A_{k}=2-2 \alpha+2 \alpha^{2}, A_{k+1}=(2-2 a) \alpha+(d+2) \alpha^{2}=-2 \alpha+(d+2) \alpha^{2}$ e assim

$$
A_{k+2}=-2+(d+2) \alpha+f \alpha^{2} \text { e assim } d=0 \text { e } f=2 \text { o que não ocorre. }
$$

Caso 3.2.3.2b) Se $n=3$ então $A_{k}=3-2 \alpha+2 \alpha^{2}, A_{k+1}=1+(2-3 a) \alpha+(d+3) \alpha^{2}=1-4 \alpha+(d+3) \alpha^{2}$ e assim $A_{k+2}=-3+(d+3-a) \alpha+(f+1) \alpha^{2} \operatorname{logo} d+3-a=2$ então $d=a-1=1 e$ $f+1=-2$ portanto $f=-3$ o que não ocorre.

Agora vamos provar que $S_{a}=\left\{0, \pm \alpha, \pm \alpha^{2}, \pm\left(\alpha-\alpha^{2}\right), \pm\left(1+(a-1) \alpha^{2}\right), \pm\left(1+(a-2) \alpha^{2}\right), \pm(1-\alpha+(a-\right.$ 1) $\left.\left.\alpha^{2}\right), \pm\left(1-2 \alpha+a \alpha^{2}\right)\right\}$. 
Seja $A_{k}=n+p \alpha+q \alpha^{2} \in S_{a}$, logo pelo Lema anterior (3.2.2) temos $|n| \leq 1$.

Caso 1 Supomos que $n=1$, então

$A_{k}=1+p \alpha+q \alpha^{2}$ logo $A_{k+1}=(p+1)+(q-a) \alpha+(d+1) \alpha^{2}$, onde $|d| \leq a-1$.

Assim

$$
-1 \leq p+1 \leq 1 \text { e, então } p \in\{-2,-1,0\}
$$

Caso 1.1 Se $p=0$ então $A_{k+1}=1+(q-a) \alpha+(d+1) \alpha^{2}$. Logo, pela relação (3.13) $-2 \leq q-a \leq 0$ então $a-2 \leq q \leq a$.

Se $q=a$ então $\widetilde{A}_{k}=1+a \beta^{2}>\beta^{3}$, o que não ocorre. Portanto, $q=a-1$ ou $q=a-2$. Assim temos os estados $A_{k}=1+(a-1) \alpha^{2}$ e $A_{k}=1+(a-2) \alpha^{2}$.

Caso 1.2 Se $p=-1$ então $A_{k+1}=(q-a) \alpha+(d+1) \alpha^{2}$ e $A_{k+2}=(q-a)+(d+1) \alpha+e \alpha^{2},|e| \leq a-1$. Assim, da relação (3.13) temos $-1 \leq q-a \leq 1$ então $q=a-1, a, a+1$. Para os casos $q=a e$ $q=a+1$ temos $\widetilde{A}_{k} \geq \beta^{3}$. Logo $q=a-1$ e, portanto, obtemos o estado $A_{k}=1-\alpha+(a-1) \alpha^{2}$.

Caso 1.3 Se $p=-2$ então $A_{k+1}=-1+(q-a) \alpha+(d+1) \alpha^{2}$ e $A_{k+2}=(q-a-1)+(d+1+a) \alpha+$ $(e-1) \alpha^{2},|e| \leq a-1$. Assim, da relação (3.13) temos $-1 \leq q-a-1 \leq 1$ logo $a \leq q \leq a+2$. Assim, se $q=$ a obtemos o estado $A_{k}=1-2 \alpha+a \alpha^{2}$.

Se $q=a+1$ então $\widetilde{A}_{k}=1-2 \beta+(a+1) \beta^{2}=1-\beta+a \beta^{2}-\beta+\beta^{2}>\beta^{3}$, o que não ocorre. Se $q=a+2$ então $\widetilde{A}_{k}=1-2 \beta+(a+2) \beta^{2}=1-\beta+a \beta^{2}-\beta+2 \beta^{2}>\beta^{3}$, o que não ocorre.

Caso 2 Supomos $n=0$ então $A_{k}=p \alpha+q \alpha^{2}$ e $A_{k+1}=p+q \alpha+d \alpha^{2} \operatorname{logo} A_{k+2}=q+d \alpha+2 \alpha^{2}$ assim $q=0, \pm 1$. Portanto, $A_{k}=0, \pm \alpha^{2}$.

Caso 2.1 Se $p=1$ então $A_{k+1}=1+q \alpha+d \alpha^{2}$, logo pelo Caso 1 temos $q=0,-1,-2$ e, portanto, obtemos os estados $A_{k}=\alpha, \alpha-\alpha^{2}, \alpha-2 \alpha^{2}$. Suponhamos que $A_{k}=\alpha-2 \alpha^{2} \in S_{a}$ então $A_{k+1}=1-2 \alpha+d \alpha^{2} \in S_{a}$. Mas como foi visto anteriormente $A_{k}=1-2 \alpha+d \alpha^{2}=1-2 \alpha+a \alpha^{2}$ $e$, portanto, $d=a$, o que não ocorre, pois $d=\varepsilon-\varepsilon^{\prime}$ onde $\varepsilon, \varepsilon^{\prime} \in\{0,1, \ldots, a-1\}$. 


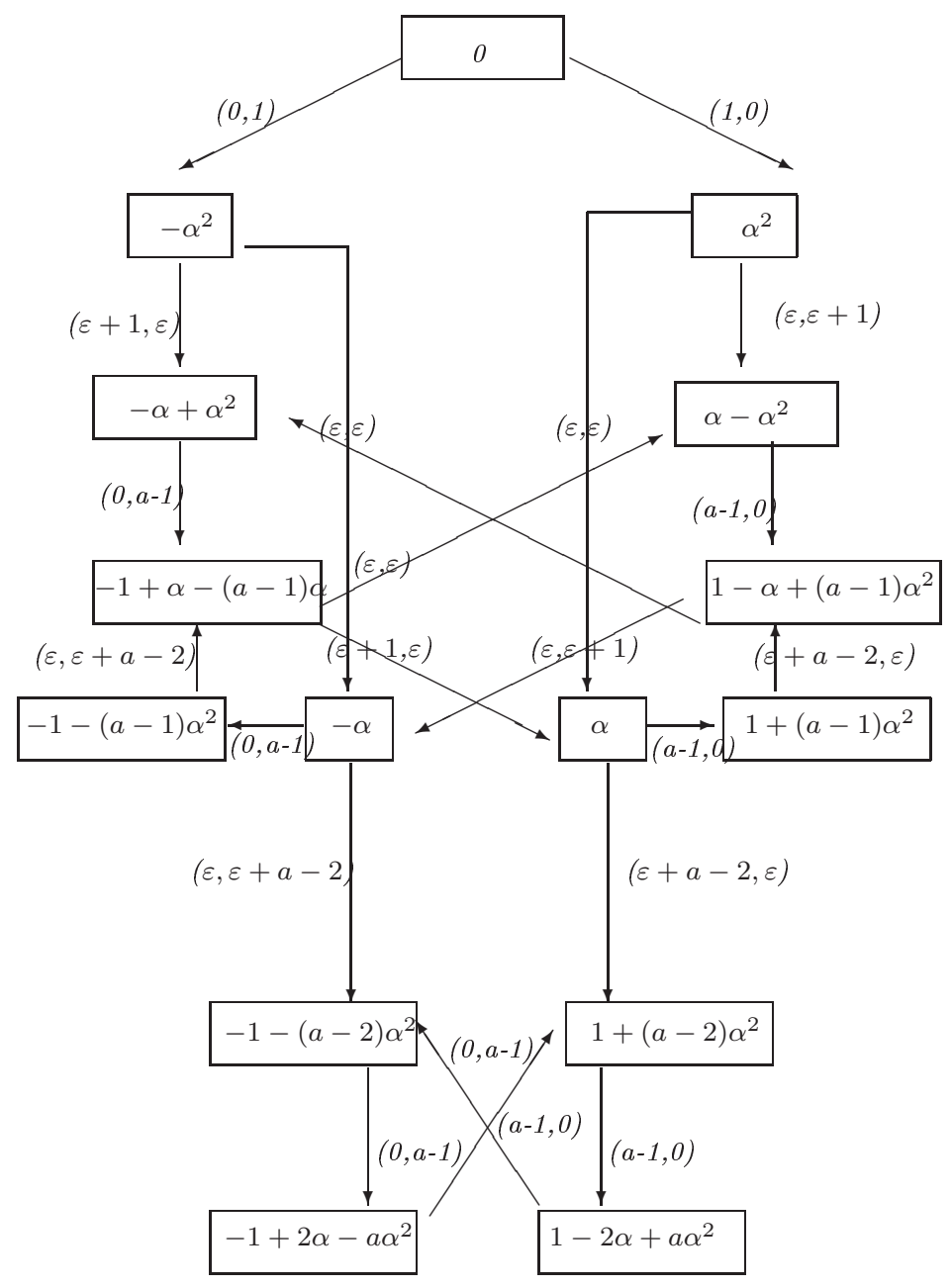

(Autômato: a qualquer)

Teorema 3.2.3 Para todo a $\geq 2$ temos

$$
\partial \mathcal{R}_{a}=\bigcup_{u \in B} \mathcal{R}_{a} \cap\left(\mathcal{R}_{a}+u\right)
$$

onde $B=\{ \pm 1, \pm \alpha, \pm(\alpha-1)\}$.

Demonstração do Teorema: $\quad$ Pelo item (1) do Teorema (2.1.8), temos que $\bigcup_{u \in B} \mathcal{R} \cap(\mathcal{R}+$ u) $\subset \partial \mathcal{R}$. 
Seja $z \in \partial \mathcal{R}$ então pelo item (2) do Teorema (2.1.8) temos

$$
z=\sum_{i=2}^{\infty} \varepsilon_{i} \alpha^{i}=\sum_{i=l}^{\infty} \varepsilon_{i}^{\prime} \alpha^{i} \text { onde } l<2 \text { e } \varepsilon_{l}^{\prime} \neq 0
$$

Vamos supor $\varepsilon_{2}>0$. Logo $r=\left(\varepsilon_{l}^{\prime}, 0\right)\left(\varepsilon_{l+1}^{\prime}, 0\right) \ldots\left(\varepsilon_{1}^{\prime}, 0\right)\left(\varepsilon_{2}^{\prime}, \varepsilon_{2}\right) \ldots$ é um caminho no autômato começando no estado inicial.

Portanto, usando o autômato temos:

$$
r=(1,0)(\varepsilon, \varepsilon+1)(a-1,0) \ldots
$$

ou

$$
r=(1,0)(\varepsilon, \varepsilon)(a-1,0)\left(\varepsilon_{1}^{\prime}+a-2, \varepsilon_{1}^{\prime}\right)\left(\varepsilon_{2}^{\prime \prime}, \varepsilon_{2}^{\prime \prime}\right)(0, a-1) \ldots
$$

ou

$$
r=(1,0)(\varepsilon, \varepsilon)(a-1,0)\left(\varepsilon^{\prime}+a-2, \varepsilon^{\prime}\right)\left(\varepsilon^{\prime \prime}, \varepsilon^{\prime \prime}+1\right) \ldots
$$

ou

$r=(1,0)(\varepsilon, \varepsilon)\left(\varepsilon^{\prime}+a-2, \varepsilon^{\prime}\right) w w w$ onde $w=(a-1,0)(0, a-1)(0, a-1)(a-1,0) \ldots$

Caso 1 Se $r=(1,0)(\varepsilon, \varepsilon+1)(a-1,0) \ldots$ então pelas equações (3.14) e (3.15) temos

$$
z=(\varepsilon+1) \alpha^{2}+\sum_{i=4}^{\infty} \varepsilon_{i} \alpha^{i}=\alpha+\varepsilon \alpha^{2}+(a-1) \alpha^{3}+\sum_{i=4}^{\infty} \varepsilon_{i}^{\prime} \alpha^{i} .
$$

Assim, $z \in \mathcal{R} \cap(\mathcal{R}+\alpha)$.

Caso 2 Se $r=(1,0)(\varepsilon, \varepsilon)(a-1,0)\left(\varepsilon_{1}^{\prime}+a-2, \varepsilon_{1}^{\prime}\right)\left(\varepsilon_{2}^{\prime \prime}, \varepsilon_{2}^{\prime \prime}\right)(0, a-1) \ldots$ Vamos então analisar as possibilidades:

Caso 2.1 Se $\varepsilon>0$ então temos

$$
z=\alpha+\varepsilon \alpha^{2}+(a-1) \alpha^{3}+\sum_{i=4}^{\infty} \varepsilon_{i}^{\prime} \alpha^{i}=\varepsilon \alpha^{2}+\sum_{i=4}^{\infty} \varepsilon_{i} \alpha^{i} .
$$

Portanto, $z \in \mathcal{R} \cap(\mathcal{R}+\alpha)$.

Caso 2.2 Se $\varepsilon=0$ e $\varepsilon_{1}^{\prime}>0$ então pelas equações (3.14) e (3.15) temos $\varepsilon_{1}=\varepsilon^{\prime}$, logo

$$
\begin{aligned}
z & =\varepsilon^{\prime} \alpha^{2}+\varepsilon_{2}^{\prime \prime} \alpha^{3}+(a-1) \alpha^{4}+\sum_{i=5}^{\infty} \varepsilon_{i} \alpha^{i} \\
& =\frac{1}{\alpha}+(a-1) \alpha+\left(\varepsilon_{1}^{\prime}+a-2\right) \alpha^{2}+\sum_{i=3}^{\infty} \varepsilon_{i}^{\prime} \alpha^{i}
\end{aligned}
$$


Como $\alpha^{3}=a \alpha^{2}-\alpha+1$ então $\frac{1}{\alpha}=\alpha^{2}-a \alpha+1$, assim

$$
\begin{array}{rlr}
z & = & \alpha^{2}-a \alpha+1+(a-1) \alpha+\left(\varepsilon_{1}^{\prime}+a-2\right) \alpha^{2}+\sum_{i=3}^{\infty} \varepsilon_{i}^{\prime} \alpha^{i} \\
& = & 1-\alpha+\left(\varepsilon_{1}^{\prime}+a-1\right) \alpha^{2}+\sum_{i=3}^{\infty} \varepsilon_{i}^{\prime} \alpha^{i}
\end{array}
$$

Portanto, $z \in \mathcal{R} \cap(\mathcal{R}-\alpha+1)$.

Caso 2.3 Se $\varepsilon=0, \varepsilon_{1}^{\prime}=0$ e $\varepsilon_{2}^{\prime \prime}>0$ então $\varepsilon_{2}^{\prime \prime}=\varepsilon_{2}$, logo das equaçôes (3.14) e (3.16) temos que

$$
\begin{aligned}
z & =\underbrace{\varepsilon_{2}^{\prime \prime} \alpha^{2}+(a-1) \alpha^{3}+\sum_{i=4}^{\infty} \varepsilon_{i} \alpha^{i}}_{\in \mathcal{R}} \\
& =\frac{1}{\alpha^{2}}+(a-1)+(a-2) \alpha+\varepsilon_{2}^{\prime \prime} \alpha^{2}+\sum_{i=3}^{\infty} \varepsilon_{i}^{\prime} \alpha^{i}
\end{aligned}
$$

Como $\alpha^{3}=a \alpha^{2}-\alpha+1$ então $\frac{1}{\alpha^{2}}=(1-a)+(1-a) \alpha+\alpha^{2}$, assim substituindo na segunda igualdade temos: $z=(1-a)+(1-a) \alpha+\alpha^{2}+(a-1)+(a-2) \alpha+\varepsilon_{2}^{\prime \prime} \alpha^{2}+\sum_{i=3}^{\infty} \varepsilon_{i}^{\prime} \alpha^{i}=$ $-\alpha+\left(\varepsilon_{2}^{\prime \prime}+1\right) \alpha^{2}+\sum_{i=3}^{\infty} \varepsilon_{i}^{\prime} \alpha^{i} \in \mathcal{R}+\alpha$.

Portanto, $z \in \mathcal{R} \cap(\mathcal{R}-\alpha)$.

Caso 2.4 Se $\varepsilon=0, \varepsilon_{1}^{\prime}=0, \varepsilon_{2}^{\prime \prime}=0$ então $\varepsilon_{2}=a-1$ e assim

$$
z=\underbrace{(a-1) \alpha^{2}+\sum_{i=3}^{\infty} \varepsilon_{i} \alpha^{i}}_{\in \mathcal{R}}=\frac{1}{\alpha^{3}}+\frac{(a-1)}{\alpha}+(a-2)+\sum_{i=2}^{\infty} \varepsilon_{i}^{\prime} \alpha^{i}
$$

Como $\frac{1}{\alpha^{3}}=(1-a)+\alpha+\frac{(1-a)}{\alpha}$, substituindo na segunda igualdade temos

$$
z=(1-a)+\alpha+\frac{(1-a)}{\alpha}+\frac{(a-1)}{\alpha}+(a-2)+\sum_{i=2}^{\infty} \varepsilon_{i}^{\prime} \alpha^{i}=-1+\alpha+\sum_{i=2}^{\infty} \varepsilon_{i}^{\prime} \alpha^{i} \in \mathcal{R}-1
$$

Portanto, $z \in \mathcal{R} \cap(\mathcal{R}+(\alpha-1))$.

Caso 3 Se $r=(1,0)(\varepsilon, \varepsilon)(a-1,0)\left(\varepsilon^{\prime}+a-2, \varepsilon^{\prime}\right)\left(\varepsilon^{\prime \prime}, \varepsilon^{\prime \prime}+1\right)$... então vamos analisar as possibilidades:

Caso 3.1 Se $\varepsilon>0$ então

$$
z=\alpha+\varepsilon \alpha^{2}+(a-1) \alpha^{3}+\sum_{i=4}^{\infty} \varepsilon_{i} \alpha^{i}=\varepsilon \alpha^{2}+\sum_{i=4}^{\infty} \varepsilon_{i} \alpha^{i}
$$

Assim, $z \in \mathcal{R} \cap(\mathcal{R}+\alpha)$ (resultado análogo ao caso 2.1). 
Caso 3.2 Se $\varepsilon=0, \varepsilon^{\prime}>0$ então $\varepsilon_{2}=\varepsilon^{\prime}$ e assim

$$
z=\frac{1}{\alpha}+(a-1) \alpha+\left(\varepsilon^{\prime}+a-2\right) \alpha^{2}+\sum_{i=3}^{\infty} \varepsilon_{i} \alpha^{i}=\underbrace{\varepsilon^{\prime} \alpha^{2}+\sum_{i=3}^{\infty} \varepsilon_{i}^{\prime} \alpha^{i}}_{\in \mathcal{R}}
$$

Se $\frac{1}{\alpha}=\alpha^{2}-a \alpha+1$ então substituindo temos:

$z=1-a \alpha+\alpha^{2}+(a-1) \alpha+\left(\varepsilon^{\prime}+a-2\right) \alpha^{2}+\sum_{i=3}^{\infty} \varepsilon_{i} \alpha^{i}=1-\alpha+\left(\varepsilon^{\prime}+a-1\right) \alpha^{2}+\sum_{i=3}^{\infty} \varepsilon_{i} \alpha^{i}=$

Portanto, $z \in \mathcal{R} \cap(\mathcal{R}+(1-\alpha))$.

Caso 3.3 Se $\varepsilon=0, \varepsilon^{\prime}=0 \operatorname{logo} \varepsilon_{2}=\varepsilon^{\prime \prime}+1>0$ então

$$
z=\underbrace{\left(\varepsilon^{\prime \prime}+1\right) \alpha^{3}+\sum_{i=3}^{\infty} \varepsilon_{i} \alpha^{i}}_{\in \mathcal{R}}=\varepsilon^{\prime \prime} \alpha^{2}+(a-2) \alpha+(a-1)+\frac{1}{\alpha^{2}}+\sum_{i=3}^{\infty} \varepsilon_{i} \alpha^{i}
$$

Se $\frac{1}{\alpha^{2}}=(1-a)+(1-a) \alpha+\alpha^{2}$ então substituindo na segunda igualdade temos:

$$
\begin{array}{lll}
z & = & (1-a)+(1-a) \alpha+\alpha^{2}+(a-1)+(a-2) \alpha+\varepsilon^{\prime \prime} \alpha^{2}+\sum_{i=3}^{\infty} \varepsilon_{i} \alpha^{i} \\
z & = & -\alpha+\left(\varepsilon^{\prime \prime}+1\right) \alpha^{2}+\sum_{i=3}^{\infty} \varepsilon_{i}^{\prime} \alpha^{i}=\left(\varepsilon^{\prime \prime}+1\right) \alpha^{2}+\sum_{i=3}^{\infty} \varepsilon_{i} \alpha^{i}
\end{array}
$$

Portanto, $z \in \mathcal{R} \cap(\mathcal{R}-\alpha)$.

Caso 4 Vamos considerar o caminho: $r=(1,0)(\varepsilon, \varepsilon)\left(\varepsilon^{\prime}+a-2, \varepsilon^{\prime}\right)$ www onde $w=(a-1,0)(0, a-1)(0, a-1)(a-1,0)$. Analisando os casos temos:

Caso 4.1 Se $\varepsilon>0$ então

$$
z=\alpha+\varepsilon \alpha^{2}+\left(\varepsilon^{\prime}+a-2\right) \alpha^{2}+\sum_{i=3}^{\infty} \varepsilon_{i} \alpha^{i}=\varepsilon \alpha^{2}+\varepsilon^{\prime} \alpha^{3}+\sum_{i=4}^{\infty} \varepsilon_{i} \alpha^{i}
$$

Logo, $z \in \mathcal{R} \cap(\mathcal{R}+\alpha)$.

Caso 4.2 Se $\varepsilon=0$ e $\varepsilon^{\prime}>0$ então $\varepsilon_{2}=\varepsilon^{\prime}$ então

$$
z=\varepsilon^{\prime} \alpha^{2}+\sum_{i=3}^{\infty} \varepsilon_{i} \alpha^{i}=1+\left(\varepsilon^{\prime}+a-2\right) \alpha^{2}+\sum_{i=3}^{\infty} \varepsilon_{i} \alpha^{i} .
$$

Portanto, $z \in \mathcal{R} \cap(\mathcal{R}+1)$. 
Caso 4.3 Se $\varepsilon=0, \varepsilon^{\prime}=0$ então $\varepsilon_{2}=a-1, \operatorname{logo}$

$$
z=\underbrace{(a-1) \alpha^{2}+\sum_{i=3}^{\infty} \varepsilon_{i} \alpha^{i}}_{\in \mathcal{R}}=\frac{1}{\alpha^{2}}+(a-2)+(a-1) \alpha+\sum_{i=4}^{\infty} \varepsilon_{i}^{\prime} \alpha^{i} \text { pois } \varepsilon_{2}^{\prime}=\varepsilon_{3}^{\prime}=0 .
$$

Como $\alpha^{-2}=(1-a)+(1-a) \alpha+\alpha^{2}$ então substituindo na segunda igualdade temos:

$$
z=(1-a)+(1-a) \alpha+\alpha^{2}+(a-2)+(a-1) \alpha+\sum_{i=4}^{\infty} \varepsilon_{i} \alpha^{i}=-1+\alpha^{2}+\sum_{i=4}^{\infty} \varepsilon_{i}^{\prime} \alpha^{i} .
$$

Portanto, $z \in \mathcal{R} \cap(\mathcal{R}-1)$.

Conjectura: Pelo Teorema (2.2.1) sabemos que no caso $a=2, \mathcal{R}$ possui exatamente 6 vizinhos tais que $(\mathcal{R}+u), \pm u \in B$. Conjecturamos que o mesmo acontece para todo a $>2$. 

Capítulo

\section{Aplicação do autômato para a}

\section{parametrização da Fronteira de $\mathcal{R}$}

Usando o autômato, provamos explicitamente para o caso $a=2$, que cada uma das 6 regióes $\mathcal{R} \cap(\mathcal{R}+u)=\mathcal{R}_{u}, u \in B$, que constitui a fronteira de $\mathcal{R}$ é a imagem de uma outra por uma aplicação afim. Mostraremos também que se $\mathcal{R}_{u}$ é uma das 6 regiões, então existem 3 funções afins $g_{0}, g_{1}, g_{2}$ tais que $\mathcal{R}_{u}=\bigcup_{i=0,1,2} g_{i}\left(\mathcal{R}_{u}\right)$. Com isso construiremos uma aplicação contínua e bijetora de $[0,1]$ para $\mathcal{R}_{u}$. Como consequência teremos que a fronteira de $\mathcal{R}$ é homeomorfa ao círculo e também calcularemos explicitamente a dimensão de Hausdorff da $\partial \mathcal{R}$.

Por simplicidade, supomos em todo esse capítulo que $a=2$. Sabemos que nesse caso, pelo Teorema 3.2.3, que $\partial \mathcal{R}$ é formada por 6 regiões $\mathcal{R} \cap(\mathcal{R}+u)$, onde $u \in B=\{ \pm 1, \pm \alpha, \pm(\alpha-1)\}$.

Lema 4.0.4 As propriedades seguintes são válidas:

1. $\mathcal{R}_{\alpha-1} \cap \mathcal{R}_{\alpha}=\{w\}=\left\{\alpha^{2}-\alpha^{3}\right\}$;

2. $\mathcal{R}_{\alpha} \cap \mathcal{R}_{1}=\left\{-\alpha^{3}\right\}$;

3. $\mathcal{R}_{\alpha-1} \cap \mathcal{R}_{-1}=\left\{-\alpha^{3}-1\right\}=\{u\}$;

4. $\mathcal{R}_{-(\alpha-1)} \cap \mathcal{R}_{-\alpha}=\left\{-\alpha^{3}-\alpha\right\}$

5. $\mathcal{R}_{-(\alpha-1)} \cap \mathcal{R}_{1}=\{w+1-\alpha\}$;

6. $\mathcal{R}_{-\alpha} \cap \mathcal{R}_{-1}=\{w-\alpha\}$. 


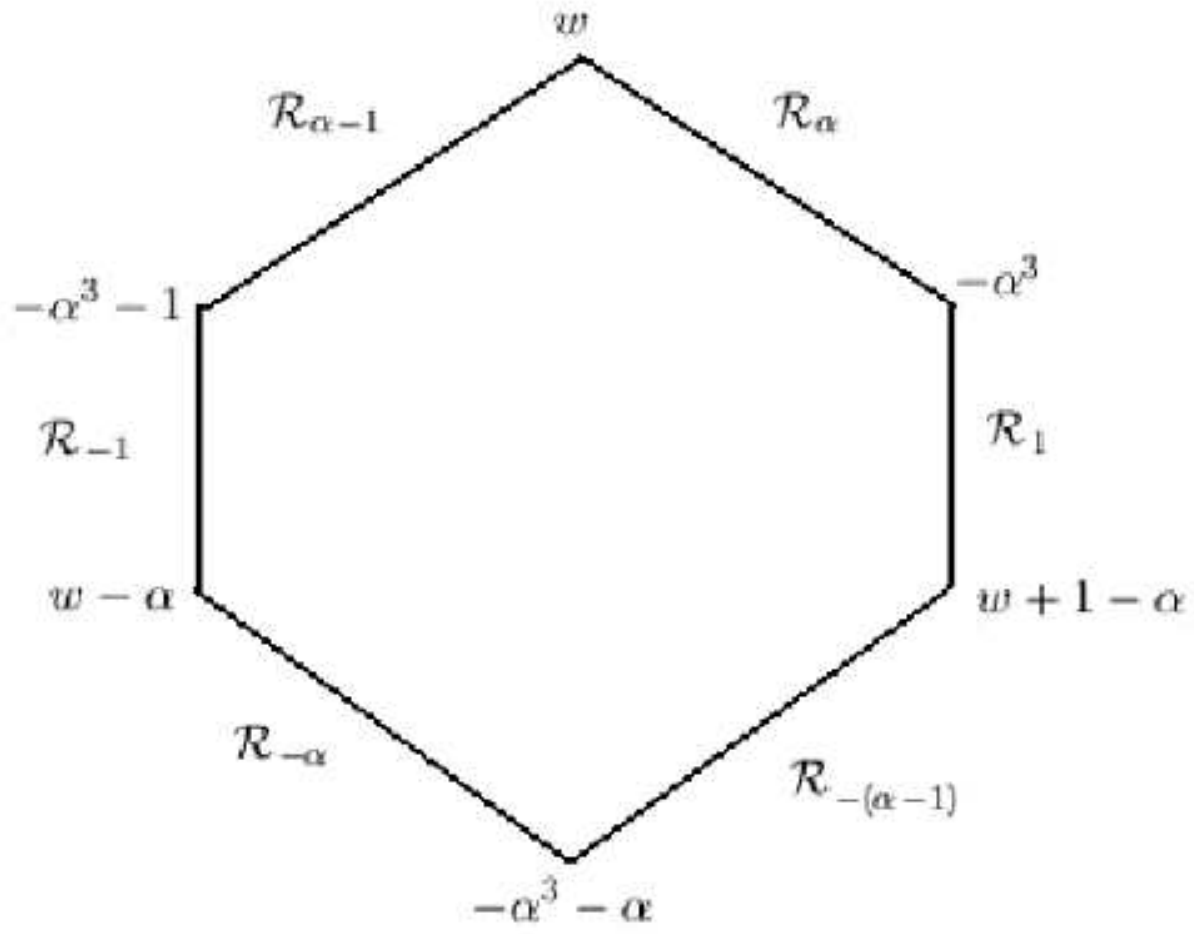

Demonstração: Seja w um elemento de $\mathcal{R}_{\alpha-1} \cap \mathcal{R}_{\alpha}=\mathcal{R} \cap(\mathcal{R}+\alpha-1) \cap(\mathcal{R}+\alpha)$ então existem $x$, y e $z \in \mathcal{R}$ tais que $x=\sum_{i=2}^{+\infty} a_{i} \alpha^{i}, y=\sum_{i=2}^{+\infty} b_{i} \alpha^{i}$ e $z=\sum_{i=2}^{+\infty} c_{i} \alpha^{i}$ e

$$
w=\alpha-1+x=\alpha+y=z
$$

Por outro lado, pela Proposição 1.1.2, $d(1, \beta)=(a-1)(a-1) 01$ logo $1=\beta^{-1}+\beta^{-2}+\beta^{-4}$, então $\beta=1+\beta^{-1}+\beta^{-3}$, portanto,

$$
\alpha-1=\alpha^{-3}+\alpha^{-1}
$$

Usando as equaçôes (4.1) e (4.2) e o autômato obtemos $r_{1}=(1,0)(0,0)(1,0)(0,0)(0,0)\left(a_{2}, c_{2}\right)\left(a_{3}, c_{3}\right) \ldots$ e $r_{2}=(1,0)(0,0)(1,0)(0,0)(0,1)\left(a_{2}, b_{2}\right)\left(a_{3}, b_{3}\right) \ldots$ são caminhos do autômato que começam no estado inicial.

Analisando o autômato obtemos

$$
\begin{array}{lcc}
r_{1}= & (1,0)(0,0)(1,0)(0,0)(0,0)(0,1)\left(a_{3}, c_{3}\right) \ldots e \\
r_{2}= & (1,0)(0,0)(1,0)(0,0)(0,1)(0,1)(0,0)\left(a_{4}, b_{4}\right) \text { ou } \\
r_{2}= & (1,0)(0,0)(1,0)(0,0)(0,1)(0,0) \text { ttttt onde } t=(0,1)(1,0)(1,0)(0,1) .
\end{array}
$$

Supomos o primeiro caso: $r_{1}=(1,0)(0,0)(1,0)(0,0)(0,0)(0,1)\left(a_{3}, c_{3}\right) \ldots e$ 
$r_{2}=(1,0)(0,0)(1,0)(0,0)(0,1)(0,1)(0,0)\left(a_{4}, b_{4}\right) \ldots$ e analisando o autômato temos que o estado entre $(0,1)$ e $\left(a_{3}, c_{3}\right)$ é igual $a-\alpha-1 / \alpha=-1+\alpha-\alpha^{2}$ é o mesmo estado entre $(0,0)$ e $\left(a_{4}, b_{4}\right)$.

Logo, utilizando o autômato temos:

$$
\begin{aligned}
& r_{1}=(1,0)(0,0)(1,0)(0,0)(0,0)(0,1)(0,0)(1,0)(1,1)(0,1)(0,0)\left(a_{8}, c_{8}\right) \ldots e \\
& r_{2}=(1,0)(0,0)(1,0)(0,0)(0,1)(0,1)(0,0)(1,1)(1,0)(0,0)(0,1)\left(a_{8}, b_{8}\right) \ldots
\end{aligned}
$$

Continuando assim obtemos:

$$
\begin{aligned}
& r_{1}=\quad(1,0)(0,0)(1,0)(0,0)(0,0)(0,1)(0,0)(1,0)(1,1)(0,1)(0,0)(1,0)(1,1)(0,1)(0,0) \ldots e \\
& r_{2}=(1,0)(0,0)(1,0)(0,0)(0,1)(0,1)(0,0)(1,1)(1,0)(0,0)(0,1)(1,1)(1,0)(0,0)(0,1)(1,1)(1,0)(0,0)(0,1) \ldots
\end{aligned}
$$

Assim

$$
w=\alpha-1+x=\alpha^{-3}+\alpha^{-1}+\sum_{i=1}^{+\infty}\left(\alpha^{4 i}+\alpha^{4 i+1}\right)
$$

Por outro lado

$$
w=z=\alpha^{2}+\sum_{i=1}^{+\infty}\left(\alpha^{4 i+1}+\alpha^{4 i+2}\right)
$$

$e$

$$
w=\alpha+y=\alpha+\alpha^{2}+\alpha^{4}+\sum_{i=2}^{+\infty}\left(\alpha^{4 i+3}+\alpha^{4 i+4}\right)
$$

Supondo o segundo caso: $r_{1}=(1,0)(0,0)(1,0)(0,0)(0,0)(0,1)\left(a_{3}, c_{3}\right) \ldots e$ $r_{2}=(1,0)(0,0)(1,0)(0,0)(0,1)(0,0)$ ttttt $\ldots$ onde $t=(0,1)(1,0)(1,0)(0,1)$ obtemos

$$
w=\alpha-1+x=\alpha^{-3}+\alpha^{-1}+\sum_{i=1}^{+\infty}\left(\alpha^{4 i}+\alpha^{4 i+1}\right)
$$

Por outro lado

$$
w=z=\alpha^{2}+\sum_{i=1}^{+\infty}\left(\alpha^{4 i+1}+\alpha^{4 i+2}\right)
$$

$e$

$$
w=\alpha+y=\alpha+\alpha^{3}+\sum_{i=2}^{+\infty}\left(\alpha^{4 i+2}+\alpha^{4 i+3}\right)
$$

Das equaçôes (4.3) à (4.8) obtemos, por exemplo (usando a equação (4.4)):

$$
w=\alpha^{2}+\left(\alpha^{5}+\alpha^{6}\right)+\left(\alpha^{9}+\alpha^{10}\right)+\left(\alpha^{13}+\alpha^{14}\right)+\ldots
$$

Como $\alpha^{7}=\alpha^{6}+\alpha^{5}+\alpha^{3}$ então

$$
w=\alpha^{2}+\left(\alpha^{7}-\alpha^{3}\right)+\left(\alpha^{11}-\alpha^{7}\right)+\left(\alpha^{15}-\alpha^{11}\right)+\ldots
$$

Portanto, $w=\alpha^{2}-\alpha^{3}$. 
Da mesma maneira provamos $\mathcal{R}_{\alpha} \cap \mathcal{R}_{1}=\left\{-\alpha^{3}\right\}$. As outras propriedades decorrem facilmente dos itens 1 e 2.

Vamos mostrar que $\mathcal{R}_{\alpha-1}$ se decompõe em três subconjuntos, que são as imagens de $\mathcal{R}_{\alpha-1}$ por cada uma das três funçôes $g_{i}, i \in\{0,1,2\}$ definidas por:

$$
g_{0}(z)=\alpha^{-3}+\alpha^{-1}+\alpha^{2} z, \quad g_{1}(z)=-1-\alpha^{3}+\alpha^{3} z, \quad \text { e } g_{2}(z)=\alpha^{2}+\alpha^{5}+\alpha^{4} z, \quad z \in \mathbb{C}
$$

De fato, mostraremos que $\mathcal{R}_{\alpha-1}$ é homeomorfo a um segmento de reta. Sejam $a, b \in \mathcal{R}_{\alpha-1}$. Se denotarmos por $I(a, b)$ o segmento em $\mathcal{R}_{\alpha-1}$ ligando a e $b$, em virtude do Lema 4.0.5 veremos que

$$
\begin{aligned}
& g_{0}\left(\mathcal{R}_{\alpha-1}\right)=I\left(-\alpha^{3}-1,-\left(\alpha^{-1}+\alpha+\alpha^{5}\right)\right)=I\left(g_{0}\left(\alpha^{2}-\alpha^{3}\right), g_{0}\left(-\alpha^{3}-1\right)\right)=I\left(g_{0}(w), g_{0}(u)\right) \\
& g_{1}\left(\mathcal{R}_{\alpha-1}\right)=I\left(-\left(\alpha^{-1}+\alpha+\alpha^{5}\right),-\left(\alpha^{-1}+\alpha+\alpha^{4}+\alpha^{6}\right)\right)=I\left(g_{1}\left(\alpha^{2}-\alpha^{3}\right), g_{1}\left(-\alpha^{3}-1\right)\right)=I\left(g_{1}(w), g_{1}(u)\right) \\
& g_{2}\left(\mathcal{R}_{\alpha-1}\right)=I\left(-\left(\alpha^{-1}+\alpha+\alpha^{4}+\alpha^{6}\right), \alpha^{2}-\alpha^{3}\right)=I\left(g_{2}\left(-\alpha^{3}-1\right), g_{2}\left(\alpha^{2}-\alpha^{3}\right)\right)=I\left(g_{2}(u), g_{2}(w)\right) .
\end{aligned}
$$




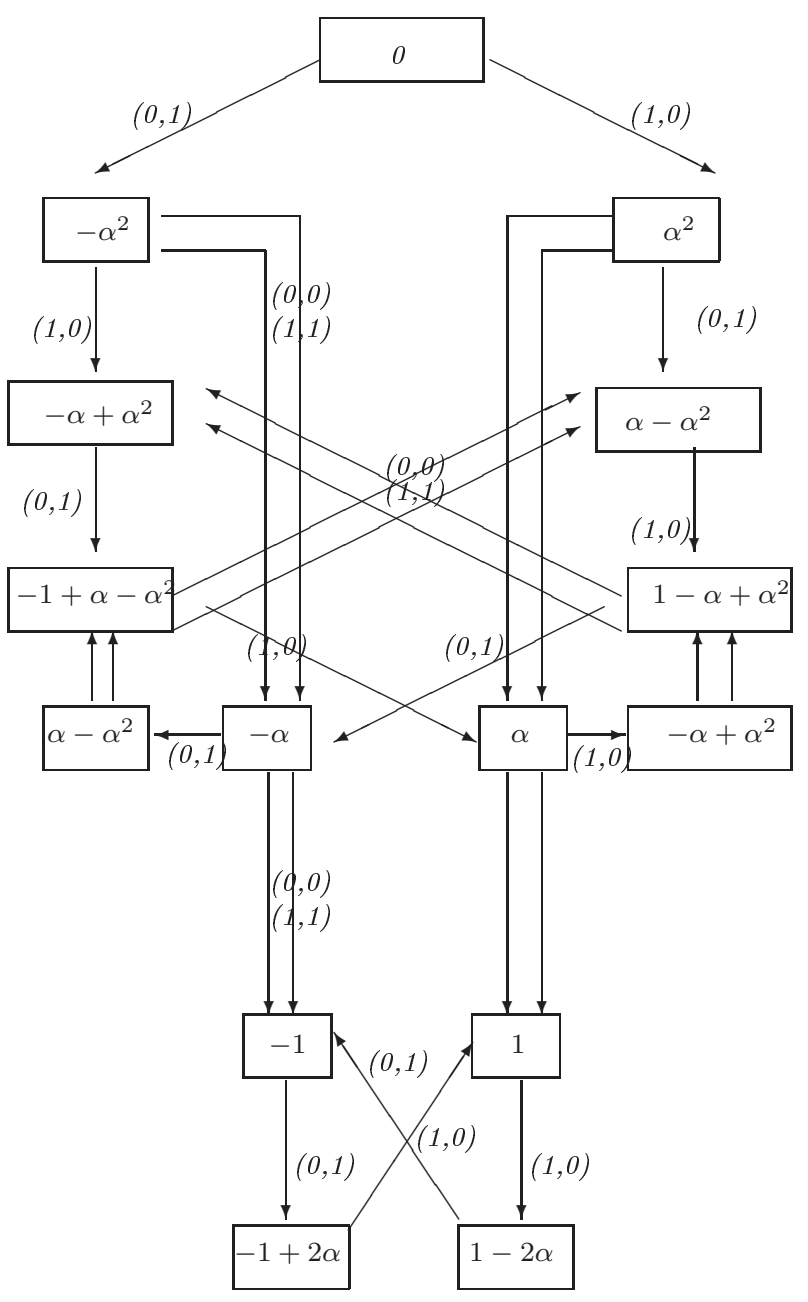

Autômato para o Caso $a=2$. 


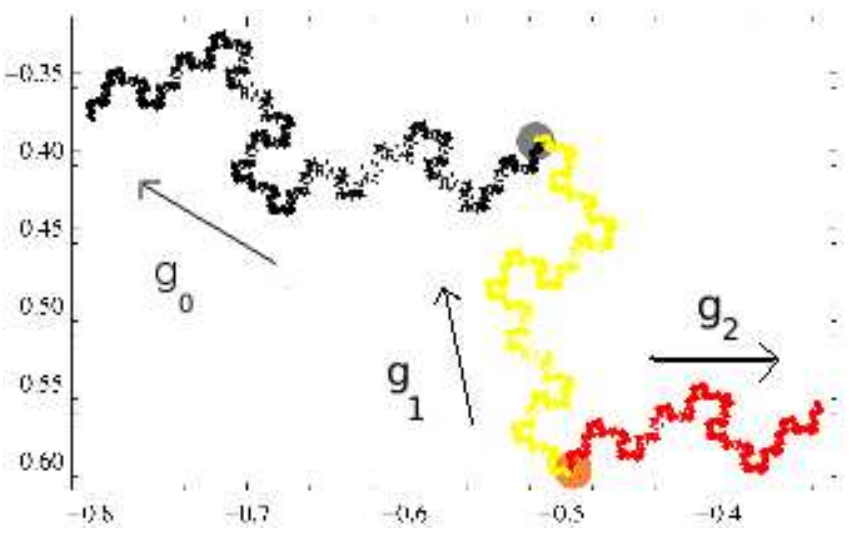

Lema 4.0.5 $\mathcal{R}_{\alpha-1}$ verifica as seguintes propriedades, onde $u=-1-\alpha^{3}$ e $w=\alpha^{2}-\alpha^{3}$ :

1. $\mathcal{R}_{\alpha-1}=g_{0}\left(\mathcal{R}_{\alpha-1}\right) \cup g_{1}\left(\mathcal{R}_{\alpha-1}\right) \cup g_{2}\left(\mathcal{R}_{\alpha-1}\right)$;

2. $g_{0}\left(\mathcal{R}_{\alpha-1}\right) \cap g_{1}\left(\mathcal{R}_{\alpha-1}\right)=\left\{-\left(\alpha^{-1}+\alpha+\alpha^{5}\right)\right\}=\left\{g_{0}(u)\right\}=\left\{g_{1}(w)\right\}$;

3. $g_{1}\left(\mathcal{R}_{\alpha-1}\right) \cap g_{2}\left(\mathcal{R}_{\alpha-1}\right)=\left\{-\left(\alpha^{-1}+\alpha+\alpha^{4}+\alpha^{6}\right)\right\}=\left\{g_{1}(u)\right\}=\left\{g_{2}(u)\right\}$;

4. $g_{0}\left(\mathcal{R}_{\alpha-1}\right) \cap g_{2}\left(\mathcal{R}_{\alpha-1}\right)=\emptyset$.

\section{Demonstração:}

1. Vamos mostrar que $g_{i}\left(\mathcal{R}_{\alpha-1}\right) \subset \mathcal{R}_{\alpha-1}, \forall i \in\{0,1,2\}$.

$g_{0}\left(\mathcal{R}_{\alpha-1}\right)=\left(\alpha^{-3}+\alpha^{-1}+\alpha^{2} \mathcal{R}\right) \cap\left(\alpha^{-3}+\alpha^{-1}+\alpha+\alpha^{-1}+\alpha^{2} \mathcal{R}\right)=\left(\alpha^{-3}+\alpha^{-1}+\alpha^{2} \mathcal{R}\right) \cap\left(\alpha^{2}+\right.$ $\left.\alpha^{2} \mathcal{R}\right)=\left((\alpha-1)+\alpha^{2} \mathcal{R}\right) \cap\left(\alpha^{2}+\alpha^{2} \mathcal{R}\right)$ e $g_{2}\left(\mathcal{R}_{\alpha-1}\right)=\left(\alpha^{2}+\alpha^{5}+\alpha^{4} \mathcal{R}\right) \cap\left(\alpha^{2}+2 \alpha^{5}-\alpha^{4}+\alpha^{4} \mathcal{R}\right)$.

Por outro lado $\alpha^{2}+2 \alpha^{5}-\alpha^{4}=\alpha^{2}+\alpha^{5}+\alpha^{3}-\alpha=(\alpha-1)+1+\alpha^{2}+\alpha^{3}+\alpha^{5}=(\alpha-1)+\alpha^{4}+\alpha^{5}$.

Logo, $g_{2}\left(\mathcal{R}_{\alpha-1}\right)=\left(\alpha^{2}+\alpha^{5}+\alpha^{4} \mathcal{R}\right) \cap\left((\alpha-1)+\alpha^{4}+\alpha^{5}+\alpha^{4} \mathcal{R}\right) \subset \mathcal{R}_{\alpha-1}$.

Seja $x \in \mathcal{R}_{\alpha-1}$ então $x=\alpha-1+\sum_{i=2}^{\infty} \varepsilon_{i} \alpha^{i}=\sum_{i=2}^{\infty} \varepsilon_{i}^{\prime} \alpha^{i}$ onde $\left(\varepsilon_{i}\right)_{i \geq 2},\left(\varepsilon_{i}^{\prime}\right)_{i \geq 2} \in \mathcal{D}^{\infty}$.

Como $\alpha-1=\alpha^{-3}+\alpha^{-1}$ então

$$
(1,0)(0,0)(1,0)(0,0)(0,0)\left(\varepsilon_{2}, \varepsilon_{2}^{\prime}\right)\left(\varepsilon_{3}, \varepsilon_{3}^{\prime}\right)\left(\varepsilon_{4}, \varepsilon_{4}^{\prime}\right) \ldots
$$

é um caminho no autômato começando no estado inicial.

Portanto, a única possibilidade é $\left(\varepsilon_{2}, \varepsilon_{2}^{\prime}\right)\left(\varepsilon_{3}, \varepsilon_{3}^{\prime}\right)\left(\varepsilon_{4}, \varepsilon_{4}^{\prime}\right)=(0,1)(0,0)(1,0)$.

Logo $x=\alpha^{-3}+\alpha^{-1}+\alpha^{4}+\sum_{i=5}^{\infty} \varepsilon_{i} \alpha^{i}=\alpha^{2}+\sum_{i=5}^{\infty} \varepsilon_{i}^{\prime} \alpha^{i}$.

Assim,

$$
x=\alpha^{-3}+\alpha^{-1}+\alpha^{4}+\alpha^{3} z=\alpha^{2}+\alpha^{3} z^{\prime}, \quad \text { onde } z, z^{\prime} \in \mathcal{R}
$$


Então, $g_{1}(x)=-1-\alpha^{3}+\alpha^{3}\left(\alpha^{2}+\alpha^{3} z^{\prime}\right)$ e $g_{1}(x)=-1-\alpha^{3}+\alpha^{3}\left(\alpha^{-3}+\alpha^{-1}+\alpha^{4}+\alpha^{3} z\right)$.

Logo $g_{1}(x)=-1-\alpha^{3}+\alpha^{5}+\alpha^{6} z=-1+\alpha+\alpha^{4}+\alpha^{6} z \in(\mathcal{R}+\alpha-1)$ e $g_{1}(x)=-\alpha^{3}+\alpha^{2}+$ $\alpha^{7}+\alpha^{6} z^{\prime}=\alpha^{2}+\alpha^{5}+\alpha^{6}+\alpha^{6} z^{\prime} \in \mathcal{R}$.

Portanto, $g_{1}(x) \in \mathcal{R}_{\alpha-1}$, logo $g_{1}\left(\mathcal{R}_{\alpha-1}\right) \subset \mathcal{R}_{\alpha-1}$.

Agora vamos mostrar a inclusão inversa.

Seja z um elemento de $\mathcal{R}_{\alpha-1}$. Então

$$
z=\alpha^{-3}+\alpha^{-1}+\alpha^{4}+\sum_{i=5}^{\infty} \varepsilon_{i} \alpha^{i}=\alpha^{2}+\sum_{i=5}^{\infty} \varepsilon_{i}^{\prime} \alpha^{i}, \quad \text { onde }\left(\varepsilon_{i}\right)_{i \geq 5}, \quad\left(\varepsilon_{i}^{\prime}\right)_{i \geq 5} \in \mathcal{D}^{\infty}
$$

$e(1,0)(0,0)(1,0)(0,0)(0,0)(0,1)(0,0)(1,0)\left(\varepsilon_{5}, \varepsilon_{5}^{\prime}\right) \ldots$ é um caminho no autômato.

Em virtude do autômato dos complexos duplos, temos $z=\frac{1}{\alpha^{3}}+\frac{1}{\alpha}+\alpha^{2} w=\alpha^{2}+\alpha^{2} w^{\prime}$, onde $w, w^{\prime} \in \mathcal{R}$ pois $(1,0)(0,0)(1,0)(0,0)(0,0)(0,1) \ldots$ é o único caminho em $\mathcal{A}$ que começa com $(1,0),(0,0)(1,0)$. Assim temos as possibilidades:

Analisando o autômato temos as seguintes três possibilidades: $\left(\varepsilon_{5}, \varepsilon_{5}^{\prime}\right)\left(\varepsilon_{6}, \varepsilon_{6}^{\prime}\right)=(0,0)(0,1)$ ou $\left(\varepsilon_{5}, \varepsilon_{5}^{\prime}\right)\left(\varepsilon_{6}, \varepsilon_{6}^{\prime}\right)=(1,1)(0,1)$ ou $\left(\varepsilon_{5}, \varepsilon_{5}^{\prime}\right)\left(\varepsilon_{6}, \varepsilon_{6}^{\prime}\right)=(0,1)(0,1)$.

- $\quad S e\left(\varepsilon_{5}, \varepsilon_{5}^{\prime}\right)\left(\varepsilon_{6}, \varepsilon_{6}^{\prime}\right)=(0,0)(0,1)$

então neste caso temos $z=\alpha^{-3}+\alpha^{-1}+\alpha^{2} w_{0}=\alpha^{2}+\alpha^{2} w_{0}^{\prime}$ onde $w_{0}, w_{0}^{\prime} \in \mathcal{R}$. Logo $g_{0}^{-1}(z) \in$ $\mathcal{R}_{\alpha-1}$, portanto, $z \in g_{0}\left(\mathcal{R}_{\alpha-1}\right)$.

- $S e\left(\varepsilon_{5}, \varepsilon_{5}^{\prime}\right)\left(\varepsilon_{6}, \varepsilon_{6}^{\prime}\right)=(0,1)(0,1)$ então $z=\underbrace{\alpha^{-3}+\alpha^{-1}}_{\alpha-1}+\alpha^{4}+\sum_{i=7} \varepsilon_{i} \alpha^{i}=\alpha^{2}+\alpha^{5}+\alpha^{6}+$ $\sum_{i=8}^{\infty} \varepsilon_{i}^{\prime} \alpha^{i}$

Logo $g_{1}^{-1}(z)=\alpha^{-3}+1+\alpha^{-2}-\alpha^{-3}+\alpha+\sum_{i=7}^{\infty} \varepsilon_{i} \alpha^{i-3}=\alpha^{-3}+1+\alpha^{-1}+\alpha^{2}+\alpha^{3}+\sum_{i=8}^{\infty} \varepsilon_{i}^{\prime} \alpha^{i-3}$. Assim, $g^{-1}(z)=\alpha^{-2}+1+\alpha+\sum_{i=7}^{\infty} \varepsilon_{i} \alpha^{i-3}=\alpha^{-3}+\alpha^{-1}+1+\alpha^{2}+\alpha^{3}+\sum_{i=8}^{\infty} \varepsilon_{i} \alpha^{i-3}$.

Então, $g_{1}^{-1}(z)=\alpha^{2}+\sum_{i=7}^{\infty} \varepsilon_{i} \alpha^{i-3}=(\alpha-1)+\alpha^{4}+\sum_{i=8}^{\infty} \varepsilon_{i}^{\prime} \alpha^{i-3}$.

Portanto, $g_{1}^{-1}(z) \in \mathcal{R}_{\alpha-1}$, logo $z \in g_{1}\left(\mathcal{R}_{\alpha-1}\right)$.

- $\operatorname{Se}\left(\varepsilon_{5}, \varepsilon_{5}^{\prime}\right)\left(\varepsilon_{6}, \varepsilon_{6}^{\prime}\right)=(1,1)(0,1)$ então $z=\underbrace{\alpha^{-3}+\alpha^{-1}}_{\alpha-1}+\alpha^{4}+\sum_{i=7}^{\infty} \varepsilon_{i} \alpha^{i}=\alpha^{2}+\alpha^{5}+\alpha^{6}+$ $\sum_{i=8}^{\infty} \varepsilon_{i}^{\prime} \alpha^{i}$

Logo $g_{2}^{-1}(z)=-\alpha^{-2}-\alpha+\alpha^{-3}-\alpha^{-4}+1+\alpha+\sum_{i=7}^{\infty} \varepsilon_{i} \alpha^{i-4}=-\alpha^{-2}-\alpha+\alpha^{-2}+\alpha+\alpha^{2}+$ $\sum_{i=8}^{\infty} \varepsilon_{i}^{\prime} \alpha^{i-4}$.

Ent $\tilde{a} o, g_{2}^{-1}(z)=-\alpha^{-2}+\alpha^{-3}-\alpha^{-4}+\alpha^{-1}+\alpha^{-2}+\alpha^{-4}+\sum_{i=7}^{\infty} \varepsilon_{i} \alpha^{i-4}=\alpha^{2}+\sum_{i=8}^{\infty} \varepsilon_{i}^{\prime} \alpha^{i-4}$. 
Assim, $g_{2}^{-1}(z)=\underbrace{\alpha^{-3}+\alpha^{-1}}_{\alpha-1}+\sum_{i=7}^{\infty} \varepsilon_{i} \alpha^{i-4}=\alpha^{2}+\sum_{i=8}^{\infty} \varepsilon_{i}^{\prime} \alpha^{i-4}$.

Portanto, $g_{2}^{-1}\left(\mathcal{R}_{\alpha-1}\right) \subset \mathcal{R}_{\alpha-1}$, logo $z \in g_{2}\left(\mathcal{R}_{\alpha-1}\right)$.

2. Suponha que $\gamma \in g_{0}\left(\mathcal{R}_{\alpha-1}\right) \cap g_{1}\left(\mathcal{R}_{\alpha-1}\right)$.

Então existem $y, z \in \mathcal{R}_{\alpha-1}$ tais que $\gamma=g_{0}(y)=g_{1}(z)$ isto é

$$
\underbrace{\alpha^{-1}+\alpha^{-3}}_{\alpha-1}+\alpha^{2} y=-1-\alpha^{3}+\alpha^{3} z
$$

Logo, $y=-\alpha^{-1}-\alpha+\alpha z$. Por (4.9) podemos escrever os elementos de $\mathcal{R}_{\alpha-1}$ de duas maneiras. Considerando a primeira $z=\alpha-1+\alpha^{4}+\alpha^{3} \widetilde{z}, \widetilde{z} \in \mathcal{R}$, e como $\alpha^{2}-2 \alpha+1-\alpha^{-1}=0$ substituindo em (4.10) temos:

$$
\begin{array}{rlrl}
y & = & -\alpha^{-1}-\alpha+\alpha\left(\alpha-1+\alpha^{4}+\alpha^{3} \widetilde{z}\right) \\
& = & -\alpha^{-1}-\alpha+\alpha^{2}-\alpha+\alpha^{5}+\alpha^{5} \widetilde{z} \\
& = & & \left(-2 \alpha-\alpha^{-1}\right)+\alpha^{2}+\alpha^{5}+\alpha^{4} \widetilde{z} \\
& = & -\alpha^{2}-1+\alpha^{2}+\alpha^{5}+\alpha^{4} \widetilde{z} \\
& = & -1+\alpha^{5}+\alpha^{4} \widetilde{z} \in(\mathcal{R}-1)
\end{array}
$$

Portanto, $y \in \mathcal{R} \cap(\mathcal{R}+\alpha-1) \cap(\mathcal{R}-1)$.

Agora considerando a segunda maneira $z=\alpha^{2}+\alpha^{3} \widehat{z}, \widehat{z} \in \mathcal{R}$ e como $\alpha^{3}=\alpha^{2}+\alpha+1 / \alpha$, substituindo em (4.10) temos

$$
\begin{array}{rlc}
y & = & -\alpha^{-1}-\alpha+\alpha\left(\alpha^{2}+\alpha^{3} \widehat{z}\right) \\
& = & -\alpha^{-1}-\alpha+\alpha^{3}+\alpha^{4} \widehat{z} \\
& = & -\alpha^{-1}-\alpha+\alpha^{2}+\alpha+\frac{1}{\alpha}+\alpha^{4} \widehat{z} \\
& = & \alpha^{2}+\alpha^{4} \widehat{z}
\end{array}
$$

Dos dois casos anteriores segue que $y \in \mathcal{R} \cap(\mathcal{R}+\alpha-1) \cap(\mathcal{R}-1)$ e pelo Lema (4.0.4), $y=-1-\alpha^{3}=u$. Assim, $y=g_{0}(u)=g_{1}(w)$.

Portanto, $g_{0}\left(\mathcal{R}_{\alpha-1}\right) \cap g_{1}\left(\mathcal{R}_{\alpha-1}\right)=\left\{-\left(\alpha^{-1}+\alpha+\alpha^{5}\right)\right\}=g_{0}(u)=g_{1}(w)$.

3. Suponha $\gamma \in g_{1}\left(\mathcal{R}_{\alpha-1}\right) \cap g_{2}\left(\mathcal{R}_{\alpha-1}\right)$.

Então existem $y, z \in \mathcal{R}_{\alpha-1}$ tais que $\gamma=g_{1}(y)=g_{2}(z)$ logo $\gamma=-1-\alpha^{-3}+\alpha^{3} y=\alpha^{2}+\alpha^{5}+\alpha^{4} z$. Desta forma,

$$
y=\alpha^{-3}+\alpha^{-1}+1+\alpha^{2}+\alpha z .
$$

Pela equação (4.9) temos que os elementos de $\mathcal{R}_{\alpha-1}$ podem ser escritos de duas maneiras.

Considerando $y=\alpha^{2}+\alpha^{3} \widetilde{z}, \widetilde{z} \in \mathcal{R}$, temos, $z=-1-\alpha+\frac{1}{\alpha}\left(\alpha^{2}+\alpha^{3} \widehat{z}\right)=-1+\alpha^{2} \widehat{z} \in \mathcal{R}-1$. 
Da análise acima temos que $z \in \mathcal{R} \cap(\mathcal{R}+\alpha-1) \cap(\mathcal{R}-1)$ e, portanto, pelo Lema (4.0.4), $z=-1-\alpha^{3}$. Assim,

$$
\begin{array}{rcc}
\gamma & = & \alpha^{2}+\alpha^{5}+\alpha^{4} z=\alpha^{2}+\alpha^{5}+\alpha^{4}\left(-1-\alpha^{3}\right) \\
& = & \alpha^{2}+\alpha^{5}-\alpha^{4}-\alpha^{7} \\
& = & -\left(\frac{1}{\alpha}+\alpha+\alpha^{4}+\alpha^{6}\right) .
\end{array}
$$

Portanto, $g_{1}\left(\mathcal{R}_{\alpha-1}\right) \cap g_{2}\left(\mathcal{R}_{\alpha-1}\right)=\left\{-\left(\alpha^{-1}+\alpha+\alpha^{4}+\alpha^{6}\right)\right\}=g_{1}(u)=g_{2}(u)$.

4. Suponha $\gamma \in g_{0}\left(\mathcal{R}_{\alpha-1}\right) \cap g_{2}\left(\mathcal{R}_{\alpha-1}\right)$.

Então existem $y, z \in \mathcal{R}_{\alpha-1}$ tais que $\gamma=g_{0}(y)=g_{2}(z)$ ou seja $\gamma=\alpha^{-1}+\alpha^{-3}+\alpha^{2} y=$ $\alpha^{2}+\alpha^{5}+\alpha^{4} z$. Desta forma,

$$
y=\alpha-1+\alpha^{3}+\alpha^{2} z=\alpha^{-3}+\alpha^{-1}+\alpha^{3}+\alpha^{2} z
$$

Por (4.9) podemos escrever os elementos de $\mathcal{R}_{\alpha-1}$ de duas maneiras, considerando a primeira: $z=$ $\alpha-1+\alpha^{4}+\alpha^{3} \widetilde{z}, \widetilde{z} \in \mathcal{R}$ substituindo em (4.12) e se $\alpha^{4}-2 \alpha^{3}+\alpha^{2}-\alpha=0$ então

$$
\begin{array}{rlc}
y & = & \alpha^{-1}+\alpha^{-3}+\alpha^{3}+\alpha^{2} z \\
& = & \alpha^{-1}+\alpha^{-3}+\alpha^{3}+\alpha^{2}\left(\alpha-1+\alpha^{4}+\alpha^{3} \widetilde{z}\right) \\
& = & \alpha-1+2 \alpha^{3}-\alpha^{2}+\alpha^{6}+\alpha^{5} \widetilde{z} \\
& = & -1+\left(\alpha+2 \alpha^{3}\right)-\alpha^{2}+\alpha^{6}+\alpha^{5} \widetilde{z} \\
& = & -1+\alpha^{4}+\alpha^{2}-\alpha^{2}+\alpha^{6}+\alpha^{5} \widetilde{z} \\
& = & -1+\alpha^{4}+\alpha^{6}+\alpha^{5} \widetilde{z} .
\end{array}
$$

Logo, $y=-1-\alpha^{3}=u \in \mathcal{R} \cap(\mathcal{R}+\alpha-1) \cap(\mathcal{R}-1)$.

Agora, considerando a segunda maneira $z=\alpha^{2}+\alpha^{3} \widehat{z}, \widehat{z} \in \mathcal{R}$ e novamente substituindo em (4.12) temos,

$y=\alpha-1+\alpha^{3}+\alpha^{2} z=\underbrace{\alpha^{-1}+\alpha^{-3}}_{\alpha-1}+\alpha^{3}+\alpha^{2}\left(\alpha^{2}+\alpha^{3} \widehat{z}\right)=-1+\alpha^{5}+\alpha^{5} \widehat{z}$.

Dos dois casos anteriores, concluímos que $y \in \mathcal{R} \cap(\mathcal{R}+\alpha-1) \cap(\mathcal{R}-1)$ e, portanto, pelo Lema (4.0.4), $y=-1-\alpha^{3}$. Assim, $\gamma=\alpha^{-1}+\alpha^{-3}+\alpha^{2} y=\alpha^{-1}+\alpha^{-3}+\alpha^{2}\left(-1-\alpha^{3}\right)=-\alpha^{-1}-\alpha-\alpha^{5}$. Agora tomando z, em (4.12) temos

$$
\alpha^{-1}+\alpha^{-3}+\alpha^{2} y=\alpha^{2}+\alpha^{5}+\alpha^{4} z \Rightarrow z=-\alpha^{-3}-\alpha^{-5}-\alpha+\alpha^{2} y
$$

Como $y \in \mathcal{R}_{\alpha-1}$ pode ser escrito de duas maneiras, considerando a primeira $y=\alpha-1+\alpha^{4}+$ $\alpha^{3} \widetilde{z}, \widetilde{z} \in \mathcal{R}$, temos substituindo em (4.13): $z=-\alpha^{-3}-\alpha^{-5}-\alpha+\alpha^{-2}\left(-\alpha^{-3}+\alpha^{-1}+\alpha^{4}+\alpha^{3} \widetilde{z}=\right.$ 
$-\alpha+\alpha^{2}+\alpha \widetilde{z}$.

Logo $z \in \mathcal{R} \cap(\mathcal{R}+\alpha-1) \cap(\mathcal{R}-\alpha)=\emptyset$.

Usando a segunda forma $y=\alpha^{2}+\alpha^{3} \widehat{z}, \widehat{z} \in \mathcal{R}$ na equação (4.13) temos, $z=-\alpha^{-3}-\alpha^{-5}-$ $\alpha+\alpha^{-2}\left(\alpha^{-3}+\alpha^{-1}+\alpha^{4}+\alpha^{3} \widetilde{z}\right)=-\alpha+\alpha^{2}+\alpha \widetilde{z} \Longrightarrow z \in \mathcal{R} \cap(\mathcal{R}+(\alpha-1)) \cap(\mathcal{R}-\alpha)$.

Considerando a segunda forma $y=\alpha^{2}+\alpha^{3} \widehat{z}, \widehat{z} \in \mathcal{R}$ temos:

$$
\begin{aligned}
z & = & -\alpha^{-3}-\alpha^{-5}-\alpha+\alpha^{-2}\left(\alpha^{2}+\alpha^{3} \widehat{z}\right) \\
& = & -\alpha^{-3}-\alpha^{-5}-\alpha+1+\alpha \widehat{z} \\
& = & -2 \alpha^{-3}-\alpha^{-5}-\alpha^{-1}+\alpha \widehat{z} \\
& = & -1+\alpha \widehat{z} .
\end{aligned}
$$

Logo, $z \in \mathcal{R} \cap(\mathcal{R}+\alpha-1) \cap(\mathcal{R}-1)$ e pelo Lema (4.0.4) temos que $z=-1-\alpha^{3}$.

Agora se $z=-1-\alpha^{3}$ substituindo em (4.13) temos

$$
\begin{array}{ccc}
\alpha^{-1}+\alpha^{-3}+\alpha^{2}\left(-1-\alpha^{3}\right) & = & \alpha^{2}+\alpha^{5}+\alpha^{4}\left(-1-\alpha^{3}\right) \\
\alpha-1-\alpha^{2}-\alpha^{5} & = & \alpha^{2}+\alpha^{5}-\alpha^{4}-\alpha^{7} \\
\alpha+\alpha^{4}+\alpha^{7} & = & 1+2 \alpha^{2}+2 \alpha^{5} \\
\alpha+\alpha^{4}+\alpha^{7} & = & 1+2 \alpha^{2}-\alpha^{3}+\alpha^{4}+\alpha^{6} \\
\alpha^{7} & = & 1+2 \alpha^{2}-\alpha-\alpha^{3}+\alpha^{6} \\
\alpha^{7} & = & \alpha^{6}, \text { o que não ocorre! }
\end{array}
$$

Portanto, $g_{0}\left(\mathcal{R}_{\alpha-1}\right) \cap g_{2}\left(\mathcal{R}_{\alpha-1}\right)=\emptyset$.

Lema 4.0.6 As seguintes relações são verificadas:

1. $\mathcal{R}_{1}=-\alpha+\alpha^{-1} \mathcal{R}_{\alpha-1}$;

2. $\mathcal{R}_{\alpha}=-\alpha^{2}+\mathcal{R}_{\alpha-1}$;

3. $\mathcal{R}_{1-\alpha}=-\alpha+1+\mathcal{R}_{\alpha-1}$;

4. $\mathcal{R}_{-\alpha}=-\alpha-\alpha^{2}+\mathcal{R}_{\alpha-1}$

5. $\mathcal{R}_{-1}=-1-\alpha+\alpha^{-1} \mathcal{R}_{\alpha-1}$

\section{Demonstração:}


1. Seja $z \in \mathcal{R}_{1}$, então $z=1+\sum_{i=2}^{\infty} \varepsilon_{i} \alpha^{i}=\sum_{i=2}^{\infty} \varepsilon_{i}^{\prime} \alpha^{i}$, onde $\left(\varepsilon_{i}\right)_{i \geq 2},\left(\varepsilon_{i}^{\prime}\right)_{i \geq 2} \in \mathcal{D}^{\infty}$. Vamos mostrar que $z \in-\alpha+\alpha^{-1} \mathcal{R}_{\alpha-1}$. Como

$$
\alpha-1=\alpha^{-3}+\alpha^{-1}
$$

então $(1,0)(0,0)\left(\varepsilon_{2}, \varepsilon_{2}^{\prime}\right),(0,0)\left(\varepsilon_{4}, \varepsilon_{4}\right) \ldots$ é um caminho no autômato duplo começando no estado inicial.

Analisando o autômato temos três possibilidades: $\left(\varepsilon_{2}, \varepsilon_{2}^{\prime}\right)(0,0)\left(\varepsilon_{4}, \varepsilon_{4}^{\prime}\right)=(1,0)(0,0)(0,0)$ ou $\left(\varepsilon_{2}, \varepsilon_{2}^{\prime}\right)(0,0)\left(\varepsilon_{4}, \varepsilon_{4}^{\prime}\right)=(1,0)(0,0)(1,1)$ ou $\left(\varepsilon_{2}, \varepsilon_{2}^{\prime}\right)(0,0)\left(\varepsilon_{4}, \varepsilon_{4}^{\prime}\right)=(1,0)(0,0)(0,1)$.

Caso 1: Se $\left(\varepsilon_{2}, \varepsilon_{2}^{\prime}\right)(0,0)\left(\varepsilon_{4}, \varepsilon_{4}^{\prime}\right)=(1,0)(0,0)(0,0)$ então temos $z=1+\alpha^{2}+\alpha^{5} w_{1}=\alpha^{5} w_{1}^{\prime}$ onde $w_{1}, w_{1}^{\prime} \in \mathcal{R}$. Assim $\alpha^{2}+\alpha z=\alpha^{2}+\alpha\left(1+\alpha^{2}+\alpha^{5} w_{1}\right)=\alpha^{2}+\alpha^{5} w_{1}^{\prime}$. Por outro lado $\alpha^{2}+\alpha z=(\alpha-1)+\alpha^{4}+\alpha^{6} w_{1}$.

Portanto, $\alpha^{2}+\alpha z \in(\mathcal{R}+\alpha-1) \cap \mathcal{R}=\mathcal{R}_{\alpha-1}$, logo $z \in-\alpha+\alpha^{-1} \mathcal{R}_{\alpha-1}$.

Caso 2: Se $\left(\varepsilon_{2}, \varepsilon_{2}^{\prime}\right)(0,0)\left(\varepsilon_{4}, \varepsilon_{4}^{\prime}\right)=(1,0)(0,0)(1,1)$ entâo temos $z=1+\alpha^{2}+\alpha^{4}+\alpha^{5} w_{2}=$ $\alpha^{4}+\alpha^{5} w_{2}^{\prime}$ onde $w_{2}, w_{2}^{\prime} \in \mathcal{R}$. Logo $\alpha^{2}+\alpha z=\alpha^{2}+\alpha\left(1+\alpha^{2}+\alpha^{4}+\alpha^{5} w_{2}\right)=\alpha^{2}+\alpha\left(\alpha^{4}+\alpha^{5} w_{2}^{\prime}\right)$. Reciprocamente, $\alpha^{2}+\alpha z=(\alpha-1)+\left(1+\alpha^{2}+\alpha^{3}\right)+\alpha^{5}+\alpha^{6} w_{2}=(\alpha-1)+\alpha^{4}+\alpha^{5}+\alpha^{6} w_{2}$. Assim, $\alpha^{2}+\alpha z \in(\mathcal{R}+\alpha-1) \cap \mathcal{R}=\mathcal{R}_{\alpha-1}$, então $z \in-\alpha+\alpha^{-1} \mathcal{R}_{\alpha-1}$.

Caso 3: Se $\left(\varepsilon_{2}, \varepsilon_{2}^{\prime}\right)(0,0)\left(\varepsilon_{4}, \varepsilon_{4}^{\prime}\right)=(1,0)(0,0)(0,1)$ então temos $z=1+\alpha^{2}+\alpha^{5} w_{3}=\alpha^{4}+\alpha^{5} w_{3}^{\prime}$ onde $w_{3}, w_{3}^{\prime} \in \mathcal{R}$. Logo $\alpha^{2}+\alpha z=\alpha^{2}+\alpha\left(1+\alpha^{2}+\alpha^{5} w_{3}\right)=\alpha^{2}+\alpha\left(\alpha^{4}+\alpha^{5} w_{3}^{\prime}\right)$.

Agora, temos que $\alpha^{2}+\alpha z=\alpha^{2}+\alpha+\alpha^{3}+\alpha^{6} w_{3}=(\alpha-1)+\alpha^{4}+\alpha^{6} w_{3}$.

Portanto, $\alpha^{2}+\alpha z \in(\mathcal{R}+\alpha-1) \cap \mathcal{R}=\mathcal{R}_{\alpha-1}$, então $z \in-\alpha+\alpha^{-1} \mathcal{R}_{\alpha-1}$.

Dos três casos acima, segue que $\alpha^{2}+\alpha z \in \mathcal{R}_{\alpha-1}$.

Reciprocamente, se $x \in \mathcal{R}_{\alpha-1}=g_{0}\left(\mathcal{R}_{\alpha-1}\right) \cup g_{1}\left(\mathcal{R}_{\alpha-1}\right) \cup g_{2}\left(\mathcal{R}_{\alpha-1}\right)$ então se $x \in g_{0}\left(\mathcal{R}_{\alpha-1}\right)$ usando a relação (4.9) podemos escrevê-lo de duas maneiras: considerando a primeira $x=$ $g_{0}\left(\alpha^{-3}+\alpha^{-1}+\alpha^{4}+\alpha^{3} z_{1}\right)=\alpha^{2}+\alpha^{6}+\alpha^{5} z_{1}$, onde $z_{1} \in \mathcal{R}_{\alpha-1}$ temos que $-\alpha+\alpha^{-1} x=$ $-\alpha+\alpha^{-1}\left(\alpha^{2}+\alpha^{6}+\alpha^{5} z_{1}\right)=-\alpha+\alpha+\alpha^{5}+\alpha^{4} z_{1}=\alpha^{5}+\alpha^{4} z_{1} \in \mathcal{R}$.

Agora, considerando a segunda $x=g_{0}\left(\alpha^{2}+\alpha^{3} z_{2}\right)=\alpha-1+\alpha^{4}+\alpha^{5} z_{2}, z_{2} \in \mathcal{R}$ logo $-\alpha+\alpha^{-1} x=$ $-\alpha+1-\alpha^{-1}+\alpha^{3}+\alpha^{4} z_{2}=-\alpha+1-\alpha^{-1}+\alpha^{2}+\alpha+\alpha^{-1}+\alpha^{4} z_{2}=1+\alpha^{2}+\alpha^{4} z_{2} \in \mathcal{R}+1$. Portanto, $-\alpha-\alpha^{-1} x \in \mathcal{R}_{1}$.

Se $x \in g_{1}\left(\mathcal{R}_{\alpha-1}\right)$ usando o mesmo raciocínio anterior temos $x=g_{1}\left(\alpha^{-3}+\alpha^{-1}+\alpha^{4}+\alpha^{3} z_{3}\right)=$ $\alpha^{2}+\alpha^{5}+\alpha^{6}+\alpha^{6} z_{3}$, onde $z_{3} \in \mathcal{R}$ temos que $-\alpha+\alpha^{-1} x=\alpha^{4}+\alpha^{5}+\alpha^{6} z_{3} \in \mathcal{R}$. Tomando 
agora $x=g_{1}\left(\alpha^{2}+\alpha^{3} z_{4}\right)=\alpha-1+\alpha^{4}+\alpha^{6} z_{4}, z_{4} \in \mathcal{R}$ então temos $-\alpha+\alpha^{-1} x=-\alpha-\alpha^{-1}+$ $1+\alpha^{2}+\alpha+\alpha^{-1}+\alpha^{5} z_{4}=1+\alpha^{2}+\alpha^{5} z_{4} \in \mathcal{R}+1$.

Finalmente, se $x \in g_{2}\left(\mathcal{R}_{\alpha-1}\right)$ considerando $x=g_{2}\left(\alpha^{-3}+\alpha^{-1}+\alpha^{4}+\alpha^{3} z_{5}\right)=\alpha+\alpha^{2}+\alpha^{3}+\alpha^{5}+$ $\alpha^{8}+\alpha^{7} z_{5}$, onde $z_{5} \in \mathcal{R}_{\alpha-1}$ então temos que $-\alpha+\alpha^{-1} x=1+\alpha^{2}+\alpha^{4}+\alpha^{7}+\alpha^{6} z_{5} \in \mathcal{R}+1$. Tomando agora $x=g_{2}\left(\alpha^{2}+\alpha^{3} z_{6}\right)=\alpha^{2}+\alpha^{5}+\alpha^{6}+\alpha^{7} z_{6}, z_{6} \in \mathcal{R}$ temos $-\alpha+\alpha^{-1} x=$ $\alpha^{4}+\alpha^{5}+\alpha^{6} z_{6} \in \mathcal{R}$.

Portanto, $-\alpha+\alpha^{-1} \mathcal{R}_{\alpha-1} \subset \mathcal{R}_{1}$.

2. Seja $z \in \mathcal{R}_{\alpha}$, então $z=\alpha+\sum_{i=2}^{\infty} \varepsilon_{i} \alpha^{i}=\sum_{i=2}^{\infty} \varepsilon_{i}^{\prime} \alpha^{i}$, onde $\left(\varepsilon_{i}\right)_{i \geq 2}, \quad\left(\varepsilon_{i}^{\prime}\right)_{i \geq 2} \in \mathcal{D}^{\infty}$ logo $(1,0)\left(\varepsilon_{2}, \varepsilon_{2}^{\prime}\right)\left(\varepsilon_{3}, \varepsilon_{3}^{\prime}\right)$.. é um caminho no autômato duplo começando no estado inicial. Analisando o autômato temos as seguintes possibilidades: $\left(\varepsilon_{2}, \varepsilon_{2}^{\prime}\right),\left(\varepsilon_{3}, \varepsilon_{3}^{\prime}\right)\left(\varepsilon_{4}, \varepsilon_{4}^{\prime}\right)=(0,0)(1,0)(0,0)$ ou $\left(\varepsilon_{2}, \varepsilon_{2}^{\prime}\right)\left(\varepsilon_{3}, \varepsilon_{3}^{\prime}\right)=(0,1)(1,0)$.

Caso 1: Se $\left(\varepsilon_{2}, \varepsilon_{2}^{\prime}\right)\left(\varepsilon_{3}, \varepsilon_{3}^{\prime}\right)\left(\varepsilon_{4}, \varepsilon_{4}^{\prime}\right)=(0,0)(1,0)(0,0)$ então temos $z=\alpha+\alpha^{3}+\alpha^{5} t_{1}=\alpha^{5} t_{1}^{\prime}$ onde $t_{1}, t_{1}^{\prime} \in \mathcal{R} . A \operatorname{ssim} z+\alpha^{2}=\alpha+\alpha^{2}+\alpha^{3}+\alpha^{5} t_{1}=\underbrace{\alpha^{2}+\alpha^{5} t_{1}^{\prime}}_{\in \mathcal{R}}$. Logo $z+\alpha^{2}=\alpha+\alpha^{2}+\alpha^{3}+\alpha^{5} t_{1}=$ $(\alpha-1)+\alpha^{4}+\alpha^{5} t_{1} \in \mathcal{R}+\alpha-1$.

Portanto, $z+\alpha^{2} \in \mathcal{R}+(\alpha-1) \cap \mathcal{R}=\mathcal{R}_{\alpha-1}$ e assim $z \in-\alpha^{2}+\mathcal{R}_{\alpha-1}$.

Por outro lado, se $x \in \mathcal{R}_{\alpha-1}=g_{0}\left(\mathcal{R}_{\alpha-1}\right) \cup g_{1}\left(\mathcal{R}_{\alpha-1}\right) \cup g_{2}\left(\mathcal{R}_{\alpha-1}\right)$ então se $x \in g_{0}\left(\mathcal{R}_{\alpha-1}\right)$ usando a relação (4.9) podemos escrevê-lo de duas maneiras. Considerando a primeira $x=$ $g_{0}\left(\alpha^{-3}+\alpha^{-1}+\alpha^{4}+\alpha^{3} t_{2}\right)=\alpha^{2}+\alpha^{6}+\alpha^{5} t_{2}$, onde $t_{2} \in \mathcal{R}_{\alpha-1}$ então $-\alpha^{2}+x=\alpha^{6}+\alpha^{5} t_{2} \in \mathcal{R}$. Agora, se $x=g_{0}\left(\alpha^{2}+\alpha^{3} t_{2}^{\prime}\right)=\alpha-1+\alpha^{4}+\alpha^{5} t_{2}^{\prime}, t_{2}^{\prime} \in \mathcal{R}_{\alpha-1}$ ent $\tilde{a} o-\alpha^{2}+x=\alpha+\alpha^{3}+\alpha^{5} t_{2}^{\prime} \in$ $\mathcal{R}+\alpha$.

Se $x=g_{1}\left(\alpha^{-3}+\alpha^{-1}+\alpha^{4}+\alpha^{3} t_{2}\right)=\alpha^{2}+\alpha^{5}+\alpha^{6} t_{2}$, onde $t_{2} \in \mathcal{R}_{\alpha-1}$ então temos que $-\alpha^{2}+x=\alpha^{5}+\alpha^{6} t_{2} \in \mathcal{R}$.

E se $x=g_{1}\left(\alpha^{2}+\alpha^{3} t_{2}^{\prime}\right)=\alpha-1+\alpha^{4}+\alpha^{6} t_{2}^{\prime}, t_{2}^{\prime} \in \mathcal{R}_{\alpha-1}$ então $-\alpha^{2}+x=\alpha+\alpha^{3}+\alpha^{6} t_{2}^{\prime} \in \mathcal{R}+\alpha$. E finalmente, se $x=g_{2}\left(\alpha^{-3}+\alpha^{-1}+\alpha^{4}+\alpha^{3} t_{2}\right)=\alpha-1+\alpha^{4}+\alpha^{5}+\alpha^{8}+\alpha^{7} t_{2}$, onde $t_{2} \in \mathcal{R}_{\alpha-1}$ ent $\tilde{a} o-\alpha^{2}+x=\alpha+\alpha^{3}+\alpha^{5}+\alpha^{8}+\alpha^{7} t_{2} \in \mathcal{R}+\alpha$. E se $x=g_{2}\left(\alpha^{2}+\alpha^{3} t_{2}^{\prime}\right)=$ $\alpha^{2}+\alpha^{5}+\alpha^{6}+\alpha^{7} t_{2}^{\prime}, t_{2}^{\prime} \in \mathcal{R}_{\alpha-1}$ então $-\alpha^{2}+x=\alpha^{5}+\alpha^{6}+\alpha^{7} t_{2}^{\prime} \in \mathcal{R}$.

Portanto, das análises anteriores temos que $-\alpha^{2}+\mathcal{R}_{\alpha-1} \subset \mathcal{R}_{\alpha}$ e assim $\mathcal{R}_{\alpha}=-\alpha^{2}+\mathcal{R}_{\alpha-1}$. Caso 2: Se $\left(\varepsilon_{2}, \varepsilon_{2}^{\prime}\right)\left(\varepsilon_{3}, \varepsilon_{3}^{\prime}\right)=(0,1)(1,0)$ então temos $z=\alpha+\alpha^{3}+\alpha^{4} w_{3}=\alpha^{2}+\alpha^{4} w_{3}^{\prime}$ onde $w_{3}, w_{3}^{\prime} \in \mathcal{R}$. Assim $z+\alpha^{2}=\alpha+\alpha^{2}+\alpha^{3}+\alpha^{4} w_{3}=2 \alpha^{2}+\alpha^{4} w_{3}^{\prime}$. Logo $z+\alpha^{2}=$ $\alpha+\alpha^{2}+\alpha^{3}+\alpha^{5} t_{1}=(\alpha-1)+\alpha^{4}+\alpha^{5} t_{1} \in \mathcal{R}+\alpha-1$. 


\subsection{Parametrização de $\mathcal{R}_{\alpha-1}$}

Seja z um elemento de $\mathcal{R}_{\alpha-1}$. Pelo Lema 4.0.5, existem $z_{1} \in \mathcal{R}_{\alpha-1}$ e $a_{1}$ elemento de $A=$ $\{0,1,2\}$ tais que $z=g_{a_{1}}\left(z_{1}\right)$. Continuando assim construímos uma sequência $\left(a_{n}\right)_{n \geq 1}$ em $\{0,1,2\}^{\mathbb{N}}$ e uma sequência $\left(z_{n}\right)_{n \geq 1}$ em $\mathcal{R}_{\alpha-1}$, tais que para todo $n$ :

$$
z=g_{a_{1}} \circ g_{a_{2}} \circ \ldots \circ g_{a_{n}}\left(z_{n}\right)
$$

Como as funções $g_{i}$ são contrações, então para todo $x \in \mathcal{R}_{\alpha-1}$, a sequência $g_{a_{1}} \circ g_{a_{2}} \circ \ldots \circ g_{a_{n}}(x)$ converge para $z$ quando $n$ tende a infinito.

Seja

$$
\begin{aligned}
& \psi: A^{\mathbb{N}} \longrightarrow A^{\mathbb{N}} \\
& a_{1} a_{2} \ldots \longmapsto b_{1} b_{2} \ldots
\end{aligned}
$$

definida como segue:

Temos $b_{1}=a_{1}$.

Seja $k \geq 2$, definimos $b_{k}$ da seguinte maneira:

$$
\text { Se } a_{k}=1 \text { então } b_{k}=1 \text {; }
$$

Se $a_{k} \neq 1$ e $\quad a_{k-1}=2 \quad$ então $\quad b_{k}=a_{k} ;$

$$
\begin{aligned}
\text { Se } a_{k} \neq 1 \text { e } \quad a_{k-1}=0 \quad \text { então } \quad & a) b_{k}=2 \text { se } a_{k}=0 \\
b) b_{k}=0 & \text { se } a_{k}=2 .
\end{aligned}
$$

Se $a_{k} \neq 1$ e $a_{k-1}=1$, seja $r=\min \left\{1 \leq i \leq k-1, a_{i}=a_{i+1}=\ldots=a_{k-1}=1\right\}$ :

- $\left(\right.$ Se $r>1$ e $\left.a_{r-1}=2\right)$ ou $r=1$ então

$$
\left\{\begin{array}{c}
a) b_{k}=a_{k}, \quad \text { se \# }(k-r) \quad \text { é par } \\
b) b_{k}=2-a_{k}, \quad \text { se \# }(k-r) \quad \text { é ímpar }
\end{array}\right.
$$

onde \#(k-r) é o número de algarismos 1 após um algarismo 0 ou 2.

- (Se $r>1$ e $\left.a_{r-1}=0\right)$ então

$$
\left\{\begin{array}{c}
a) b_{k}=a_{k}, \quad \text { se \#(k-r) é ímpar } \\
b) b_{k}=2-a_{k}, \quad \text { se \# }(k-r) \quad \text { é par }
\end{array}\right.
$$


Fixemos $x_{0} \in \mathcal{R}_{\alpha-1}$ e definimos $f:[0,1] \longrightarrow \mathcal{R}_{\alpha-1}$ a correspondência definida da seguinte maneira: se $t=\sum_{i=1}^{\infty} a_{i} 3^{-i}$ onde $\left(a_{i}\right)_{i \geq 0} \in A^{\mathbb{N}}$, então $f(t)=\lim _{n \longrightarrow \infty} g_{b_{1}} \circ \ldots \circ g_{b_{n}}\left(x_{0}\right)$ onde $b_{1} b_{2} \ldots=$ $\psi\left(a_{1} a_{2} \ldots\right)$.

Teorema 4.1.1 : A correspondência $f:[0,1] \longrightarrow \mathcal{R}_{\alpha-1}$ é uma função contínua, bijetora e satisfaz $f(0)=u$ e $f(1)=w$.

Se $t$ e $t^{\prime}$ pertencem a $[0,1]$ então $t=\sum_{i=1}^{\infty} a_{i} 3^{-i}$ e $t^{\prime}=\sum_{i=1}^{\infty} c_{i} 3^{-i}$ onde $a_{i}$ e $c_{i}$ são elementos de $\{0,1,2\}$ tais que $a_{i}=c_{i}$ para $i<k$ e $a_{k}<c_{k}$ onde $k \in \mathbb{N}$. Supomos que $f(t)=\lim _{n \longrightarrow \infty} g_{c_{1}} \circ \ldots \circ g_{c_{n}}\left(x_{0}\right)$ e $f\left(t^{\prime}\right)=\lim _{n \longrightarrow \infty} g_{d_{1}} \circ \ldots \circ g_{d_{n}}\left(x_{0}\right)$ onde $c_{1} c_{2} \ldots=\psi\left(a_{1} a_{2} \ldots\right)$ e $d_{1} d_{2} \ldots=\psi\left(b_{1} b_{2} \ldots\right)$.

Para demonstrar esta proposição serão necessários alguns resultados.

Lema 4.1.2 : Sejam $t$ e $t^{\prime}$ dois elementos de $[0,1]$ tais que $t=\sum_{i=1}^{\infty} a_{i} 3^{-i}$ e $t^{\prime}=\sum_{i=1}^{\infty} c_{i} 3^{-i}$ onde $a_{i}$ e $c_{i}$ são elementos de $\{0,1,2\}$ tais que $a_{i}=c_{i}$ para $i<k$ e $a_{k}<c_{k}$ onde $k \in \mathbb{N}^{\star}$.

1. Se $\left|t-t^{\prime}\right|<3^{-N}$ onde $N>k$, então $c_{k}=a_{k}+1, c_{i}=0$ e $a_{i}=2$ para todo $i$ que satisfaz $k+1 \leq i \leq N$.

2. Se $t=t^{\prime}$ então $c_{k}=a_{k}+1, c_{i}=0$ e $a_{i}=2$ para $i \geq k+1$.

\section{Demonstração:}

1 .

$$
\begin{array}{ccc}
3^{-N}> & \sum_{i=1}^{\infty}\left(c_{i}-a_{i}\right) 3^{-i}=\left(c_{k}-a_{k}\right) 3^{-K}+\sum_{i=k+1}^{\infty}\left(c_{i}-a_{i}\right) 3^{-i} \\
& = & \left(c_{k}-a_{k}-1\right) 3^{-k}+\sum_{i=k+1}^{\infty}\left(2+c_{i}-a_{i}\right) 3^{-i}
\end{array}
$$

2. A segunda relação segue imediatamente da primeira.

Vamos usar o seguinte lema para provar a proposição:

Lema 4.1.3 Sejam $f, g_{0}, g_{1}$ e $g_{2}$ definidas anteriormente. As seguintes propriedades são verificadas:

1)

$$
\lim _{n \longrightarrow \infty} g_{2}^{n}(z)=w=\alpha^{2}-\alpha^{3}
$$

2)

$$
\lim _{n \longrightarrow \infty} g_{1} \circ g_{2}^{n}(z)=\lim _{n \longrightarrow \infty} g_{0}^{2} \circ g_{2}^{n}(z)=g_{0}(u)=-\alpha^{-1}-\alpha-\alpha^{5}
$$


3)

$$
\lim _{n \longrightarrow \infty} g_{1} \circ g_{0} \circ g_{2}^{n}(z)=\lim _{n \longrightarrow \infty} g_{2} \circ g_{0} \circ g_{2}^{n}(z)=g_{1}(u)=-\alpha^{-1}-\alpha-\alpha^{4}-\alpha^{6} .
$$

\section{Demonstração:}

1) $\operatorname{Como~}_{2}(z)=\alpha^{2}+\alpha^{5}+\alpha^{4} z, z \in \mathbb{C}$, então $g_{2}^{2}(z)=\alpha^{2}+\alpha^{5}+\alpha^{6}+\alpha^{9}+\alpha^{8} z$. Podemos provar por indução que $g_{2}^{n}(z)=\sum_{i=0}^{n-1}\left(\alpha^{4 i+2}+\alpha^{4 i+5}\right)+\alpha^{4 n} z$. Assim, como $|\alpha|<1$ temos que $\lim _{n \longrightarrow \infty} g_{2}^{n}(z)=\lim _{n \longrightarrow \infty}\left(\sum_{i=0}^{\infty} \alpha^{4 i+2}+\alpha^{4 i+5}\right)+\lim _{n \longrightarrow \infty}\left(\alpha^{4 n} z\right)=\frac{\left(\alpha^{2}+\alpha^{5}\right)}{1-\alpha^{4}}=\frac{\left(\alpha^{6}-\alpha^{4}\right)}{-\alpha^{2}-\alpha^{3}}=$ $\alpha^{2}-\alpha^{3}=w$.

2) Como $g_{1}(z)=-1-\alpha^{3}+\alpha^{3} z$ e $g_{0}^{2}(z)=\alpha^{2}+\alpha^{4} z$ então $\lim _{n \longrightarrow \infty} g_{1}\left(g_{2}^{n}(z)\right)=g_{1}\left(\alpha^{2}-\alpha^{3}\right)=$ $-1-\alpha^{3}+\alpha^{3}\left(\alpha^{2}-\alpha^{3}\right)=-\left(\alpha^{5}+\alpha+\alpha^{-1}\right)=g_{0}(u)$

Assim, $\lim _{n \longrightarrow \infty} g_{1} \circ g_{2}^{n}(z)=\lim _{n \longrightarrow \infty} g_{0}^{2} \circ g_{2}^{n}(z)$

3) Como $_{1} \circ g_{0}(z)=g_{1}\left(\alpha^{-3}+\alpha^{-1}+\alpha^{2} z\right)=-\alpha^{-1}-\alpha+\alpha^{5} z$ e $g_{2} \circ g_{0}(z)=g_{2}\left(\alpha^{-3}+\alpha^{-1}+\alpha^{2} z\right)=$ $\alpha^{2}+\alpha^{5}+\alpha+\alpha^{3}+\alpha^{6} z$ então $\lim _{n \longrightarrow \infty} g_{1} \circ g_{0}\left(g_{2}^{n}(z)\right)=g_{1} \circ g_{0}\left(\alpha^{2}-\alpha^{3}\right)=-\left(\alpha^{-1}+\alpha+\alpha^{4}+\alpha^{6}\right)=$ $g_{0}(u)$ e $\lim _{n \longrightarrow \infty} g_{2} \circ g_{0} \circ g_{2}^{n}(z)=g_{2} \circ g_{0}\left(\alpha^{2}-\alpha^{3}\right)=\alpha^{2}+\alpha^{5}+\alpha+\alpha^{3}+\alpha^{6}\left(\alpha^{2}-\alpha^{3}\right)=$ $-\left(\alpha^{-1}+\alpha+\alpha^{4}+\alpha^{6}\right)$.

Portanto, $\lim _{n \longrightarrow \infty} g_{1} \circ g_{0} \circ g_{2}^{n}(z)=\lim _{n \longrightarrow \infty} g_{2} \circ g_{0} \circ g_{2}^{n}(z)$.

\section{Prova do Teorema:}

fé bem definida: Sejam $t, t^{\prime} \in[0,1]$ tais que $t=\sum_{i=1}^{\infty} a_{i} 3^{-i}$ e $t^{\prime}=\sum_{i=1}^{\infty} a_{i}^{\prime} 3^{-i}$. Suponhamos que $t=t^{\prime}$ e que $\left(a_{i}\right)_{i \geq 2} \leq_{\text {lex }}\left(a_{i}^{\prime}\right)_{i \leq 2}$. Por Teorema temos que $a_{i}=a_{i}^{\prime}$ para $i<k$ e que existe um $k$ tal que $a_{k}^{\prime}=a_{k}+1, a_{i}^{\prime}=0, i \geq k+1, a_{i}^{\prime}=2, i \geq k+1$.

Sejam $\left(b_{i}\right)$ e $\left(c_{i}\right)$ as imagens de $\left(a_{i}\right)$ e $\left(a_{i}^{\prime}\right)$ por $\psi$, respctivamente.

Sem perda de generalidade vamos supor $t=\left(a_{1} a_{2} \ldots a_{k-1} 1 \overline{2}\right)$ e $t^{\prime}=\left(a_{1} a_{2} \ldots a_{k-1} 2 \overline{0}\right), k \geq 2$, onde $\overline{2}=222 \ldots$ e $\overline{0}=000 \ldots$

Caso 1: Se $a_{k-1}=0$ então $t=\left(a_{1} a_{2} \ldots a_{k-2} 01 \overline{2}\right)$ e $t^{\prime}=\left(a_{1} a_{2} \ldots a_{k-2} 02 \overline{0}\right)$. Logo $\psi(t)=$ $\left(b_{1} b_{2} \ldots b_{k-2} b_{k-1} b_{k} \ldots\right)$ e $\psi\left(t^{\prime}\right)=\left(b_{1} b_{2} \ldots b_{k-2} b_{k-1} c_{k} c_{k+1} \ldots\right)$. Vamos determinar $b_{k} b_{k+1} \ldots e$ $c_{k} c_{k+1} \ldots$

Se $a_{k}=1$ então pela relação (4.15) temos que $b_{k}=1$. Como $a_{k+1}=2$ e $a_{k}=1$, da relação (4.19)(a) segue que $b_{k+1}=2=a_{k+1}$. Como $a_{j}=2$, para todo $j \geq k+2$, então por (4.16) temos que $b_{j}=2$, para todo $j \geq k+2$.

Agora vamos determinar $c_{k} c_{k+1} \ldots$ Como $a_{k-1}=0$ e $a_{k}^{\prime}=2$ então pela relação (4.17)(b) temos que $c_{k}=0$. Como $a_{k}^{\prime}=2$ e $a_{k+1}^{\prime}=0$ então por (4.16) $c_{k+1}=a_{k+1}^{\prime}=0$. Como $a_{l}^{\prime}=0$ para todo $l \geq k+2$ entâo por (4.17)(a) $c_{l}=2$, para todo $l \geq k+2$. 
Assim, $\psi(t)=\left(b_{1} b_{2} \ldots b_{k-1} 1 \overline{2}\right)$ e $\psi\left(t^{\prime}\right)=\left(b_{1} b_{2} \ldots b_{k-1} 00 \overline{2}\right)$.

Logo, $f(t)=\lim _{n \longrightarrow \infty} g_{b_{1}} \circ g_{b_{2}} \circ \ldots \circ g_{b_{k-1}} \circ g_{1} \circ g_{2}^{n}\left(x_{0}\right)=g_{b_{1}} \circ g_{b_{2}} \circ \ldots \circ g_{b_{k-1}}\left(\lim _{n \longrightarrow \infty} g_{1} \circ g_{2}^{n}\left(x_{0}\right)\right)$.

Portanto, pela equação (4.21) do Lema (4.1.3) temos que $f(t)=f\left(t^{\prime}\right)$.

Caso 2: Se $a_{k-1}=2$ então $t=\left(a_{1} a_{2} \ldots a_{k-2} 21 \overline{2}\right)$ e $t^{\prime}=\left(a_{1} a_{2} \ldots a_{k-2} 22 \overline{0}\right)$. Então $\psi(t)=$ $\left(b_{1} b_{2} \ldots b_{k-2} b_{k-1} \ldots\right)$ e $\psi\left(t^{\prime}\right)=\left(b_{1} b_{2} \ldots b_{k-1} c_{k} c_{k+1} \ldots\right)$.

Como $a_{k}=1$ então pela relação (4.15) $b_{k}=1$. Como $a_{k+2}=2$ e $a_{k+1}=1$ então por (4.18)(b) $b_{k+1}=2-a_{k+1}=2-2=0$. Como $a_{k+2}=2$ então por (4.16) $b_{k+2}=2$.

Assim $\psi(t)=\left(b_{1} b_{2} \ldots b_{k-1} 10 \overline{2}\right)$.

Vamos determinar $c_{k} c_{k+1} \ldots$ se $a_{k-1}=2$ então por (4.16) $c_{k}=a_{k}^{\prime}=2$. Se $a_{k}^{\prime}=2$ então por (4.16) $c_{k+1}=a_{k+1}^{\prime}=0$. Se $a_{i}^{\prime}=0$ para todo $i \geq k+2$ então pela relação (4.17)(a) $c_{i}=2$ para todo $i \geq k+2$. Logo $\psi\left(t^{\prime}\right)=\left(b_{1} b_{2} \ldots b_{k-1} 20 \overline{2}\right)$.

Logo,

$f(t)=\lim _{n \longrightarrow \infty} g_{b_{1}} \circ g_{b_{2}} \circ \ldots \circ g_{b_{k-1}} \circ g_{1} \circ g_{0} \circ g_{2}^{n}\left(x_{0}\right) \quad$ e $f\left(t^{\prime}\right)=\lim _{n \longrightarrow \infty} g_{b_{1}} \circ g_{b_{2}} \circ \ldots \circ g_{b_{k-1}} \circ g_{2} \circ g_{0} \circ g_{2}^{n}\left(x_{0}\right)$.

Temos que $f(t)=\lim _{n \longrightarrow \infty} g_{b_{1}} \circ g_{b_{2}} \circ \ldots \circ g_{b_{k-1}} \circ g_{1} \circ g_{0} \circ g_{2}^{n}\left(x_{0}\right)=g_{b_{1}} \circ g_{b_{2}} \circ \ldots \circ g_{b_{k-1}}\left(\lim _{n \longrightarrow \infty} g_{1} \circ\right.$ $\left.g_{0} \circ g_{2}^{n}\left(x_{0}\right)\right)$. Pela equação (4.22) do Lema (4.1.3) temos $f(t)=g_{b_{1}} \circ g_{b_{2}} \circ \ldots \circ g_{b_{k-1}}\left(-\left(\alpha^{-1}+\alpha+\alpha^{4}+\right.\right.$ $\left.\left.\alpha^{6}\right)\right)$ e $f\left(t^{\prime}\right)=g_{b_{1}} \circ g_{b_{2}} \circ \ldots \circ g_{b_{k-1}}\left(\lim _{n \longrightarrow \infty} g_{2} \circ g_{0} \circ g_{2}^{n}\left(x_{0}\right)\right)=g_{b_{1}} \circ g_{b_{2}} \circ \ldots \circ g_{b_{k}-1}\left(-\left(\alpha^{-1}+\alpha+\alpha^{4}+\alpha^{6}\right)\right)$. Portanto, $f(t)=f\left(t^{\prime}\right)$.

Caso 3: Se $a_{k-1}=1$ então vamos subdividir este item em 3 casos:

1. Set $=\left(a_{1} a_{2} \ldots a_{r-2} 0 a_{r} \ldots a_{k-1} 1 \overline{2}\right)$ onde $a_{r}=\ldots=a_{k-1}=1$ e $t^{\prime}=\left(a_{1} a_{2} \ldots a_{r-2} 0 a_{r} \ldots a_{k-1} 2 \overline{0}\right)$ onde $a_{r}=\ldots=a_{k-1}=1 e \#(k-r)$ é par então pela relação (4.15) se $a_{k}=1$ então $b_{k}=1$ e por (4.19)(a) $b_{k+1}=2=a_{k+1}$.

Vamos determinar $c_{k} c_{k+1} \ldots$ como $a_{k}^{\prime}=2 e \#(k-r)$ é par então pela equação (4.19)(b) $c_{k}=2-a_{k}^{\prime}=0$. Se $a_{k+1}^{\prime}=0$ e $a_{k}^{\prime}=2$ então por (4.16) $c_{k+1}=0$. Finalmente, se $a_{i}^{\prime}=0$, para todo $i \geq k+1$ então por (4.17)(a) $c_{i}=2$ para todo $i \geq k+1$. Logo, $\psi(t)=\left(b_{1} b_{2} b_{k-1} 1 \overline{2}\right)$ e $\psi\left(t^{\prime}\right)=\left(b_{1} b_{2} \ldots b_{k-1} 00 \overline{2}\right)$.

Assim,

$f(t)=\lim _{n \longrightarrow \infty} g_{b_{1}} \circ g_{b_{2}} \circ \ldots \circ g_{b_{k-1}} \circ g_{1} \circ g_{2}^{n}\left(x_{0}\right) \quad$ e $f\left(t^{\prime}\right)=\lim _{n \longrightarrow \infty} g_{b_{1}} \circ g_{b_{2}} \circ \ldots \circ g_{b_{k-1}} \circ g_{0}^{2} \circ g_{2}^{n}\left(x_{0}\right)$.

Este caso é igual ao Caso 1.

Se $a_{k}=1$ então pela equação (4.15) temos $b_{k}=1$. Se \# $(k-r)$ é impar então por (4.19) 
$b_{k+1}=2-a_{k+1}=2-2=0$. Como $a_{i}=2$, para todo $i \geq k+1$ então por (4.16) $b_{i}=2$ para todo $i \geq k+2$.

Vamos determinar $c_{k} c_{k+1} \ldots$ se \# $(k-r)$ é ímpar então $c_{k}=a_{k}^{\prime}=2$. Como $a_{k}^{\prime}=2$ então por (4.16) $c_{k+1}=a_{k+1}^{\prime}=0$. Agora, como $a_{k+1}^{\prime}=0$ e $a_{k+2}^{\prime}=0$ então pela equação (4.17)(b) $c_{k+2}=2$. Logo, $\psi(t)=\left(b_{1} b_{2} b_{k-1} 10 \overline{2}\right)$ e $\psi\left(t^{\prime}\right)=\left(b_{1} b_{2} \ldots b_{k-1} 20 \overline{2}\right)$.

Assim,

$f(t)=\lim _{n \longrightarrow \infty} g_{b_{1}} \circ g_{b_{2}} \circ \ldots \circ g_{b_{k-1}} \circ g_{1} \circ g_{0} \circ g_{2}^{n}\left(x_{0}\right) \quad$ e $f\left(t^{\prime}\right)=\lim _{n \longrightarrow \infty} g_{b_{1}} \circ g_{b_{2}} \circ \ldots \circ g_{b_{k-1}} \circ g_{2} \circ g_{0} \circ g_{2}^{n}\left(x_{0}\right)$.

Este caso é igual ao Caso 2.

2. $S e t=\left(a_{1} a_{2} \ldots a_{r-2} 2 a_{r} \ldots a_{k-1} 1 \overline{2}\right)$ onde $a_{r}=\ldots=a_{k-1}=1$, e $t^{\prime}=\left(a_{1} a_{2} \ldots a_{r-2} 2 a_{r} \ldots a_{k-1} 2 \overline{0}\right)$ onde $a_{r}=\ldots=a_{k-1}=1$.

Se $a_{k}=1$ então pela equação (4.15) $b_{k}=1$. Se \# $(k-r)$ é par então por (4.18)(b) $b_{k+1}=$ $2-a_{k+1}=2-2=0$ e como $a_{j}=2$ e $a_{j+1}=2$, para todo $j \geq k+1$ então por (4.16) $b_{l}=a_{l}=2$ para todo $l \geq k+2$. Logo $b_{k} b_{k+1} b_{k+2} \ldots=10 \overline{2}$.

Determinando $c_{k} c_{k+1} \ldots$ se $\#(k-r)$ é par então pela equação (4.18)(a) $c_{k}=a_{k}^{\prime}=2$ como $a_{k}^{\prime}=2$ e $a_{k+1}^{\prime}=0$ então por (4.16) $c_{k+1}=a_{k+1}^{\prime}=0$. Agora, como $a_{j}^{\prime}=0=a_{j+1}^{\prime}=0$ para todo $j \geq k+1$ então por $(4.17)(a) c_{l}=2$, para todo $l \geq k+2$.

Assim, $\psi(t)=\left(b_{1} b_{2} \ldots b_{k-1} 10 \overline{2}\right)$ e $\psi\left(t^{\prime}\right)=\left(b_{1} b_{2} \ldots b_{k-1} 20 \overline{2}\right)$.

Portanto,

$f(t)=\lim _{n \longrightarrow \infty} g_{b_{1}} \circ g_{b_{2}} \circ \ldots \circ g_{b_{k-1}} \circ g_{1} \circ g_{0} \circ g_{2}^{n}\left(x_{0}\right) \quad$ e $f\left(t^{\prime}\right)=\lim _{n \longrightarrow \infty} g_{b_{1}} \circ g_{b_{2}} \circ \ldots \circ g_{b_{k-1}} \circ g_{2} \circ g_{0} \circ g_{2}^{n}\left(x_{0}\right)=f\left(t^{\prime}\right)$.

Este caso é igual ao Caso 2.

Se $a_{k}=1$ então pela equação (4.15) $b_{k}=1 . S e \#(k-r)$ é impar então por (4.18)(a) $b_{k+1}=a_{k+1}=2$. Como $a_{j}=2$ para todo $j \geq k+2$ então por (4.16) $b_{j}=2$ para todo $j \geq k+2$.

Determinando $c_{k} c_{k+1} \ldots$ se $\#(k-r)$ é impar então pela equação (4.18)(b) $c_{k}=2-a_{k}^{\prime}=$ $2-2=0$. Como $a_{k}^{\prime}=2$ e $a_{k+1}^{\prime}=0$ então por (4.16) $c_{k+1}=a_{k+1}^{\prime}=0$. Agora, como $a_{l}^{\prime}=0=a_{l+1}^{\prime}$ para todo $l \geq k+2$ então por $(4.17)(a) c_{l}=2$, para todo $l \geq k+2$. Assim, $\psi(t)=\left(b_{1} b_{2} \ldots b_{k-1} 1 \overline{2}\right)$ e $\psi\left(t^{\prime}\right)=\left(b_{1} b_{2} \ldots b_{k-1} 00 \overline{2}\right)$. 
Portanto,

$f(t)=\lim _{n \longrightarrow \infty} g_{b_{1}} \circ g_{b_{2}} \circ \ldots \circ g_{b_{k-1}} \circ g_{1} \circ g_{2}^{n}\left(x_{0}\right) \quad$ e $f\left(t^{\prime}\right)=\lim _{n \longrightarrow \infty} g_{b_{1}} \circ g_{b_{2}} \circ \ldots \circ g_{b_{k-1}} \circ g_{0}^{2} \circ g_{2}^{n}\left(x_{0}\right)=f\left(t^{\prime}\right)$.

Este caso é igual ao Caso 1.

3. Se $t=\left(a_{1} a_{2} \ldots a_{k-1} a_{k} a_{k+1} \ldots\right)$ onde $a_{1}=\ldots=a_{k-1}=1, a_{k}=1, a_{i}=2$ para todo $i \geq k+1$ e $t^{\prime}=\left(a_{1} a_{2} \ldots a_{k-1} a_{k} \ldots\right)$ onde $a_{1}=\ldots=a_{k-1}=1, a_{k}^{\prime}=2, a_{i}^{\prime}=0$ para todo $i \geq k+1$.

Vamos determinar $b_{k} b_{k+1} \ldots e c_{k} c_{k+1} \ldots$

Como $a_{k}=1$ então pela equação (4.15) $b_{k}=1$. Se \# $(k-r)$ é par então por (4.18)(b) $b_{k+1}=a_{k+1}=2$ e como $a_{l}=2=a_{l+1}$ para todo $l \geq k+2$ então $b_{l}=a_{l}=2$ para todo $l \geq k+2$.

Para determinar $c_{k} c_{k+1} \ldots$ se $\#(k-r)$ é par então pela equação (4.18)(b) $c_{k}=2-a_{k}^{\prime}=$ $2-2=0$. Como $a_{k}^{\prime}=2$ então por (4.16) $c_{k+1}=a_{k+1}^{\prime}=0$. Finalmente, como $a_{l}=a_{l+1}$ para todo $l \geq k+2$ então por (4.17)(a) $c_{l}^{\prime}=2$, para todo $l \geq k+2$.

Assim, $\psi(t)=\left(b_{1} b_{2} \ldots b_{k-1} 1 \overline{2}\right)$ e $\psi\left(t^{\prime}\right)=\left(b_{1} b_{2} \ldots b_{k-1} 00 \overline{2}\right)$ e conclui-se pelo Caso 1 que $f(t)=f\left(t^{\prime}\right)$.

Como $a_{k}=1$ então pela equação (4.15) $b_{k}=1$. Se \# $(k-r)$ é ímpar então por (4.18)(a) $b_{k+1}=2-a_{k+1}=2-2=0$. Se $a_{l}=2=a_{l+1}$ para todo $l \geq k+2$ então por (4.16) $b_{l}=a_{l}=2$ para todo $l \geq k+2$.

Para determinar $c_{k} c_{k+1} \ldots$ se \# $(k-r)$ é impar então pela equação (4.18)(a) $c_{k}=a_{k}^{\prime}=2$. Como $a_{k}^{\prime}=2$ então por (4.16) $c_{k+1}=a_{k+1}^{\prime}=0$. Agora, como $a_{l}^{\prime}=a_{l+1}^{\prime}=0$ para todo $l \geq k+2$ entấo por $(4.17)\left(\right.$ a) $c_{l}^{\prime}=2$, para todo $l \geq k+2$.

Assim, $\psi(t)=\left(b_{1} b_{2} \ldots b_{k-1} 10 \overline{2}\right)$ e $\psi\left(t^{\prime}\right)=\left(b_{1} b_{2} \ldots b_{k-1} 20 \overline{2}\right)$ e conclui-se pelo Caso 2 que $f(t)=f\left(t^{\prime}\right)$.

Portanto, em todos os casos analisados temos $f(t)=f\left(t^{\prime}\right)$, ou seja, $f$ está bem definida.

A injetividade e a continuidade estão demonstradas no Apêndice.

Teorema 4.1.4 A fronteira de $\mathcal{R}_{a}$ é homeomorfa ao círculo.

Pelo Teorema 4.1.1, temos que $f:[0,1] \longrightarrow \mathcal{R}_{\alpha-1}$ é contínua e bijetora.

Como $[0,1]$ é compacto, então para todo subconjunto fechado $F \subset[0,1]$, temos $\left(f^{-1}\right)^{-1}(F)=$ $f(F)$ é fechado em $\mathcal{R}_{\alpha-1}$ (pois é compacto). 
Logo $f^{-1}$ é contínua, portanto, $f=[0,1] \longrightarrow \mathcal{R}_{\alpha-1}$ é um homeomorfismo.

Seja

$$
\begin{array}{cl}
g_{1}:[0,1 / 6] & \longrightarrow \mathcal{R}_{\alpha-1} \\
t & \longmapsto
\end{array}
$$

Como $f$ é um homeomorfismo, então $g_{1}$ também é um homeomorfismo.

Da mesma maneira, usando o Lema 4.0.6 construimos as funções $g_{2}:[1 / 6,2 / 6] \longrightarrow \mathcal{R}_{-1}, g_{3}$ : $[2 / 6,3 / 6] \longrightarrow \mathcal{R}_{-\alpha}, g_{4}:[3 / 6,4 / 6] \longrightarrow \mathcal{R}_{-\alpha+1}, g_{5}:[4 / 6,5 / 6] \longrightarrow \mathcal{R}_{1}$ e $g_{6}:[5 / 6,1] \longrightarrow \mathcal{R}_{\alpha}$ onde $g_{i}, 2 \leq i \leq 6$ são homeomorfas.

Seja

$$
\begin{array}{ccc}
h: S^{1} \sim[0,1[ & \longrightarrow & \partial \mathcal{R} \\
t & \longmapsto & g_{i}(t), \quad \text { se } t \in[i-1 / 6, i / 6[
\end{array}
$$

Como as funções $g_{i}$ são homeomorfismos, temos que $h$ também o é.

Box

\subsection{Cálculo da dimensão de Hausdorff}

Como $\partial(\mathcal{R})$ é a reunião de seis curvas que são imagem de $\mathcal{R}_{\alpha-1}$ por uma transformação afim temos que $\operatorname{dim}_{H}\left(\mathcal{R}_{\alpha-1}\right)=\operatorname{dim}_{H}(\partial(\mathcal{R}))$. Pelo Lema 4.0.5 o conjunto $\mathcal{R}_{\alpha-1}=\cup_{i=0}^{2} g_{i}\left(\mathcal{R}_{\alpha-1}\right)$ é o conjunto invariante pelas semelhanças $g_{i}$. Uma cota superior da dimensão de Hausdorff dessa classe de compactos é dada pelo Teorema

Teorema 4.2.1 [11] Seja $A$ um conjunto de $\mathbb{C}$ tal que $A=\cup_{i=0}^{n} \varphi_{i}(A)$ é compacto e invariante pelas transformaçôes afins $\varphi_{i}$ de coeficientes $r_{i}$ (isto é , $\forall x, y \in \mathbb{C},\left|\varphi_{i}(x)-\varphi_{i}(y)\right|=r_{i}|x-y|$ ) então $\operatorname{dim}_{H}(A) \leq s$, onde s é o único número real que verifica $\sum_{i=0}^{n} r_{i}^{s}=1$.

Observação 4.2.2 Quando as $\varphi_{i}(A)$ se intersectam em pontos é conhecido que $\operatorname{dim}_{H}(A)=s$ (ver [11]).

Pelo Lema 4.0.5 temos $g_{0}\left(\mathcal{R}_{\alpha-1}\right) \cap g_{1}\left(\mathcal{R}_{\alpha-1}\right)$ e $g_{1}\left(\mathcal{R}_{\alpha-1}\right) \cap g_{2}\left(\mathcal{R}_{\alpha-1}\right)$ são pontos e $g_{0}\left(\mathcal{R}_{\alpha-1}\right) \cap$ $g_{2}\left(\mathcal{R}_{\alpha-1}\right)$ é vazio.

Para todo $x, y \in \mathcal{R}_{\alpha-1}$ temos:

$\left|g_{0}(x)-g_{0}(y)\right|=\left|\alpha^{2}\right||x-y|$. Logo $r_{0}=\left|\alpha^{2}\right|$.

$\left|g_{1}(x)-g_{1}(y)\right|=\left|\alpha^{3}\right||x-y|$. Então $r_{1}=\left|\alpha^{3}\right|$.

$\left|g_{2}(x)-g_{2}(y)\right|=\left|\alpha^{4}\right||x-y|$. Portanto, $r_{2}=\left|\alpha^{4}\right|$.

Consequentemente, $\operatorname{dim}_{H}\left(\mathcal{R}_{\alpha-1}\right)=s$, onde $s$ verifica

$$
|\alpha|^{2 s}+|\alpha|^{3 s}+|\alpha|^{4 s}=1
$$


Deduzimos que a $\operatorname{dim}_{H}\left(\mathcal{R}_{\alpha-1}\right)=\frac{\log \rho}{\log |\alpha|}=1.359337357$, onde $\rho$ é a raiz real máxima do polinômio $X^{4}+X^{3}+X^{2}-1=0$. 


\section{Capítulo}

Parametrização da fronteira de $\mathcal{F}$

Vamos considerar o Fractal de Rauzy clássico:

$$
\mathcal{F}=\mathcal{F}_{a}=\left\{\sum_{i=2}^{\infty} \varepsilon_{i} \alpha^{i} \mid \forall i \geq 2, \varepsilon_{i}=0,1, \ldots, a-1, \varepsilon_{i} \varepsilon_{i-1} \varepsilon_{i-2} \varepsilon_{i-3}<_{l e x}(a-1)(a-1) 01\right\} .
$$

O conjunto $\mathcal{F}$ foi estudado por vários matemáticos. Em particular ele é compacto, conexo, com interior simplesmente conexo e induz um azulejamento do plano módulo $\mathbb{Z}\left(\alpha^{-3}+\alpha^{-1}\right)+\mathbb{Z}\left(\alpha^{-2}+1\right)$ $(\operatorname{ver}([9]))$.

Proposição 5.0.3 A fronteira de $\mathcal{F}$ é $\partial \mathcal{F}=\bigcup_{v \in B} \mathcal{F} \cap(\mathcal{F}+v)$ onde $B$ é um conjunto finito contido em $\mathbb{Z}\left(\alpha^{-3}+\alpha^{-1}\right)+\mathbb{Z}\left(1+\alpha^{-2}\right)$ cuja cardinalidade é par e maior ou igual a 8 e $\left\{ \pm\left(\alpha^{-3}+\right.\right.$ $\left.\left.\alpha^{-1}\right), \pm \alpha^{-1}, \pm\left(1+\alpha^{-2}\right), \pm\left(\alpha+\alpha^{-2}\right)\right\} \subset B$.

Demonstração: Analogamente ao Teorema 2.1.8, item 1, provamos que $\partial \mathcal{F}=\bigcup_{v \in B} \mathcal{F} \cap$ $(\mathcal{F}+v)$, onde $B$ é um subconjunto finito de $\mathbb{Z}\left(\alpha^{-3}+\alpha^{-1}\right)+\mathbb{Z}\left(\alpha^{-2}+1\right)$ e de cardinalidade par.

Da próxima Proposição deduziremos que $\left\{ \pm\left(\alpha^{-3}+\alpha^{-1}\right), \pm \alpha^{-1}, \pm\left(1+\alpha^{-2}\right), \pm\left(\alpha+\alpha^{-2}\right)\right\} \subset B$.

Observação 5.0.4 Podemos provar usando o mesmo argumento que no Teorema 2.2.1 que B= $\left\{ \pm\left(\alpha^{-3}+\alpha^{-1}\right), \pm \alpha^{-1}, \pm\left(1+\alpha^{-2}\right), \pm\left(\alpha+\alpha^{-2}\right)\right\}$, isto é, $\mathcal{F}$ tem exatamente 8 vizinhos.

Como no fractal com restrições $\mathcal{R}$ temos para o fractal $\mathcal{F}$ o mesmo automato: 


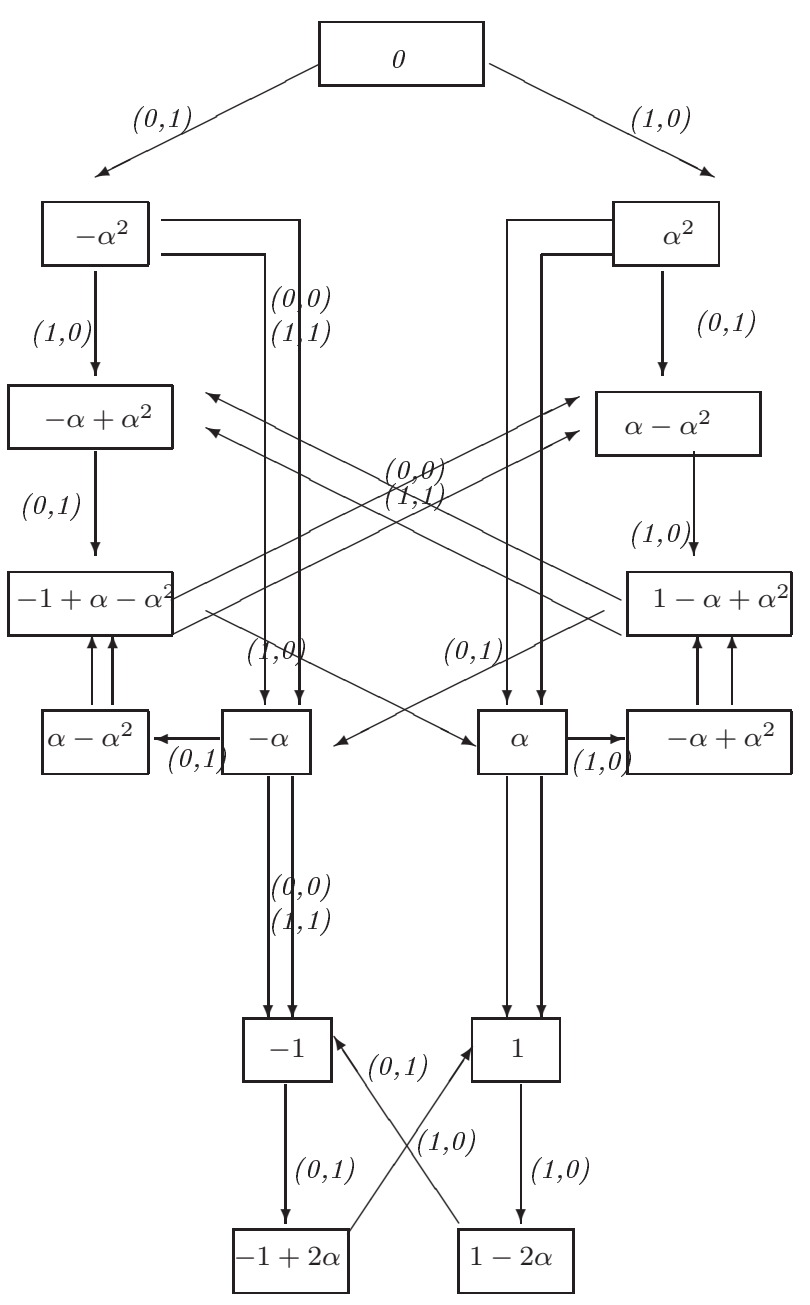




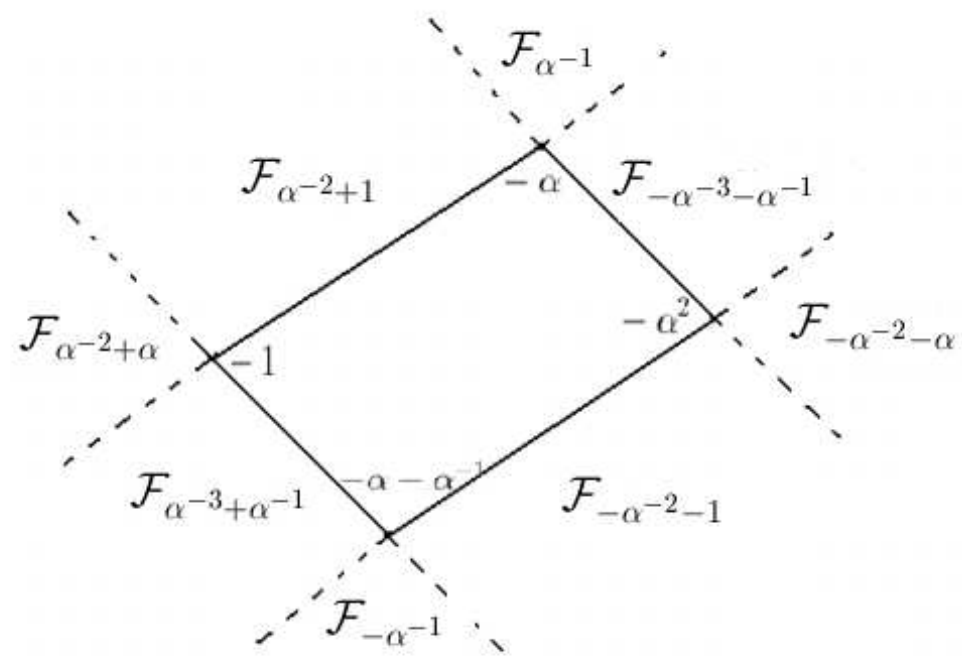

Figura 5.1: Fractal de Rauzy

Proposição 5.0.5 Seja $u \in\left\{ \pm\left(\alpha^{-3}+\alpha^{-1}\right), \pm \alpha^{-1}, \pm\left(1+\alpha^{-2}\right), \pm\left(\alpha^{-2}+\alpha\right)\right\}$ e $\mathcal{F}_{u}=\mathcal{F} \cap(\mathcal{F}+u)$ então as seguintes propriedades são verificadas:

1. $\mathcal{F}_{\alpha^{-3}+\alpha^{-1}} \cap \mathcal{F}_{1+\alpha^{-2}} \cap \mathcal{F}_{\alpha+\alpha^{-2}}=\{-1\}$;

2. $\mathcal{F}_{\alpha^{-1}} \cap \mathcal{F}_{1+\alpha^{-2}} \cap \mathcal{F}_{-\alpha^{-3}-\alpha^{-1}}^{\prime}=\{-\alpha\}$;

3. $\mathcal{F}_{-\alpha^{-3}-\alpha^{-1}}^{\prime} \cap \mathcal{F}_{-1-\alpha^{-2}}^{\prime} \cap \mathcal{F}_{-\alpha-\alpha^{-2}}^{\prime}=\left\{-\alpha^{2}\right\}$.

4. $\mathcal{F}_{\alpha^{-3}+\alpha^{-1}} \cap \mathcal{F}_{-\alpha^{-1}}^{\prime} \cap \mathcal{F}_{-1-\alpha^{-2}}^{\prime}=\left\{-\alpha-\alpha^{-1}\right\}$;

\section{Demonstração:}

1) Seja w' um elemento de $\mathcal{F} \cap\left(\mathcal{F}+\alpha^{-3}+\alpha^{-1}\right) \cap\left(\mathcal{F}+\alpha+\alpha^{-2}\right)$ entâo existem $x^{\prime}$, $y^{\prime}$ e $z^{\prime} \in \mathcal{F}$ tais que $x^{\prime}=\sum_{i=2}^{+\infty} a_{i}^{\prime} \alpha^{i}, y^{\prime}=\sum_{i=2}^{+\infty} b_{i}^{\prime} \alpha^{i}$ e $z^{\prime}=\sum_{i=2}^{+\infty} c_{i}^{\prime} \alpha^{i}$ e $w^{\prime}=\alpha-1+x^{\prime}=\alpha+\alpha^{-2}+y^{\prime}=z^{\prime}$. Como $\alpha-1=\alpha^{-3}+\alpha^{-1}$ então $r_{1}^{\prime}=(1,0)(0,0)(1,0)(0,0)(0,0)\left(a_{2}^{\prime}, c_{2}^{\prime}\right)\left(a_{3}^{\prime}, c_{3}^{\prime}\right) \ldots$ e para $\alpha+\alpha^{-2}$ temos $r_{2}^{\prime}=(1,0)(0,0)(0,0)(1,0)(0,1)(0,1)(1,0) t^{\prime} t^{\prime} t^{\prime} t^{\prime} t^{\prime} t^{\prime}$ onde $t^{\prime}=(1,0)(0,1)(0,1)(1,0)$ s $\tilde{a} o$ caminhos do autômato que começam no estado inicial.

Analisando o autômato obtemos

$$
\begin{aligned}
& r_{1}^{\prime}=(1,0)(0,0)(1,0)(0,0)(0,0)(0,1)(1,1)(1,0)(0,0)(0,1)(1,1)(1,0) \ldots e \\
& r_{2}^{\prime}=(1,0)(0,0)(0,0)(1,0)(0,1)(0,1)(1,0) t^{\prime} t^{\prime} t^{\prime} t^{\prime} t^{\prime} \ldots \text { onde } t^{\prime}=(1,0)(0,1)(0,1)(1,0) .
\end{aligned}
$$


Assim

$$
w^{\prime}=\alpha-1+x^{\prime}=\alpha^{-3}+\alpha^{-1}+\sum_{i=1}^{+\infty}\left(\alpha^{4 i-1}+\alpha^{4 i}\right)
$$

Por outro lado

$$
w^{\prime}=z^{\prime}=\alpha^{2}+\alpha^{3}+\sum_{i=1}^{+\infty}\left(\alpha^{4 i+2}+\alpha^{4 i+3}\right)
$$

$e$

$$
w^{\prime}=\alpha+\alpha^{-2}+y^{\prime}=\sum_{i=2}^{+\infty}\left(\alpha^{4 i-2}+\alpha^{4 i-1}\right)
$$

Das equações (5.1) à (5.3) obtemos, por exemplo (usando a equação (5.2)):

$$
w^{\prime}=\alpha^{2}+\alpha^{3}+\left(\alpha^{6}+\alpha^{7}\right)+\left(\alpha^{10}+\alpha^{11}\right)+\left(\alpha^{14}+\alpha^{15}\right)+\ldots
$$

Como $\alpha^{8}=\alpha^{7}+\alpha^{6}+\alpha^{4}$ então

$$
\begin{array}{ccc}
w^{\prime} & = & \alpha^{2}+\alpha^{3}-\alpha^{4}+\left(\alpha^{4}+\alpha^{6}+\alpha^{7}\right)+\left(\alpha^{10}+\alpha^{11}\right)+\ldots \\
& = & \alpha^{2}+\alpha^{3}-\alpha^{3}-\alpha^{2}-1+0=-1
\end{array}
$$

Portanto, $w^{\prime}=-1$.

Seja $w^{\prime \prime} \in \mathcal{F} \cap\left(\mathcal{F}+\alpha^{-3}+\alpha^{-1}\right) \cap\left(\mathcal{F}+1+\alpha^{2}\right)$ então existem $x^{\prime \prime}, y^{\prime \prime}$ e $z^{\prime \prime} \in \mathcal{F}$ tais que $x^{\prime \prime}=\sum_{i=2}^{+\infty} a_{i}^{\prime \prime} \alpha^{i}, y^{\prime \prime}=\sum_{i=2}^{+\infty} b_{i}^{\prime \prime} \alpha^{i}$ e $z^{\prime \prime}=\sum_{i=2}^{+\infty} c_{i}^{\prime \prime} \alpha^{i}$ e $w^{\prime \prime}=\alpha-1+x^{\prime \prime}=1+\alpha^{-2}+y^{\prime \prime}=z^{\prime \prime}$.

Como $\alpha-1=\alpha^{-3}+\alpha^{-1}$ então $r_{1}^{\prime \prime}=(1,0)(0,0)(1,0)(0,0)(0,0)\left(a_{2}^{\prime \prime}, c_{2}^{\prime \prime}\right)\left(a_{3}^{\prime \prime}, c_{3}^{\prime \prime}\right) \ldots$ e para $1+\alpha^{-2}$ temos $r_{2}^{\prime \prime}=(1,0)(0,0)(1,0)(0,0)(1,1)\left(a_{3}^{\prime \prime}, b_{3}^{\prime \prime}\right)\left(a_{4}^{\prime \prime}, b_{4}^{\prime \prime}\right) \ldots$ são caminhos do autômato que começam no estado inicial.

Analisando o autômato obtemos:

$$
\begin{array}{lcc}
r_{1}^{\prime \prime} & = & (1,0)(0,0)(1,0)(0,0)(0,0)(0,1)(1,1)(1,0)(0,0)(0,1)(1,1)(1,0) \ldots e \\
r_{2}^{\prime \prime} & = & (1,0)(0,0)(1,0)(0,0)(1,1)(0,1)(0,0)(1,0)(1,1)(0,1)(0,0)(1,0)(1,1)(0,1)(0,0)(1,0) \ldots
\end{array}
$$

Assim

$$
w^{\prime \prime}=\alpha-1+x^{\prime \prime}=\alpha^{-3}+\alpha^{-1}+\sum_{i=1}^{+\infty}\left(\alpha^{4 i-1}+\alpha^{4 i}\right)
$$

Por outro lado

$$
w^{\prime \prime}=z^{\prime \prime}=\alpha^{2}+\alpha^{3}+\sum_{i=1}^{+\infty}\left(\alpha^{4 i+2}+\alpha^{4 i+3}\right)
$$


e

$$
w^{\prime \prime}=1+\alpha^{-2}+y^{\prime \prime}=1+\alpha^{-2}+\alpha^{2}+\sum_{i=1}^{+\infty}\left(\alpha^{4 i+1}+\alpha^{4 i+2}\right)
$$

Das equações (5.4) à (5.6) obtemos, por exemplo (usando a equação (5.6)):

$$
\begin{array}{rlc}
w^{\prime \prime} & = & 1+\alpha^{-2}+y^{\prime \prime}=1+\alpha^{-2}+\alpha^{2}+\sum_{i=1}^{+\infty}\left(\alpha^{4 i+1}+\alpha^{4 i+2}\right) \\
& = & 1+\alpha^{-2}+\alpha^{2}-\alpha^{3}+\left(\alpha^{3}+\alpha^{5}+\alpha^{6}\right)+\left(\alpha^{9}+\alpha^{10}\right)+\ldots \\
& = & \alpha^{-2}-2 \alpha^{-1}+\alpha^{-3}=\alpha^{-2}-1-\alpha^{-2}
\end{array}
$$

Portanto, $w^{\prime \prime}=-1$ e assim $\mathcal{F} \cap\left(\mathcal{F}+\left(\alpha^{-3}+\alpha^{-1}\right)\right) \cap\left(\mathcal{F}+\left(1+\alpha^{-2}\right)\right) \cap\left(\mathcal{F}+\left(\alpha+\alpha^{-2}\right)\right)=\{-1\}$.

Analogamente provamos os outros itens.

De fato, mostraremos que $\mathcal{F}_{\alpha^{-3}+\alpha^{-1}}=\mathcal{F} \cap\left(\mathcal{F}+\alpha^{-3}+\alpha^{-1}\right)$ é homeomorfo a um segmento de reta. Sejam $a, b \in \mathcal{F}_{\alpha^{-3}+\alpha^{-1}}$. Se denotarmos por $I(a, b)$ o segmento em $\mathcal{F}_{\alpha^{-3}+\alpha^{-1}}$ ligando a e b, em virtude do Lema 5.0.6 teremos que

$$
\begin{aligned}
& h_{0}\left(\mathcal{F}_{\alpha^{-3}+\alpha^{-1}}\right)=h_{0}\left(\mathcal{F}_{\alpha-1}\right)=I\left(-\alpha-\alpha^{-1},-1-\alpha^{3}\right)=I\left(h_{0}(-1), h_{0}\left(-\alpha-\alpha^{-1}\right)\right) \\
& h_{1}\left(\mathcal{F}_{\alpha^{-3}+\alpha^{-1}}\right)=h_{1}\left(\mathcal{F}_{\alpha-1}\right)=I\left(-1-\alpha^{3},-\left(1+\alpha^{2}+\alpha^{4}\right)\right)=I\left(h_{1}(-1), h_{1}\left(-\alpha-\alpha^{-1}\right)\right) \\
& h_{2}\left(\mathcal{F}_{\alpha^{-3}+\alpha^{-1}}\right)=h_{2}\left(\mathcal{F}_{\alpha-1}\right)=I\left(-\left(1+\alpha^{2}+\alpha^{4}\right),-1\right)=I\left(h_{2}\left(-\alpha-\alpha^{-1}\right), h_{2}(-1)\right) .
\end{aligned}
$$

Lema 5.0.6 Sejam $h_{0}(z)=\alpha-1+\alpha^{2} z, h_{1}(z)=-1+\alpha^{3} z$ e $h_{2}(z)=\alpha^{2}+\alpha^{3}+\alpha^{4} z$, temos que $\mathcal{F}_{\alpha-1}$ verifica as seguintes propriedades:

1. $\mathcal{F}_{\alpha-1}=h_{0}\left(\mathcal{F}_{\alpha-1}\right) \cup h_{1}\left(\mathcal{F}_{\alpha-1}\right) \cup h_{2}\left(\mathcal{F}_{\alpha-1}\right)$;

2. $h_{1}\left(\mathcal{F}_{\alpha-1}\right) \cap h_{2}\left(\mathcal{F}_{\alpha-1}\right)=\left\{-\left(1+\alpha^{2}+\alpha^{4}\right)\right\}=\left\{h_{2}\left(-\alpha-\alpha^{-1}\right)\right\}$;

3. $h_{1}\left(\mathcal{F}_{\alpha-1}\right) \cap h_{0}\left(\mathcal{F}_{\alpha-1}\right)=\left\{-\left(1+\alpha^{3}\right)\right\}=\left\{h_{1}(-1)\right\}$;

4. $h_{0}\left(\mathcal{F}_{\alpha-1}\right) \cap h_{2}\left(\mathcal{F}_{\alpha-1}\right)=\emptyset$.

Demonstração: $\quad$ Vamos mostrar que $h_{i}\left(\mathcal{F}_{\alpha-1}\right) \subset \mathcal{F}_{\alpha-1}, \forall i \in\{0,1,2\}$.

1. Temos que $h_{0}\left(\mathcal{F}_{\alpha-1}=\left(\alpha^{-3}+\alpha^{-1}+\alpha^{2} \mathcal{F}\right) \cap\left(\alpha^{-3}+\alpha^{-1}+\alpha^{2} \mathcal{F}\right)=\left(\alpha^{-3}+\alpha^{-1}+\alpha^{2} \mathcal{F}\right) \cap\left(\alpha^{2}+\right.\right.$ $\left.\alpha^{2} \mathcal{F}\right)=\left((\alpha-1)+\alpha^{2} \mathcal{F}\right) \cap\left(\alpha^{2}+\alpha^{2} \mathcal{F}\right) \subset \mathcal{F}_{\alpha-1}$.

$h_{2}\left(\mathcal{F}_{\alpha-1}\right)=\left(\alpha^{2}+\alpha^{3}+\alpha^{4} \mathcal{F}\right) \cap\left(\alpha+\alpha^{2}+2 \alpha^{3}+\alpha^{4} \mathcal{F}\right)$. Por outro lado, $\alpha+\alpha^{2}+2 \alpha^{3}=$ $(\alpha-1)+\alpha^{3}+\alpha^{4}+\alpha^{4} \mathcal{F}$. Logo, $h_{2}\left(\mathcal{F}_{\alpha-1}\right)=\left(\alpha^{2}+\alpha^{3}+\alpha^{4} \mathcal{F}\right) \cap\left((\alpha-1)+\alpha^{3}+\alpha^{4}+\alpha^{4} \mathcal{F}\right) \subset \mathcal{F}_{\alpha-1}$. Seja $x \in \mathcal{F}_{\alpha-1}$ então $x=\alpha-1+\sum_{i=2}^{+\infty} \varepsilon_{i} \alpha^{i}=\sum_{i=2}^{+\infty} \varepsilon_{i}^{\prime} \alpha^{i}$, onde $\left(\varepsilon_{i}\right)_{i \geq 2}, \quad\left(\varepsilon_{i}^{\prime}\right)_{i \geq 2} \in \mathcal{D}^{\infty}$. 
Como $\alpha-1=\alpha^{-3}+\alpha^{-1}$ então

$$
(1,0)(0,0)(1,0)(0,0)(0,0)\left(\varepsilon_{2}, \varepsilon_{2}^{\prime}\right)\left(\varepsilon_{3}, \varepsilon_{3}^{\prime}\right)\left(\varepsilon_{4}, \varepsilon_{4}^{\prime}\right) \ldots
$$

é um caminho no autômato começando do estado inicial.

Portanto, temos duas possibilidades. Considerando a primeira: $\left(\varepsilon_{2}, \varepsilon_{2}^{\prime}\right)\left(\varepsilon_{3}, \varepsilon_{3}^{\prime}\right)\left(\varepsilon_{4}, \varepsilon_{4}^{\prime}\right)=(0,1)(0,0)(1,0)$.

Logo

$$
x=\alpha^{-3}+\alpha^{-1}+\alpha^{4}+\sum_{i=5}^{+\infty} \varepsilon_{i} \alpha^{i}=\alpha^{2}+\sum_{i=5}^{+\infty} \varepsilon_{i}^{\prime} \alpha^{i} .
$$

Assim, $x=\alpha^{-3}+\alpha^{-1}+\alpha^{4}+\alpha^{3} z=\alpha^{2}+\alpha^{3} z^{\prime}$, onde $z, z^{\prime} \in \mathcal{F}$. Então, $h_{1}(x)=-1+\alpha^{3}\left(\alpha^{-3}+\right.$ $\left.\alpha^{-1}+\alpha^{4}+\alpha^{3} z\right)$ e $h_{1}(x)=-1+\alpha^{3}\left(\alpha^{2}+\alpha^{3} z^{\prime}\right)$. Logo, $h_{1}(x)=-1+1+\alpha^{2}+\alpha^{7}+\alpha^{6} z=$ $\alpha^{2}+\alpha^{7}+\alpha^{6} z \in \mathcal{F}$ e $h_{1}(x)=-1+\alpha^{5}+\alpha^{6} z^{\prime}=(\alpha-1)+\alpha^{3}+\alpha^{4}+\alpha^{6} z^{\prime} \in \mathcal{F}+(\alpha-1)$.

Portanto, $h_{1}\left(\mathcal{F}_{\alpha-1} \subset \mathcal{F}_{\alpha-1}\right.$.

A segunda possibilidade é $:\left(\varepsilon_{2}, \varepsilon_{2}^{\prime}\right)\left(\varepsilon_{3}, \varepsilon_{3}^{\prime}\right)\left(\varepsilon_{4}, \varepsilon_{4}^{\prime}\right)=(0,1)(1,1)(1,0)$. Logo

$$
x=\alpha^{-3}+\alpha^{-1}+\alpha^{3}+\alpha^{4}+\sum_{i=5}^{+\infty} \varepsilon_{i} \alpha^{i}=\alpha^{2}+\alpha^{3}+\sum_{i=5}^{+\infty} \varepsilon_{i}^{\prime} \alpha^{i} .
$$

Assim, $x=\alpha^{-3}+\alpha^{-1}+\alpha^{3}+\alpha^{4}+\alpha^{3} \widehat{z}=\alpha^{2}+\alpha^{3}+\alpha^{3} \widetilde{z}$, onde $\widehat{z}, \widetilde{z} \in \mathcal{F}$.

Então, $h_{1}(x)=-1+\alpha^{3}\left(\alpha^{-3}+\alpha^{-1}+\alpha^{3}+\alpha^{4}+\alpha^{3} \widehat{z}\right)$ e $h_{1}(x)=-1+\alpha^{3}\left(\alpha^{2}+\alpha^{3}+\alpha^{3} \widetilde{z}\right)$. Logo, $h_{1}(x)=\alpha^{2}+\alpha^{3}+\alpha^{4}+\alpha^{3} \widehat{z} \in \mathcal{F}$ e $h_{1}(x)=-1+\alpha^{5}+\alpha^{6}+\alpha^{6} \widetilde{z}=-1+\alpha^{4}+\alpha^{3}+\alpha+\alpha^{5}+\alpha^{6}+\alpha^{6} \widetilde{z}=$ $(\alpha-1)+\alpha^{4}+\alpha^{7}+\alpha^{6} \widetilde{z} \in \mathcal{F}+(\alpha-1)$.

Portanto, $h_{1}\left(\mathcal{F}_{\alpha-1}\right) \subset \mathcal{F}_{\alpha-1}$.

Por outro lado, seja z um elemento de $\mathcal{F}_{\alpha-1}$. Devido ao automato, temos três casos:

(a) $z=\alpha-1+\alpha^{3}+\alpha^{4}+\alpha^{3} t_{0}=\alpha^{2}+\alpha^{3} t_{0}^{\prime}$ onde $t_{0}, t_{0}^{\prime} \in \mathcal{F}$. Neste caso $h_{0}^{-1}(z)=\alpha^{2}+t_{0}=$ $(\alpha-1)+t_{0}^{\prime} \in \mathcal{F} \cap(\mathcal{F}+\alpha-1)$, dai $z \in h_{0}\left(\mathcal{F}_{\alpha-1}\right)$.

(b) $z=\alpha-1+\alpha^{3}+\alpha^{4}+\alpha^{4} t_{1}=\alpha^{2}+\alpha^{3}+\alpha^{4} t_{1}^{\prime}$ onde $t_{1}, t_{1}^{\prime} \in \mathcal{F}$. Neste caso, $h_{1}^{-1}(z)=$ $(\alpha-1)+t_{1}=t_{1}^{\prime} \in \mathcal{F}_{\alpha-1}$, dai $z \in h_{1}\left(\mathcal{F}_{\alpha-1}\right)$.

(c) $z=\alpha-1+\alpha^{4}+\alpha^{2} t_{2}=\alpha^{2}+\alpha^{2} t_{2}^{\prime}$ onde $t_{2}, t_{2}^{\prime} \in \mathcal{F}$. Neste caso, $h_{2}^{-1}(z)=\alpha^{2}+t_{2}=\alpha-1+t_{2}^{\prime}$. Portanto, $X=\mathcal{F}_{\alpha-1}=h_{0}\left(\mathcal{F}_{\alpha-1}\right) \cup h_{1}\left(\mathcal{F}_{\alpha-1}\right) \cup h_{2}\left(\mathcal{F}_{\alpha-1}\right)$.

2. Suponha $\gamma \in h_{1}\left(\mathcal{F}_{\alpha-1}\right) \cap h_{2}\left(\mathcal{F}_{\alpha-1}\right)$. Então existem $y, y_{1} \in \mathcal{F}_{\alpha-1}$ tais que $\gamma=-1+\alpha^{3} y=$ $\alpha^{2}+\alpha^{3}+\alpha^{4} y_{1}$. E assim, temos $y=\alpha+\alpha y_{1}$.

Como $\alpha^{2}=\alpha+1+\alpha^{-2}$ podemos reescrever $y=-1-\alpha^{-2}+\alpha^{2}+\alpha y_{1}$. 
Os elementos de $\mathcal{F}_{\alpha-1}$ podem ser escritos de duas maneiras, considerando a primeira $y_{1}=$ $\alpha^{-3}+\alpha^{-1}+z^{\prime}, z^{\prime} \in \mathcal{F}$, temos $y=\alpha^{2}+\alpha z^{\prime}=\alpha^{2}+\omega$, onde $w \in \mathcal{F}$.

Agora considerando a segunda forma, temos que se $y_{1}=\alpha^{2}+z^{\prime \prime}, z^{\prime \prime} \in \mathcal{F}$, então

$$
y=-1-\alpha^{-2}+\alpha^{2}+\alpha^{3}+\alpha z^{\prime \prime} . \text { Logo } y \in \mathcal{F} \cap\left(\mathcal{F}-1-\alpha^{-2}\right) .
$$

Por outro lado, temos que

$$
-1+\alpha^{3} y=\alpha^{2}+\alpha^{3}+\alpha^{4} y_{1} . \text { Assim }-1-\alpha^{2}-\alpha^{3}+\alpha^{3} y=\alpha^{4} y_{1} \text { então } y_{1}=-1+\alpha^{-1} y \text {. }
$$

Temos que y pode ser escrito de duas maneiras: considerando a primeira $y=\alpha^{2}+w_{1}, w_{1} \in \mathcal{F}$ temos $y_{1}=-1+\alpha+w_{1} \alpha^{-1}$, onde $w_{1} \alpha^{-1} \in \mathcal{F}$ (pois, analisando as possibilidades para $w_{1}=\alpha^{3}+a_{4} \alpha^{4}+a_{5} \alpha^{5}+a_{6} \alpha^{6}+\ldots$, onde $a_{i} \in\{0,1\}, \forall i \geq 4$, temos que se $w_{1}=\alpha^{3}+\alpha^{4}+$ $\alpha^{5}+\alpha^{7}+\alpha^{9}+\ldots$ então $w_{1} \alpha^{-1}=\alpha^{2}+\alpha^{3}+\alpha^{4}+\alpha^{6}+\ldots \in \mathcal{F}$, ou se $w_{1}=\alpha^{3}+\alpha^{5}+\alpha^{7}+\alpha^{9}+\ldots$ então $w_{1} \alpha^{-1}=\alpha^{2}+\alpha^{4}+\alpha^{6}+\alpha^{8}+\ldots \in \mathcal{F}$ ou finalmente, se $w_{1}=\alpha^{3}+\alpha^{4}+\alpha^{6}+\alpha^{8}+\ldots$ então $\left.w_{1} \alpha^{-1}=\alpha^{2}+\alpha^{3}+\alpha^{5}+\alpha^{7}+\ldots \in \mathcal{F}\right), \operatorname{logo}$

$$
y_{1} \in \mathcal{F} \cap(\mathcal{F}+\alpha-1)=\mathcal{F} \cap\left(\mathcal{F}+\alpha^{-3}+\alpha^{-1}\right) .
$$

Agora, seja $y=\alpha^{-3}+\alpha^{-1}+w_{2}$, com $w_{2} \in \mathcal{F}$ temos

$$
y_{1}=-1+\alpha^{-4}+\alpha^{-2}+w_{2} \alpha^{-1} \operatorname{logo}-\alpha^{-1}+w_{2} \alpha^{-1}, w_{2} \alpha^{-1} \in \mathcal{F} \text { assim } y_{1} \in \mathcal{F} \cap\left(\mathcal{F}-\alpha^{-1}\right)
$$

De (5.7), (5.8) e (5.9) segue que $y_{1}=-\alpha-\alpha^{-1}=y$ e dai

$$
\gamma=-1+\alpha^{3} y=\alpha^{2}+\alpha^{3}+\alpha^{4} y_{1}=-1-\alpha^{2}-\alpha^{4} .
$$

3. Suponha $\tilde{\gamma} \in h_{1}\left(\mathcal{F}_{\alpha-1}\right) \cap h_{0}\left(\mathcal{F}_{\alpha-1}\right)$ Então, temos que $\tilde{\gamma}=-1+\alpha^{3} y_{2}=\alpha-1+\alpha^{2} y_{3}$ logo $y_{3}=-\alpha^{-1}+\alpha y_{2}$.

Como anteriormente, podemos escrever $y_{2}$ de duas maneiras, se considerarmos $y_{2}=\alpha^{2}+$ $z_{1}, z_{1} \in \mathcal{F}$ temos $y_{3}=-\alpha^{-1}+\alpha^{3}+\alpha z_{1}$ então $y_{3} \in \mathcal{F} \cap\left(\mathcal{F}+\alpha^{-3}+\alpha^{-1}\right) \cap\left(\mathcal{F}-\alpha^{-1}\right)$. Agora, se $y_{2}=\alpha^{-3}+\alpha^{-1}+z_{2}$, onde $z_{2} \in \mathcal{F}$, temos $y_{3}=\alpha-1+\alpha z_{2}$ logo $y_{3} \in \mathcal{F}+\alpha-1$. Por outro lado, $-1+\alpha^{3} y_{2}=\alpha-1+\alpha^{2} y_{3}$ então $\alpha^{3} y_{2}=\alpha+\alpha^{2} y_{3}$ logo $y_{2}=\alpha^{-2}+\alpha^{-1} y_{3}$. Podemos escrever $y_{3}$ de duas maneiras. Considerando a primeira $y_{3}=\alpha^{2}+w_{3}$, com $w_{3} \in \mathcal{F}$ 
então

$$
y_{2}=\alpha^{-2}+\alpha^{-1}\left(\alpha^{2}+w_{3}\right)=\alpha^{-2}+\alpha+w_{3} \alpha^{-1}, w_{3} \alpha^{-1} \in \mathcal{F} \text { então } y_{2} \in \mathcal{F} \cap\left(\mathcal{F}+\alpha^{-2}+\alpha\right)
$$

Agora, se considerarmos $y_{3}=\alpha^{-3}+\alpha^{-1}+w_{4}, w_{4} \in \mathcal{F}$, temos

$y_{2}=\alpha^{-2}+\alpha^{-1}\left(\alpha^{-3}+\alpha^{-1}+w_{4}\right)=\alpha-1+w_{4} \alpha^{-1}, w_{4} \alpha^{-1} \in \mathcal{F}$ então $y_{2} \in \mathcal{F} \cap(\mathcal{F}+\alpha-1)$

Assim, $y_{2} \in \mathcal{F} \cap(\mathcal{F}+\alpha-1) \cap\left(\mathcal{F}+\alpha^{-2}+\alpha\right)$, portanto, usando o autômato duplo temos que $y_{2}=-1$ e consequentemente, $\tilde{\gamma}=-1+\alpha^{3} y_{2}=\alpha-1+\alpha^{2} y_{3}$ então $\tilde{\gamma}=-1-\alpha^{3}$.

4. Seja $\hat{\gamma} \in h_{2}\left(\mathcal{F}_{\alpha-1}\right) \cap h_{0}\left(\mathcal{F}_{\alpha-1}\right)$. Logo $\hat{\gamma}=\alpha^{2}+\alpha^{3}+\alpha^{4} y_{4}=\alpha-1+\alpha^{2} y_{5}$ com $y_{4}, y_{5} \in(\mathcal{F}+\alpha-1)$. Temos

$$
y_{5}=-\alpha^{-1}+\alpha^{2}+\alpha^{2} y_{4}
$$

Podemos escrever $y_{4}$ de duas maneiras, considerando a primeira $y_{4}=\alpha^{2}+z_{3}, z_{3} \in \mathcal{F}$. Daí,

$$
y_{5}=-\alpha^{-1}+\alpha^{2}+\alpha^{4}+\alpha^{2} z_{3} \text { então } y_{5} \in \mathcal{F} \cap(\mathcal{F}-\alpha-1)
$$

Também podemos escrever $y_{5}=\alpha^{-3}+\alpha^{-1}+z_{4}, z_{4} \in \mathcal{F}$ e temos

$$
y_{5}=\alpha+\alpha^{2}+\alpha^{2} z_{4} \operatorname{logo} y_{5} \in \mathcal{F} \cap(\mathcal{F}+\alpha)
$$

De (5.12), (5.13) e (5.14) segue que $y_{5} \in \mathcal{F} \cap(\mathcal{F}+\alpha-1) \cap\left(\mathcal{F}-\alpha^{-1}\right) \cap(\mathcal{F}+\alpha)$ que pela Proposição 5.0 .5 é vazio.

\subsection{Parametrização de $\mathcal{F}_{\alpha-1}$}

Analogamente ao que foi feito no Capítulo 4, será definida uma aplicação $f$ a qual parametrizará a fronteira de $\mathcal{F}$. Veremos que esta aplicação está bem definida, contínua e bijetora.

Seja z um elemento de $\mathcal{F}_{\alpha-1}$. Pelo Lema 5.0.6, existem $z_{1} \in \mathcal{F}_{\alpha-1}$ e a elemento de $A=\{0,1,2\}$ tais que $z=h_{a_{1}}\left(z_{1}\right)$. Continuando assim construímos uma sequência $\left(a_{n}\right)_{n \geq 1}$ em $\{0,1,2\}^{\mathbb{N}}$ e uma sequência $\left(z_{n}\right)_{n \geq 1}$ em $X$, tais que para todo $n$ :

$$
z=h_{a_{1}} \circ h_{a_{2}} \circ \ldots \circ h_{a_{n}}\left(z_{n}\right)
$$

Como as funções $h_{i}$ são contrações, então para todo $x \in \mathcal{F}_{\alpha-1}$, a sequência $h_{a_{1}} \circ h_{a_{2}} \circ \ldots \circ h_{a_{n}}(x)$ 
converge para z quando $n$ tende a infinito.

Seja

$$
\begin{aligned}
& \psi: A^{\mathbb{N}} \longrightarrow A^{\mathbb{N}} \\
& a_{1} a_{2} \ldots \longmapsto b_{1} b_{2} \ldots
\end{aligned}
$$

definida como segue.

Temos $b_{1}=a_{1}$.

Seja $k \geq 2$, definimos $b_{k}$ da seguinte maneira:

$$
\text { Se } a_{k}=1 \text { então } b_{k}=1 \text {; }
$$

$$
\text { Se } a_{k} \neq 1 \text { e } \quad a_{k-1}=2 \quad \text { então } \quad b_{k}=a_{k}
$$

$$
\text { Se } a_{k} \neq 1 \text { e } \quad a_{k-1}=0 \quad \text { então } \quad \begin{aligned}
a) b_{k} & =2 \text { se } a_{k}=0 ; \\
b) b_{k} & =0 \text { se } a_{k}=2 .
\end{aligned}
$$

Se $a_{k} \neq 1$ e $a_{k-1}=1$, seja $r=\min \left\{1 \leq i \leq k-1, a_{i}=a_{i+1}=\ldots=a_{k-1}=1\right\}$ :

- (Se $r>1$ e $\left.a_{r-1}=2\right)$ ou $r=1$ então

$$
\left\{\begin{array}{c}
a) b_{k}=a_{k}, \quad \text { se \#(k-r) é par } \\
b) b_{k}=2-a_{k}, \quad \text { se \# }(k-r) \quad \text { é ímpar }
\end{array}\right.
$$

onde \# $(k-r)$ é o número de algarismos 1 após um algarismo 0 ou 2.

- $\left(\right.$ Se $r>1$ e $\left.a_{r-1}=0\right)$ então

$$
\left\{\begin{array}{c}
a) b_{k}=a_{k}, \quad \text { se \#(k-r) é ímpar } \\
b) b_{k}=2-a_{k}, \quad \text { se \# }(k-r) \quad \text { é par }
\end{array}\right.
$$

Fixemos $x_{0} \in \mathcal{F}_{\alpha-1}$ e definimos $f:[0,1] \longrightarrow \mathcal{F}_{\alpha-1}$ a correspondência definida da seguinte maneira: se $t=\sum_{i=1}^{\infty} a_{i} 3^{-i}$ onde $\left(a_{i}\right)_{i \geq 0} \in A^{\mathbb{N}}$, então $f(t)=\lim _{n \longrightarrow \infty} h_{b_{1}} \circ \ldots \circ h_{b_{n}}\left(x_{0}\right)$ onde $b_{1} b_{2} \ldots=$ $\psi\left(a_{1} a_{2} \ldots\right)$.

Daqui pra frente, supomos que se $t$ e $t^{\prime}$ pertencem a $[0,1]$ então $t=\sum_{i=1}^{\infty} a_{i} 3^{-i}$ e $t^{\prime}=\sum_{i=1}^{\infty} b_{i} 3^{-i}$ onde $a_{i}$ e $b_{i}$ são elementos de $\{0,1,2\}$ tais que $a_{i}=b_{i}$ para $i<k$ e $a_{k}<b_{k}$ onde $k \in \mathbb{N}$. Supomos que $f(t)=\lim _{n \longrightarrow \infty} h_{c_{1}} \circ \ldots \circ h_{c_{n}}\left(x_{0}\right)$ e $f\left(t^{\prime}\right)=\lim _{n \longrightarrow \infty} h_{d_{1}} \circ \ldots \circ h_{d_{n}}\left(x_{0}\right)$ onde $c_{1} c_{2} \ldots=\psi\left(a_{1} a_{2} \ldots\right)$ e $d_{1} d_{2} \ldots=\psi\left(b_{1} b_{2} \ldots\right)$. 
Proposição 5.1.1: Temos que $f(0)=\lim _{n \longrightarrow \infty} h_{2}\left(h_{0}^{n}(z)\right)=-\alpha-\alpha^{-1}$ e $f(1)=\lim _{n \longrightarrow \infty} h_{0}^{n}(z)=-1$. Temos que $f$ está bem definida, é bijetora e contínua.

A demonstração desta proposição é análoga à que foi feita no capítulo 4 para o fractal $\mathcal{R}$.

\subsection{Cálculo da dimensão de Hausdorff}

Como no caso do fractal com restrições temos $\partial(\mathcal{F})$ é a reunião de oito curvas, onde cada uma delas é a imagem de $\mathcal{F}_{\alpha-1}$ por uma transformação afim. Em particular $\operatorname{dim}_{H}\left(\mathcal{F}_{\alpha-1}\right)=\operatorname{dim}_{H}(\partial(\mathcal{F}))$. Pelo Lema 5.0.6 temos que $\mathcal{F}_{\alpha-1}=\cup_{i=0}^{2} h_{i}\left(\mathcal{F}_{\alpha-1}\right)$, isto é, $\mathcal{F}_{\alpha-1}$ é o conjunto maximal invariante pelas semelhanças $h_{i}$.

Usando novamente o Teorema 4.2.1 temos

$$
|\alpha|^{2 s}+|\alpha|^{3 s}+|\alpha|^{4 s}=1 .
$$

E consequentemente a mesma dimensão de Hausdorff, a qual é $\operatorname{dim}_{H}\left(\mathcal{F}_{\alpha-1}\right)=\frac{\log \rho}{\log |\alpha|}=1.359337357$, onde $\rho$ é a raiz real máxima do polinômio $X^{4}+X^{3}+X^{2}-1=0$. 


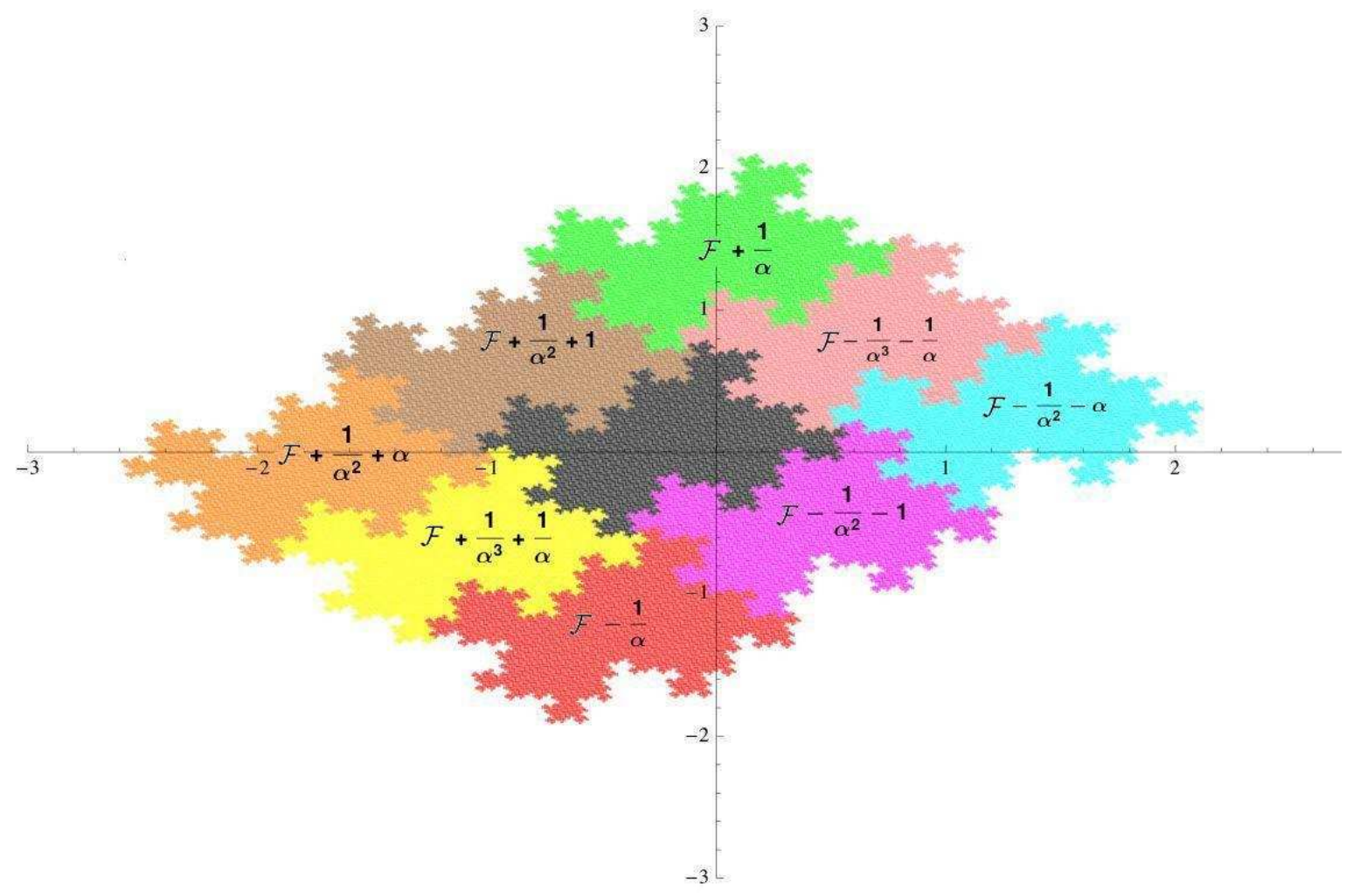

Figura 5.2: Fractal de Rauzy 



\section{Capítulo}

6

\section{Apêndice}

Neste Apêndice vamos mostrar alguns resultados utilizados na tese.

Lembrando que denotamos $\mathcal{N}=\left\{\left(a_{i}\right)_{i \geq k}, k \in \mathbb{Z} \mid \forall n \geq k,\left(a_{i}\right)_{k \leq i \leq n}\right.$ é uma $R$-representação $\}$, isto é, para todo $n \geq k+3, a_{n} a_{n-1} a_{n-2} a_{n-3}<_{l e x}(a-1)(a-1) 01, a_{k}<_{l e x}(a-1)$, $a_{k+1} a_{k}<_{\text {lex }}(a-1)(a-1)$ e $a_{k+2} a_{k+1} a_{k}<_{l e x}(a-1)(a-1) 0$ e $\mathcal{N}_{f}=\left\{\left(a_{i}\right)_{k \leq i \leq n}, \quad k, n \in\right.$ $\mathbb{Z},\left(a_{i}\right)_{k \leq i \leq n}$ é uma $R$-representação\} e por

$$
\mathcal{D}^{\infty}:=\left\{\left(a_{i}\right)_{i \geq k}, \quad k \in \mathbb{Z}, \forall n \geq k,\left(a_{i}\right)_{k \leq i \leq n} \text { é uma R-representação }\right\}
$$

isto é, $a_{n} a_{n-1} a_{n-2} a_{n-3}<_{\text {lex }}(a-1)(a-1) 01, \forall n \geq k$ onde $a_{k-1}=a_{k-2}=a_{k-3}=0$.

Proposição 6.0.1 Seja $p(x)=x^{3}-a x^{2}+x-1, a \in \mathbb{N}, a \geq 2$ então $P(x)$ possui uma raiz real $\beta>1$ e duas raizes complexas $\alpha, \bar{\alpha}$ tais que $|\alpha|=|\bar{\alpha}|<1$.

Demonstração: Seja $f(x)=a_{3} x^{3}+a_{2} x^{2}+a_{1} x+a_{0}$. O discriminante de $f$ é $\Delta_{f}=a_{2}^{2} a_{1}^{2}-$ $4 a_{3} a_{1}^{3}-4 a_{2}^{3} a_{0}-27 a_{3}^{2} a_{0}^{2}+18 a_{3} a_{2} a_{1} a_{0}$. Sabemos que se $\Delta_{f}<0$ então $f$ tem duas raи́zes complexas conjugadas e uma raiz real. No nosso caso temos que $\Delta_{P}=-4 a^{3}-31+a^{2}+18 a$. É fácil verificar que $\Delta_{P}<0 \forall a \geq 2$, portanto $P(x)$ tem duas raizes complexas conjugadas $\alpha$ e $\bar{\alpha}$ e uma raiz real $\beta$. Por outro lado, temos $P(1)=1-a+1-1=1-a<0$. Como $\lim _{n \longrightarrow+\infty} P(n)=+\infty$, então $\beta>1$. Temos que $\beta \alpha \bar{\alpha}=1$, logo $|\alpha|^{2}=1 / \beta<1$, portanto $|\alpha|<1$.

Lema 6.0.2 i) Todo inteiro natural $n$ pode ser escrito de uma única forma: $n=\sum_{i=2}^{N} \varepsilon_{i} F_{i}$ onde $\left(\varepsilon_{i}\right)_{2 \leq i \leq N} \in \mathcal{N}_{f}$.

ii) Seja $\left(a_{i}\right)_{l \leq i \leq N} \in \mathcal{N}_{f}$ e $\left(b_{i}\right)_{l^{\prime} \leq i \leq \infty} \in \mathcal{N}$ tais que $a_{l}>0$ e $b_{l^{\prime}}>0$. Se $\sum_{i=l}^{N} a_{i} \alpha^{i}=\sum_{i=l^{\prime}}^{\infty} b_{i} \alpha^{i}$ então $l=l^{\prime}$ e para todo $i \geq N, b_{i}=0$ e para todo $l \leq i \leq N, a_{i}=b_{i}$. 
iii) Seja $\left(\varepsilon_{i}\right)_{i \geq 2}$ então $\sum_{i=2}^{\infty} \varepsilon_{i} \alpha^{i} \in \operatorname{int}(\mathcal{R})$. Em particular, $0 \in \operatorname{int}(\mathcal{R})$.

iv) Seja $z \in \mathbb{Z}[\beta] \cap \mathbb{R}^{+}$então existe $\left(a_{i}\right)_{k \leq i \leq l} \in \mathcal{N}_{f}, k \leq l$ tal que $z=\sum_{i=k}^{l} a_{i} \beta^{i}$.

v) Para todo $n \geq 2$ temos que $\beta^{n}=F_{n} \beta^{2}+\left(F_{n-1}-F_{n-2}\right) \beta+F_{n-2}$, em particular se $\left(\varepsilon_{i}\right)_{k \leq i \leq l} \in$ $\mathcal{N}_{f}$ ent $\tilde{a} o \sum_{i=2}^{l} \varepsilon_{i} \beta^{i}=n \beta^{2}+r(n) \beta+s(n)$ onde $n=\sum_{i=2}^{l} \varepsilon_{i} F_{i}, r(n)=\sum_{i=2}^{l} \varepsilon_{i}\left(-F_{i-1}+F_{i-2}\right)$ e $s(n)=\sum_{i=2}^{l} \varepsilon_{i} F_{i-1}$.

vi) Sejam $\left(a_{i}\right)_{l \leq i \leq k}$ e $\left(b_{i}\right)_{l \leq i \leq k}$ pertencentes a $\mathcal{N}_{f}$ então $\sum_{i=l}^{k} a_{i} \beta^{i}<\sum_{i=l}^{k} b_{i} \beta^{i}$ se, e somente se, $\left(a_{i}\right)_{l \leq i \leq k}<_{l e x}\left(b_{i}\right)_{l \leq i \leq k}$.

vii) Sejam $c, d \in \mathbb{R}, \alpha^{2}=c+d \alpha$ então 1, c e d são $\mathbb{Q}$-Linearmente Independentes.

\section{Demonstração:}

i) Como $F_{n+2}=R_{n}$ para todo $n \geq 0$ (ver Capítulo 2), obtemos o resultado usando a Proposição 1.2.1 item (b).

ii) Supomos que $\sum_{i=l}^{N} a_{i} \alpha^{i}=\sum_{i=l^{\prime}}^{+\infty} b_{i} \alpha^{i}$ e que $l=l^{\prime}$. Então temos associado o caminho

$$
\left(a_{l}, b_{l}\right) \ldots\left(a_{N}, b_{N}\right),\left(0, b_{N+1}\right)\left(0, b_{N+2}\right)\left(0, b_{N+3}\right) \ldots
$$

no autômato, o que não ocorre, pois não existe um caminho com esta sequência.

iii) Existe um inteiro $k$ tal que $\alpha^{k} \mathcal{F} \subset \mathcal{R}$. Por outro lado, temos $0 \in \operatorname{int}(\mathcal{F})$ ver [2], [3], logo existe $r>0$, tal que $B(0, r) \subset \mathcal{F}$ e então $B\left(0,\left|\alpha^{k}\right| r\right) \subset \alpha^{k} \mathcal{F} \subset \mathcal{R}$. Portanto, $0 \in \operatorname{int}(\mathcal{R})$.

iv) Seja $z=\sum_{i=0}^{L} c_{i} \beta^{i}, c_{i} \in \mathbb{N}$. Como $\operatorname{Fin}(\beta)=\mathbb{Z}[1 / \beta] \cap \mathbb{R}^{+}$existe $\left(a_{i}\right)_{k \leq i \leq l} \in \mathcal{N}_{f}$ tal que $\sum_{i=0}^{L} c_{i} \beta^{i}=\sum_{i=k}^{l} a_{i} \beta^{i}$. Seja s um inteiro natural tal que $s+k \geq 0$, onde $\beta$ é uma raiz do polinômio $Q(X)=X^{s} \times\left(\sum_{i=0} c_{i} X^{i}-\sum_{i=k}^{l} a_{i} X^{i}\right)$. Como $\alpha$ e $\beta$ são conjugados, $Q(X)$ é um polinômio múltiplo do polinômio minimal de $\alpha$. Portanto, $z=\sum_{i=0}^{L} c_{i} \alpha^{i}=\sum_{i=k}^{l} a_{i} \alpha^{i}$.

v) A prova, deixada ao leitor, pode ser feita por indução.

vi) Ver Capítulo 1.

vii) Sejam $c, d \in \mathbb{R}$ tais que $\alpha^{2}=c+\alpha d$.

Aplicando o algoritmo da divisão para os polinômios $P(x)=x^{3}-a x^{2}+x-1$, e $Q(x)=$ $x^{2}-c x-d$, podemos escrever $P(x)=N(x) Q(x)+R(x)$ onde $N(x)$ é um polinômio de grau 1 e $R(x)=[c(c-a)+(d-b)] \alpha+(d(c-a)-1)$. Note que $R(\alpha)=0$. 
Como $\alpha \in \mathbb{C} \backslash \mathbb{R}$ segue que

$$
\left\{\begin{array}{cc}
c(c-a)+(d-b) & =0 \\
d(c-a)-1 & =0
\end{array}\right.
$$

Supohamos que $1, c, d$ são linearmente dependentes, isto é, existem $p, q, r \in \mathbb{Z}$ não todos nulos tais que:

$$
p+q \cdot c+r \cdot d=0
$$

Multiplicando a primeira equação de (6.1) por q, a segunda por $r$ e somando ambas obtemos

$$
-p \cdot c+q \cdot d+(p \cdot a-q \cdot b)=r
$$

e dai temos o sistema:

$$
\left\{\begin{array}{ccc}
-p \cdot c+q \cdot d & = & r-p \cdot a+q \cdot b \\
q \cdot c+r \cdot d & = & -p .
\end{array}\right.
$$

Caso 1.1 Se $-p r-q^{2} \neq 0$. Neste caso a solução é

$$
c=\frac{r^{2}-r \cdot p \cdot a+r \cdot q \cdot b+q \cdot p}{-r \cdot p-q^{2}} \in \mathbb{Q} .
$$

Logo $d \in \mathbb{Q}$, logo $\alpha^{2}=c \alpha+d=\frac{s}{t} \alpha+\frac{m}{n}$. Seja $p=m m c(t, m)$, logo existe $k, l \in \mathbb{Z}$ tais que $p \alpha^{2}=k \alpha+t$, assim $\alpha$ é um inteiro algébrico de grau 2 o que é uma contradição.

Caso 1.2 Se $q^{2}+p \cdot r=0$, consideremos o sistema

$$
\left\{\begin{array}{c}
c \cdot \alpha+d=\alpha^{2} \\
c \cdot q+d \cdot r=-p
\end{array}\right.
$$

Vamos considerar $\alpha \cdot r-q \neq 0 \Longrightarrow q \neq \alpha \cdot r$.

Daí,

$$
c=\frac{r \alpha^{2}+p}{\alpha r-q}
$$

$e$

$$
d=\frac{-\alpha p-\alpha^{2} q}{\alpha r-q}
$$

Caso 1.2.2 Se $r \neq 0$, temos $p=\frac{-q^{2}}{r}$. Assim $c=\frac{r \alpha^{2}+p}{\alpha r-q}=\frac{r \alpha^{2}-\frac{q^{2}}{r}}{r \alpha-q}=\frac{r \alpha-q}{r}=\alpha-\frac{q}{r}$. Logo, $c=\alpha-\frac{q}{r} \Longrightarrow \alpha=c+\frac{q}{r} \in \mathbb{R}$, o que é uma contradição!

Portanto, $r=0$ e $q=0$ o que implica que $p=0$.

Teorema 6.0.3 : A correspondência $f$ é uma função continua, bijetora e satisfaz $f(0)=u$ 
$e f(1)=w$.

\section{Demonstração da injetividade e continuidade}

fé injetora: Vamos considerar $a_{i}=a_{i}^{\prime}, 1 \leq i \leq k-1$.

Temos que $f(t)=f\left(t^{\prime}\right) \Longleftrightarrow g_{b_{k}}(z)=g_{b_{k}^{\prime}}\left(z^{\prime}\right) \Longleftrightarrow\left(b_{k}=0, z=-1-\alpha^{3}=u, b_{k}^{\prime}=1, z^{\prime}=\right.$ $\left.\alpha^{2}-\alpha^{3}=w\right)$ ou $\left(b_{k}=1, b_{k}^{\prime}=2, z=z^{\prime}=-1-\alpha^{3}=u\right)$.

Caso 1 Se $b_{k}=0, z=-1-\alpha^{3}=u, b_{k}^{\prime}=1, z^{\prime}=\alpha^{2}-\alpha^{3}=w$ e como as representaçôes de $-1-\alpha^{3}=u$ e $\alpha^{2}-\alpha^{3}=w$ são $(0 \overline{2})$ e $(\overline{2})$ respectivamente, então as sequências $\left(b_{i}\right)$ e $\left(b_{i}^{\prime}\right)$ são dadas por: $\left(b_{i}\right)=b_{1} b_{2} \ldots b_{k-1} 00 \overline{2} e\left(b_{i}^{\prime}\right)=b_{1} b_{2} \ldots b_{k-1} 1 \overline{2}$.

Se $b_{k}^{\prime}=1$ então pela equação (4.15) $a_{k}^{\prime}=1$ e como $b_{k}=0$ temos que considerar os seguintes casos:

Caso 1.1: Se $a_{k-1}=2$ então pela equação (4.16) $a_{k}=0$ e as sequências $\left(a_{i}\right)_{i \geq 1}$ e $\left(a_{i}^{\prime}\right)_{i \geq 1}$ são das seguintes formas:

$\left(a_{i}\right)_{i \geq 1}: a_{1} a_{2} \ldots a_{k-2} 20 a_{k+1} \ldots$ e $\left(a_{i}^{\prime}\right)_{i \geq 1}: a_{1} a_{2} \ldots a_{k-2} 21 a_{k+1}^{\prime} \ldots \ldots$

Sendo $\psi\left(a_{i}\right)=\left(b_{i}\right)$ e $\psi\left(a_{i}^{\prime}\right)=\left(b_{i}^{\prime}\right)$, como $b_{i}=2$ para todo $i \geq k+1$, pela equação (4.16) temos $a_{i}=2, \forall i \geq k+1$. Como $b_{j}^{\prime}=2$ para todo $j \geq k+1$ então pela equação (4.17)(a) $a_{j}^{\prime}=0, \forall j \geq k+1$. Assim, $\left(a_{i}\right)_{i \geq 1}=a_{1} a_{2} \ldots a_{k-2} 20 \overline{2} e\left(a_{i}^{\prime}\right)_{i \geq 1}=a_{1} a_{2} \ldots a_{k-2} 21 \overline{0}$. Logo, pelo Lema 4.1.2, $t=t^{\prime}$.

Caso 1.2: Se $a_{k-1}=0$ e $b_{k}=0$ então usando a equação (4.17)(b) $a_{k}=2$ e as sequências $\left(a_{i}\right)_{i \geq 1}$ e $\left(a_{i}^{\prime}\right)_{i \geq 1}$ são das seguintes formas:

$\left(a_{i}\right)=a_{1} a_{2} \ldots a_{k-2} 02 a_{k+1} \ldots$ e $\left(a_{i}^{\prime}\right)=a_{1} a_{2} \ldots a_{k-2} 01 a_{k+1}^{\prime} \ldots \ldots$

Se $\psi\left(a_{i}\right)=\left(b_{i}\right)$ e $\psi\left(a_{i}^{\prime}\right)=\left(b_{i}^{\prime}\right)$, como $a_{k-1}=0$ e $a_{k}=2$ então pela equação (4.17) (b) temos $b_{k}=0$ e como $b_{k+1}=0$ então pela equação $(4.17)(b)$ temos $a_{k+1}=2$. Como $b_{i}=2, \forall i \geq k+2$ pela equação (4.17)(a) temos que $a_{i}=0$ para todo $i \geq k+2$. Agora, como $b_{j}^{\prime}=2, \forall j \geq k+1$ temos pela equação (4.16) que $a_{j}^{\prime}=2, \forall j \geq k+1$. Assim, $\left(a_{i}\right)_{i \geq 1}=a_{1} a_{2} \ldots a_{k-2} 02 \overline{0} e$ $\left(a_{i}^{\prime}\right)_{i \geq 1}=a_{1} a_{2} \ldots a_{k-2} 01 \overline{2}$. Logo, pelo Lema 4.1.2, $t=t^{\prime}$.

Caso 1.3: Se $a_{r-1}=0, a_{r}=\ldots=a_{k-1}=1$ e \# $(k-r)$ é par e como $b_{k}=0$ então pela equação (4.19) $a_{k}=2$ e as sequências $\left(a_{i}\right)_{i \geq 1}$ e $\left(a_{i}^{\prime}\right)_{i \geq 1}$ são das seguintes formas: $\left(a_{i}\right)_{i \geq 1}=a_{1} \ldots a_{r-2} 011 \ldots 12 a_{k+1} \ldots e$ e $\left(a_{i}^{\prime}\right)_{i \geq 1}=a_{1} \ldots a_{r-2} 011 \ldots 11 a_{k+1}^{\prime} \ldots$.

Como $\psi\left(a_{i}\right)=\left(b_{i}\right)$ e $\psi\left(a_{i}^{\prime}\right)=\left(b_{i}^{\prime}\right)$, usando a equação (4.17)(a) para determinar os elementos $a_{i}$ e a equação (4.16) para os elementos $a_{i}^{\prime}$ temos $\left(a_{i}\right)_{i \geq 1}=a_{1} \ldots a_{r-2} 011 \ldots 12 \overline{0} e$ $\left(a_{i}^{\prime}\right)_{i \geq 1}=a_{1} \ldots a_{r-2} 011 \ldots 11 \overline{2}$. Assim, pelo Lema 4.1.2, $t=t^{\prime}$.

Caso 1.4: Se $a_{r-1}=0, a_{r}=\ldots=a_{k-1}=1 e \#(k-r)$ é ímpar e como $b_{k}=0$ então pela equação (4.19)(a) $a_{k}=0$ e assim sequências $\left(a_{i}\right)_{i \geq 1}$ e $\left(a_{i}^{\prime}\right)_{i \geq 1}$ são das seguintes formas: 
$\left(a_{i}\right)=a_{1} \ldots a_{r-2} 011 \ldots 10 a_{k+1} \ldots e\left(a_{i}^{\prime}\right)=a_{1} \ldots a_{r-2} 011 \ldots 11 a_{k+1}^{\prime} \ldots$.

Como $\psi\left(a_{i}\right)=\left(b_{i}\right)$ e $\psi\left(a_{i}^{\prime}\right)=\left(b_{i}^{\prime}\right)$, pelas equaçãos (4.17)(a) e (4.16) para determinar os elementos $a_{i}$ e pelas equações (4.19)(a) e (4.16) para os elementos $a_{i}^{\prime}$ obtemos $\left(a_{i}\right)_{i \geq 1}=a_{1} \ldots a_{r-2} 011 \ldots 10 \overline{2} e\left(a_{i}^{\prime}\right)_{i \geq 1}=a_{1} \ldots a_{r-2} 011 \ldots 11 \overline{2}$. Logo, pelo Lema 4.1.2, $t=t^{\prime}$.

Caso 1.5: Se $a_{r-1}=2, a_{r}=\ldots=a_{k-1}=1, \#(k-r)$ é par e como $b_{k}=0$ então pela equação (4.18)(a) $a_{k}=0$ e assim as sequências $\left(a_{i}\right)_{i \geq 1}$ e $\left(a_{i}^{\prime}\right)_{i \geq 1}$ são das seguintes formas: $\left(a_{i}\right)_{i \geq 1}=a_{1} \ldots a_{r-2} 211 \ldots 10 a_{k+1} \ldots e\left(a_{i}^{\prime}\right)_{i \geq 1}=a_{1} \ldots a_{r-2} 211 \ldots 11 a_{k+1}^{\prime} \ldots \ldots$

Como $\psi\left(a_{i}\right)=\left(b_{i}\right)$ e $\psi\left(a_{i}^{\prime}\right)=\left(b_{i}^{\prime}\right)$, pelas equaçôes (4.17)(b) e (4.16) para determinar os elementos $a_{i}$ e pelas equações (4.18)(b) e (4.16) para os elementos $a_{i}^{\prime}$ temos que $\left(a_{i}\right)_{i \geq 1}=$ $a_{1} \ldots a_{r-2} 211 \ldots 10 \overline{2}$ e $\left(a_{i}^{\prime}\right)_{i \geq 1}=a_{1} \ldots a_{r-2} 211 \ldots 11 \overline{0}$. Logo, pelo Lema 4.1.2, $t=t^{\prime}$.

Caso 1.6: Se $a_{r-1}=2, a_{r}=\ldots=a_{k-1}=1$ e \#(k-r) ímpar e como $b_{k}=0$ então pela equação (4.18)(b) $a_{k}=2$ e as sequências $\left(a_{i}\right)_{i \geq 1}$ e $\left(a_{i}^{\prime}\right)_{i \geq 1}$ são das seguintes formas: $\left(a_{i}\right)_{i \geq 1}=a_{1} \ldots a_{r-2} 211 \ldots 12 a_{k+1} \ldots$ e $\left(a_{i}^{\prime}\right)_{i \geq 1}=a_{1} \ldots a_{r-2} 211 \ldots 11 a_{k+1}^{\prime} \ldots$.

Como $\psi\left(a_{i}\right)=\left(b_{i}\right)$ e $\psi\left(a_{i}^{\prime}\right)=\left(b_{i}^{\prime}\right)$, pelas equaçôes (4.18)(b) e (4.17)(a) para determinar $a_{i} e$ as equaçôes (4.18)(a) e (4.16) para os elementos $a_{i}^{\prime}$ temos que $\left(a_{i}\right)_{i \geq 1}=a_{1} \ldots a_{r-2} 211 \ldots 12 \overline{0} e$ $\left(a_{i}^{\prime}\right)_{i \geq 1}=a_{1} \ldots a_{r-2} 211 \ldots 11 \overline{2}$. Assim, pelo Lema 4.1.2, $t=t^{\prime}$.

Caso 1.7: Se $a_{1}=a_{2}=\ldots=a_{k-1}=1$ e \# $(k-1)$ é par e como $b_{k}=0$ então pela equação (4.18)(a) $a_{k}=0$ e as sequências $\left(a_{i}\right)_{i \geq 1}$ e $\left(a_{i}^{\prime}\right)_{i \geq 1}$ são das seguintes formas: $\left(a_{i}\right)_{i \geq 1}=$ $111 \ldots 10 a_{k+1} \ldots$ e $\left(a_{i}^{\prime}\right)_{i \geq 1}=111 \ldots 11 a_{k+1}^{\prime} \ldots$.

Como $\psi\left(a_{i}\right)=\left(b_{i}\right)$ e $\psi\left(a_{i}^{\prime}\right)=\left(b_{i}^{\prime}\right)$, pelas equações (4.18)(a) e (4.17)(b) para determinar os elementos $a_{i}$ e as equaçôes (4.18)(b) e (4.17)(a) para os elementos $a_{i}^{\prime}$ temos que $\left(a_{i}\right)_{i \geq 1}=$ $111 \ldots 10 \overline{2}$ e $\left(a_{i}^{\prime}\right)_{i \geq 1}=111 \ldots 11 \overline{0}$. Assim, pelo Lema 4.1.2, $t=t^{\prime}$.

Caso 1.8: Se $a_{1}=a_{2}=\ldots=a_{k-1}=1$ e \# $(k-1)$ é impar e como $b_{k}=0$ então pela equação (4.18)(b) $a_{k}=2$ e as sequências $\left(a_{i}\right)_{i \geq 1} e\left(a_{i}^{\prime}\right)_{i \geq 1}$ são das seguintes formas: $\left(a_{i}\right)_{i \geq 1}=111 \ldots 12 a_{k+1} \ldots e\left(a_{i}^{\prime}\right)_{i \geq 1}=111 \ldots 11 a_{k+1}^{\prime} \ldots$.

Como $\psi\left(a_{i}\right)=\left(b_{i}\right)$ e $\psi\left(a_{i}^{\prime}\right)=\left(b_{i}^{\prime}\right)$, pelas equações (4.17)(b) e (a) para determinar os elementos $a_{i}$ e as equações (4.18) e (4.16) para os elementos $a_{i}^{\prime}$ temos que $\left(a_{i}\right)_{i \geq 1}=111 \ldots 12 \overline{0} e$ $\left(a_{i}^{\prime}\right)_{i \geq 1}=111 \ldots 11 \overline{2}$. Portanto, pelo Lema 4.1.2, $t=t^{\prime}$.

Caso 2 Se $\left(b_{k}=1, b_{k}^{\prime}=2, z=z^{\prime}=-1-\alpha^{3}=u\right)$.

Neste caso, como a representação de $-1-\alpha^{3}=u$ é $(0 \overline{2})$, então as sequências $\left(b_{i}\right)$ e $\left(b_{i}^{\prime}\right)$ são dadas por: $\left(b_{i}\right): b_{1} b_{2} \ldots b_{k-1} 10 \overline{2}$ e $\left(b_{i}^{\prime}\right): b_{1} b_{2} \ldots b_{k-1} 20 \overline{2}$.

Se $b_{k}=1$ então pela equação (4.15) $a_{k}=1$ e como $b_{k+1}^{\prime}=0$ temos que considerar os seguintes casos: 
Caso 2.1: Se $a_{k-1}=2$ e $b_{k}^{\prime}=2$ então pela equação (4.16) temos $a_{k}^{\prime}=2$ e as sequências $\left(a_{i}\right)_{i \geq 1}$ e $\left(a_{i}^{\prime}\right)_{i \geq 1}$ são das seguintes formas:

$\left(a_{i}\right)_{i \geq 1}: a_{1} a_{2} \ldots a_{k-2} 21 a_{k+1} \ldots$ e $\left(a_{i}^{\prime}\right)_{i \geq 1}: a_{1} a_{2} \ldots a_{k-2} 22 a_{k+1}^{\prime} \ldots$.

Como $\psi\left(a_{i}\right)=\left(b_{i}\right)$ e $\psi\left(a_{i}^{\prime}\right)=\left(b_{i}^{\prime}\right)$, então usando a equação (4.16) para determinar os elementos $a_{i}$ e as equaçôes (4.17)(b) e (4.16) para os elementos de $a_{i}^{\prime}$ temos

$\left(a_{i}\right)_{i \geq 1}: a_{1} a_{2} \ldots a_{k-2} 21 \overline{2}$ e $\left(a_{i}^{\prime}\right)_{i \geq 1}: a_{1} a_{2} \ldots a_{k-2} 22 \overline{0}$. Assim, pelo Lema 4.1.2, $t=t^{\prime}$.

Caso 2.2: Se $a_{k-1}=0$ e $b_{k}^{\prime}=2$ então pela equação (4.17)(b) $a_{k}^{\prime}=0$ e as sequências $\left(a_{i}\right)_{i \geq 1}$ $e\left(a_{i}^{\prime}\right)_{i \geq 1}$ são das seguintes formas:

$\left(a_{i}\right)_{i \geq 1}: a_{1} a_{2} \ldots a_{k-2} 01 a_{k+1} \ldots$ e $\left(a_{i}^{\prime}\right)_{i \geq 1}: a_{1} a_{2} \ldots a_{k-2} 00 a_{k+1}^{\prime} \ldots$.

Como $\psi\left(a_{i}\right)=\left(b_{i}\right)$ e $\psi\left(a_{i}^{\prime}\right)=\left(b_{i}^{\prime}\right)$, então pela equação (4.17)(a) para determinar os elementos $a_{i}$ e as equaçôes (4.17)(a) e (4.16) para os elementos $a_{i}^{\prime}$ temos

$\left(a_{i}\right)_{i \geq 1}: a_{1} a_{2} \ldots a_{k-2} 01 \overline{0}$ e $\left(a_{i}^{\prime}\right)_{i \geq 1}: a_{1} a_{2} \ldots a_{k-2} 00 \overline{2}$. Assim, pelo Lema 4.1.2., $t=t^{\prime}$.

Caso 2.3: Se $a_{r-1}=0, a_{r}=\ldots=a_{k-1}=1$ e \# $(k-r)$ é par e como $b_{k}^{\prime}=2$ então pela equação (4.19)(a) temos $a_{k}^{\prime}=0$ e as sequências $\left(a_{i}\right)_{i \geq 1} e\left(a_{i}^{\prime}\right)_{i \geq 1}$ são das seguintes formas:

$\left(a_{i}\right)_{i \geq 1}: a_{1} \ldots a_{r-2} 011 \ldots 11 a_{k+1} \ldots$ e $\left(a_{i}^{\prime}\right)_{i \geq 1}: a_{1} \ldots a_{r-2} 011 \ldots 10 a_{k+1}^{\prime} \ldots$.

Como $\psi\left(a_{i}\right)=\left(b_{i}\right)$ e $\psi\left(a_{i}^{\prime}\right)=\left(b_{i}^{\prime}\right)$, então pelas equações (4.19)(a) e (4.17)(a) para determinar os elementos $a_{i}$ e (4.19)(b), (4.17)(b) e (4.16) para os elementos $a_{i}^{\prime}$ temos

$\left(a_{i}\right)_{i \geq 1}: a_{1} \ldots a_{r-2} 011 \ldots 11 \overline{0}$ e $\left(a_{i}^{\prime}\right)_{i \geq 1}: a_{1} \ldots a_{r-2} 011 \ldots 10 \overline{2}$. Logo, pelo Lema 4.1.2, $t=t^{\prime}$.

Caso 2.4: Se $a_{r-1}=0, a_{r}=\ldots=a_{k-1}=1$ e\# $(k-r)$ é ímpar e como $b_{k}^{\prime}=2$ então pela equação (4.19)(b) $a_{k}^{\prime}=2$ e as sequências $\left(a_{i}\right)_{i \geq 1}$ e $\left(a_{i}^{\prime}\right)_{i \geq 1}$ são das seguintes formas:

$\left(a_{i}\right)_{i \geq 1}: a_{1} \ldots a_{r-2} 011 \ldots 11 a_{k+1} \ldots$ e $\left(a_{i}^{\prime}\right)_{i \geq 1}: a_{1} \ldots a_{r-2} 011 \ldots 12 a_{k+1}^{\prime} \ldots$.

Como $\psi\left(a_{i}\right)=\left(b_{i}\right)$ e $\psi\left(a_{i}^{\prime}\right)=\left(b_{i}^{\prime}\right)$, então pelas equações (4.19)(b) e (4.16) para determinar os elementos $a_{i}$ e as equaçôes $(4.19)(a),(4.17)(b)$ e (a) para os elementos $a_{i}^{\prime}$ temos $\left(a_{i}\right)_{i \geq 1}: a_{1} \ldots a_{r-2} 011 \ldots 11 \overline{2}$ e $\left(a_{i}^{\prime}\right)_{i \geq 1}: a_{1} \ldots a_{r-2} 011 \ldots 12 \overline{0}$. Logo, pelo Lema 4.1.2, $t=t^{\prime}$.

Caso 2.5: Se $a_{r-1}=2, a_{r}=\ldots=a_{k-1}=1$ e \# $(k-r)$ é par e como $b_{k}^{\prime}=2$ então pela equação (4.18)(a) $a_{k}^{\prime}=2$ e as sequências $\left(a_{i}\right)_{i \geq 1}$ e $\left(a_{i}^{\prime}\right)_{i \geq 1}$ são das seguintes formas:

$\left(a_{i}\right)_{i \geq 1}: a_{1} \ldots a_{r-2} 211 \ldots 11 a_{k+1} \ldots$ e $\left(a_{i}^{\prime}\right)_{i \geq 1}: a_{1} \ldots a_{r-2} 211 \ldots 12 a_{k+1}^{\prime} \ldots$.

Como $\psi\left(a_{i}\right)=\left(b_{i}\right)$ e $\psi\left(a_{i}^{\prime}\right)=\left(b_{i}^{\prime}\right)$, então pelas equaçôes (4.18)(b) e (4.16) para determinar os elementos $a_{i}$ e (4.18)(a) e (4.17)(a) para os elementos $a_{i}^{\prime}$ temos

$\left(a_{i}\right)_{i \geq 1}: a_{1} \ldots a_{r-2} 211 \ldots 11 \overline{2}$ e $\left(a_{i}^{\prime}\right)_{i \geq 1}: a_{1} \ldots a_{r-2} 211 \ldots 12 \overline{0}$.

Assim, pelo Lema 4.1.2, $t=t^{\prime}$.

Caso 2.6: Se $a_{r-1}=2, a_{r}=\ldots=a_{k-1}=1$ e\# $(k-r)$ é impar e como $b_{k}^{\prime}=2$ então pela equação (4.18)(b) $a_{k}^{\prime}=0$ e as sequências $\left(a_{i}\right)_{i \geq 1}$ e $\left(a_{i}^{\prime}\right)_{i \geq 1}$ são das seguintes formas:

$\left(a_{i}\right)_{i \geq 1}: a_{1} \ldots a_{r-2} 211 \ldots 11 a_{k+1} \ldots$ e $\left(a_{i}^{\prime}\right)_{i \geq 1}: a_{1} \ldots a_{r-2} 211 \ldots 10 a_{k+1}^{\prime} \ldots$. 
Como $\psi\left(a_{i}\right)=\left(b_{i}\right)$ e $\psi\left(a_{i}^{\prime}\right)=\left(b_{i}^{\prime}\right)$, então pelas equações (4.18)(a) e (4.17)(a) para os elementos $a_{i}$ e as equações (4.18)(b), (4.17)(b) e (4.16) para os elementos $a_{i}^{\prime}$ temos $\left(a_{i}\right)_{i \geq 1}$ : $a_{1} \ldots a_{r-2} 211 \ldots 11 \overline{0}$ e $\left(a_{i}^{\prime}\right)_{i \geq 1}: a_{1} \ldots a_{r-2} 211 \ldots 10 \overline{2}$. Logo, pelo Lema 4.1.2, $t=t^{\prime}$.

Caso 2.7: Se $a_{1}=a_{2}=\ldots=a_{k-1}=1$ e \# $(k-1)$ é par e como $b_{k}^{\prime}=2$ então pela equação (4.18)(a) $a_{k}^{\prime}=2$ e as sequências $\left(a_{i}\right)_{i \geq 1} e\left(a_{i}^{\prime}\right)_{i \geq 1}$ são das seguintes formas:

$\left(a_{i}\right)_{i \geq 1}: 111 \ldots 11 a_{k+1} \ldots$ e $\left(a_{i}^{\prime}\right)_{i \geq 1}: 111 \ldots 12 a_{k+1}^{\prime} \ldots$.

Como $\psi\left(a_{i}\right)=\left(b_{i}\right)$ e $\psi\left(a_{i}^{\prime}\right)=\left(b_{i}^{\prime}\right)$, então pelas equações (4.18)(b) e (4.16) para os elementos $a_{i}$ e as equaçôes (4.18)(a), (4.17)(b) e (a) para os elementos $a_{i}^{\prime}$ temos $\left(a_{i}\right)_{i \geq 1}: 111 \ldots 11 \overline{2} e$ $\left(a_{i}^{\prime}\right)_{i \geq 1}: 111 \ldots 12 \overline{0}$. Assim, pelo Lema 4.1.2, $t=t^{\prime}$.

Caso 2.8: Se $a_{1}=a_{2}=\ldots=a_{k-1}=1$ e \# $(k-1)$ é ímpar e como $b_{k}^{\prime}=2$ então pela equação (4.18)(b) $a_{k}^{\prime}=0$ e as sequências $\left(a_{i}\right)_{i \geq 1} e\left(a_{i}^{\prime}\right)_{i \geq 1}$ são das seguintes formas:

$\left(a_{i}\right)_{i \geq 1}: 111 \ldots 11 a_{k+1} \ldots$ e $\left(a_{i}^{\prime}\right)_{i \geq 1}: 111 \ldots 10 a_{k+1}^{\prime} \ldots$.

Como $\psi\left(a_{i}\right)=\left(b_{i}\right)$ e $\psi\left(a_{i}^{\prime}\right)=\left(b_{i}^{\prime}\right)$, então pelas equaçôes (4.18)(a) e (4.17) para determinar os elementos $a_{i}$ e as equaçôes (4.18)(b) e (4.16) para os elementos $a_{i}^{\prime}$ temos $\left(a_{i}\right)_{i \geq 1}: 111 \ldots 11 \overline{0} e$ $\left(a_{i}^{\prime}\right)_{i \geq 1}: 111 \ldots 10 \overline{2}$. Portano, pelo Lema 4.1.2, $t=t^{\prime}$.

fé contínua: Sejam $t=\sum_{i=1}^{\infty} a_{i} 3^{-i}$ e $t^{\prime}=\sum_{i=1}^{\infty} a_{i}^{\prime} 3^{-i}$. Suponhamos que $0<\left|t-t^{\prime}\right|<$ $3^{-N}, N \in \mathbb{N}$ e $N>k$, pelo Lema4.1.2 temos que $a_{k}^{\prime}=a_{k}+1, a_{i}^{\prime}=0$ e $a_{i}=2$ para todo $i$ que satisfaz $k+1 \leq i \leq N$. Temos que as seguintes sequências:

$$
\begin{aligned}
t & =\left(a_{1} \ldots a_{k-1} a_{k} 222 \ldots 2\right) \\
t^{\prime} & =\left(a_{1} \ldots a_{k-1} a_{k+1} 000 \ldots 0\right) .
\end{aligned}
$$

Vamos analisar os casos para $X=\mathcal{R}_{\alpha-1}$.

Caso 1.1: Se $a_{k-1}=0, a_{k}=0, a_{k}^{\prime}=1$ então temos as sequências:

$$
\begin{aligned}
t & =\left(a_{1} \ldots a_{k-2} 00222 \ldots 2\right) \\
t^{\prime} & =\left(a_{1} \ldots a_{k-2} 01000 \ldots 0\right) .
\end{aligned}
$$

Assim,

$$
\left|f(t)-f\left(t^{\prime}\right)\right|=\left|g_{2} g_{0} g_{2}^{N-k-1}\left(y_{1}\right)-g_{1} g_{0} g_{2}^{N-k-1}\left(y_{1}^{\prime}\right)\right| \times|\alpha|^{2(k-1)} .
$$

Sejam $z_{1}=g_{0} g_{2}^{N-k-1}\left(y_{1}\right)$ e $z_{1}^{\prime}=g_{0} g_{2}^{N-k-1}\left(y_{1}^{\prime}\right)$, dai

$$
\left|f(t)-f\left(t^{\prime}\right)\right|=\left|g_{2}\left(z_{1}\right)-g_{1}\left(z_{1}^{\prime}\right)\right| \times|\alpha|^{2(k-1)}
$$


Como $g_{2}\left(-1-\alpha^{3}\right)=g_{1}\left(-1-\alpha^{3}\right)=-\frac{1}{\alpha}-\alpha-\alpha^{4}-\alpha^{6}$ temos

$$
\begin{array}{rlrl}
\left|f(t)-f\left(t^{\prime}\right)\right| & =\left|g_{2}\left(z_{1}\right)-g_{2}\left(-1-\alpha^{3}\right)+g_{1}\left(-1-\alpha^{3}\right)-g_{1}\left(z_{1}^{\prime}\right)\right| \times|\alpha|^{2(k-1)} \\
& \leq\left|g_{2}\left(z_{1}\right)-g_{2}\left(-1-\alpha^{3}\right)\right|+\left|g_{1}\left(-1-\alpha^{3}\right)-g_{1}\left(z_{1}^{\prime}\right)\right| \times|\alpha|^{2(k-1)} \\
& \leq & \left(|\alpha|^{4}+|\alpha|^{3}\right)|\alpha|^{2(k-1)} \times \operatorname{diam}(X) \\
& \leq & (1+|\alpha|)|\alpha|^{2 k+1} \times \operatorname{diam}(X) .
\end{array}
$$

Caso 1.2: Se $a_{k-1}=2, a_{k}=0, a_{k}^{\prime}=1$ então temos as sequências:

$$
\begin{aligned}
t & =\left(a_{1} \ldots a_{k-2} 20222 \ldots 2\right) \\
t^{\prime} & =\left(a_{1} \ldots a_{k-2} 21000 \ldots 0\right) .
\end{aligned}
$$

Assim,

$$
\left|f(t)-f\left(t^{\prime}\right)\right|=\left|g_{2} g_{0} g_{2}^{N-k-1}\left(y_{2}\right)-g_{1} g_{0} g_{2}^{N-k-1}\left(y_{2}^{\prime}\right)\right| \times|\alpha|^{2(k-1)} \text {. }
$$

Sejam $z_{2}=g_{0} g_{2}^{N-k-1}\left(y_{2}\right)$ e $z_{2}^{\prime}=g_{0} g_{2}^{N-k-1}\left(y_{2}^{\prime}\right)$, dai

$$
\left|f(t)-f\left(t^{\prime}\right)\right|=\left|g_{2}\left(z_{2}\right)-g_{1}\left(z_{2}^{\prime}\right)\right| \times|\alpha|^{2(k-1)}
$$

Usando o mesmo argumento temos:

$$
\begin{array}{rlrl}
\left|f(t)-f\left(t^{\prime}\right)\right| & =\left|g_{2}\left(z_{2}\right)-g_{2}\left(-1-\alpha^{3}\right)+g_{1}\left(-1-\alpha^{3}\right)-g_{1}\left(z_{2}^{\prime}\right)\right| \times|\alpha|^{2(k-1)} \\
& \leq\left|g_{2}\left(z_{2}\right)-g_{2}\left(-1-\alpha^{3}\right)\right|+\left|g_{1}\left(-1-\alpha^{3}\right)-g_{1}\left(z_{2}^{\prime}\right)\right| \times|\alpha|^{2(k-1)} \\
& \leq & \left(|\alpha|^{4}+|\alpha|^{3}\right)|\alpha|^{2(k-1)} \times \operatorname{diam}(X) \\
& \leq & (1+|\alpha|)|\alpha|^{2 k+1} \times \operatorname{diam}(X)
\end{array}
$$

Caso 1.3: Se $a_{i-1}=0, a_{i}=\ldots=a_{k-1}=1, a_{k}=0, a_{k}^{\prime}=1$ e \# $(k-i-1)$ é par então temos as sequências:

$$
\begin{aligned}
t & =\left(a_{1} \ldots 01 \ldots 102222 \ldots\right) \\
t^{\prime} & =\left(a_{1} \ldots 01 \ldots 1100000 \ldots\right)
\end{aligned}
$$

Assim,

$$
\left|f(t)-f\left(t^{\prime}\right)\right|=\left|g_{2} g_{0} g_{2}^{N-k-1}\left(y_{3}\right)-g_{1} g_{0} g_{2}^{N-k-1}\left(y_{3}^{\prime}\right)\right| \times|\alpha|^{2(k-1)} .
$$

Sejam $z_{3}=g_{0} g_{2}^{N-k-1}\left(y_{3}\right)$ e $z_{3}^{\prime}=g_{0} g_{2}^{N-k-1}\left(y_{3}^{\prime}\right)$, daí

$$
\left|f(t)-f\left(t^{\prime}\right)\right|=\left|g_{2}\left(z_{3}\right)-g_{1}\left(z_{3}^{\prime}\right)\right| \times|\alpha|^{2(k-1)}
$$


Usando o mesmo argumento temos:

$$
\begin{array}{rlrl}
\left|f(t)-f\left(t^{\prime}\right)\right| & =\left|g_{2}\left(z_{3}\right)-g_{2}\left(-1-\alpha^{3}\right)+g_{1}\left(-1-\alpha^{3}\right)-g_{1}\left(z_{3}^{\prime}\right)\right| \times|\alpha|^{2(k-1)} \\
& \leq\left|g_{2}\left(z_{3}\right)-g_{2}\left(-1-\alpha^{3}\right)\right|+\left|g_{1}\left(-1-\alpha^{3}\right)-g_{1}\left(z_{3}^{\prime}\right)\right| \times|\alpha|^{2(k-1)} \\
& \leq & \left(|\alpha|^{4}+|\alpha|^{3}\right) \times|\alpha|^{2 k-1} \times \operatorname{diam}(X) \\
& \leq & (1+|\alpha|) \times|\alpha|^{2 k+1} \times \operatorname{diam}(X)
\end{array}
$$

Caso 1.4: Se $a_{i-1}=0, a_{i}=\ldots=a_{k-1}=1, a_{k}=0, a_{k}^{\prime}=1$ e \#(k-i-1) é impar então temos as sequências:

$$
\begin{aligned}
t & =\left(a_{1} \ldots 01 \ldots 102222 \ldots\right) \\
t^{\prime} & =\left(a_{1} \ldots 01 \ldots 1100000 \ldots\right)
\end{aligned}
$$

Assim,

$$
\left|f(t)-f\left(t^{\prime}\right)\right|=\left|g_{1} g_{0} g_{2}^{N-k-1}\left(y_{4}\right)-g_{2} g_{0} g_{2}^{N-k-1}\left(y_{4}^{\prime}\right)\right| \times|\alpha|^{2(k-1)} .
$$

Considere $z_{4}=g_{0} g_{2}^{N-k-1}\left(y_{4}\right)$ e $z_{4}^{\prime}=g_{0} g_{2}^{N-k-1}\left(y_{4}^{\prime}\right)$, dai

$$
\left|f(t)-f\left(t^{\prime}\right)\right|=\left|g_{1}\left(z_{4}\right)-g_{2}\left(z_{4}^{\prime}\right)\right| \times|\alpha|^{2(k-1)}
$$

Usando o mesmo argumento temos:

$$
\begin{array}{rlrl}
\left|f(t)-f\left(t^{\prime}\right)\right| & = & \left|g_{1}\left(z_{4}\right)-g_{1}\left(-1-\alpha^{3}\right)+g_{2}\left(-1-\alpha^{3}\right)-g_{2}\left(z_{4}^{\prime}\right)\right| \times|\alpha|^{2(k-1)} \\
& \leq\left|g_{1}\left(z_{4}\right)-g_{1}\left(-1-\alpha^{3}\right)\right|+\left|g_{2}\left(-1-\alpha^{3}\right)-g_{2}\left(z_{4}^{\prime}\right)\right| \times|\alpha|^{2(k-1)} \\
& \leq & \left(|\alpha|^{4}+|\alpha|^{3}\right) \times|\alpha|^{2(k-1)} \times \operatorname{diam}(X) \\
& \leq & (1+|\alpha|) \times|\alpha|^{2 k+1} \times \operatorname{diam}(X)
\end{array}
$$

Caso 1.5: Se $a_{i-1}=2, a_{i}=\ldots=a_{k-1}=1, a_{k}=0, a_{k}^{\prime}=1$ e \# $(k-i-1)$ é par então temos as sequências:

$$
\begin{aligned}
t & =\left(a_{1} \ldots 21 \ldots 102222 \ldots\right) \\
t^{\prime} & =\left(a_{1} \ldots 21 \ldots 1100000 \ldots\right)
\end{aligned}
$$

Assim,

$$
\left|f(t)-f\left(t^{\prime}\right)\right|=\left|g_{0} g_{0} g_{2}^{N-k-1}\left(y_{5}\right)-g_{1} g_{0} g_{2}^{N-k-1}\left(y_{5}^{\prime}\right)\right| \times|\alpha|^{2(k-1)} .
$$

Seja $z_{5}=g_{0} g_{2}^{N-k-1}\left(y_{5}\right)$ e $z_{5}^{\prime}=g_{2} g_{0}^{N-k-1}\left(y_{5}^{\prime}\right)$, dai

$$
\left|f(t)-f\left(t^{\prime}\right)\right|=\left|g_{0}\left(z_{5}\right)-g_{1}\left(z_{5}^{\prime}\right)\right| \times|\alpha|^{2(k-1)}
$$


Usando o mesmo argumento temos:

$$
\begin{array}{rlrl}
\left|f(t)-f\left(t^{\prime}\right)\right| & =\left|g_{0}\left(z_{5}\right)-g_{0}\left(-1-\alpha^{3}\right)+g_{1}\left(\alpha^{2}-\alpha^{3}\right)-g_{1}\left(z_{5}^{\prime}\right)\right| \times|\alpha|^{2(k-1)} \\
& \leq\left|g_{0}\left(z_{5}\right)-g_{0}\left(-1-\alpha^{3}\right)\right|+\left|g_{1}\left(\alpha^{2}-\alpha^{3}\right)-g_{1}\left(z_{5}^{\prime}\right)\right| \times|\alpha|^{2(k-1)} \\
& \leq & \left(|\alpha|^{2}+|\alpha|^{3}\right) \times|\alpha|^{2(k-1)} \times \operatorname{diam}(X) \\
& \leq & (1+|\alpha|) \times|\alpha|^{2 k} \times \operatorname{diam}(X)
\end{array}
$$

Caso 1.6: Se $a_{i-1}=2, a_{i}=\ldots=a_{k-1}=1, a_{k}=0, a_{k}^{\prime}=1$ e \# $(k-i-1)$ é impar então temos as sequências:

$$
\begin{aligned}
t & =\left(a_{1} \ldots 21 \ldots 102222 \ldots\right) \\
t^{\prime} & =\left(a_{1} \ldots 21 \ldots 1100000 \ldots\right)
\end{aligned}
$$

Assim,

$$
\left|f(t)-f\left(t^{\prime}\right)\right|=\left|g_{2} g_{0} g_{2}^{N-k-1}\left(y_{6}\right)-g_{1} g_{1} g_{2}^{N-k-1}\left(y_{6}^{\prime}\right)\right| \times|\alpha|^{2(k-1)} .
$$

Seja $z_{6}=g_{0} g_{2}^{N-k-1}\left(y_{6}\right)$ e $z_{6}^{\prime}=g_{1} g_{2}^{N-k-1}\left(y_{6}^{\prime}\right)$, dai

$$
\left|f(t)-f\left(t^{\prime}\right)\right|=\left|g_{2}\left(z_{5}\right)-g_{1}\left(z_{5}^{\prime}\right)\right| \times|\alpha|^{2(k-1)}
$$

Usando o mesmo argumento temos:

$$
\begin{array}{rlrl}
\left|f(t)-f\left(t^{\prime}\right)\right| & = & \left|g_{2}\left(z_{6}\right)-g_{2}\left(-1-\alpha^{3}\right)+g_{1}\left(1-\alpha^{3}\right)-g_{1}\left(z_{6}^{\prime}\right)\right| \times|\alpha|^{2(k-1)} \\
& \leq\left|g_{2}\left(z_{6}\right)-g_{2}\left(-1-\alpha^{3}\right)\right|+\left|g_{1}\left(-1-\alpha^{3}\right)-g_{1}\left(z_{6}^{\prime}\right)\right| \times|\alpha|^{2(k-1)} \\
& \leq & \left(|\alpha|^{4}+|\alpha|^{3}\right) \times|\alpha|^{2(k-1)} \times \operatorname{diam}(X) \\
& \leq & (1+|\alpha|) \times|\alpha|^{2 k+1} \times \operatorname{diam}(X)
\end{array}
$$

Caso 1.7: Se $a_{i-1}=1, a_{i}=\ldots=a_{k-1}=1, a_{k}=0, a_{k}^{\prime}=1 e \#(k-i-1)$ é par então temos as sequências:

$$
\begin{aligned}
t & =\left(a_{1} \ldots 11 \ldots 102222 \ldots\right) \\
t^{\prime} & =\left(a_{1} \ldots 11 \ldots 1100000 \ldots\right)
\end{aligned}
$$

Assim,

$$
\left|f(t)-f\left(t^{\prime}\right)\right|=\left|g_{2} g_{0} g_{2}^{N-k-1}\left(y_{7}\right)-g_{1} g_{0} g_{2}^{N-k-1}\left(y_{7}^{\prime}\right)\right| \times|\alpha|^{2(k-1)} .
$$

Seja $z_{7}=g_{0} g_{2}^{N-k-1}\left(y_{7}\right)$ e $z_{7}^{\prime}=g_{0} g_{2}^{N-k-1}\left(y_{7}^{\prime}\right)$, dai

$$
\left|f(t)-f\left(t^{\prime}\right)\right|=\left|g_{2}\left(z_{7}\right)-g_{1}\left(z_{7}^{\prime}\right)\right| \times|\alpha|^{2(k-1)}
$$


Usando o mesmo argumento temos:

$$
\begin{aligned}
\left|f(t)-f\left(t^{\prime}\right)\right| & = & \left|g_{2}\left(z_{7}\right)-g_{2}\left(-1-\alpha^{3}\right)+g_{1}\left(1-\alpha^{3}\right)-g_{1}\left(z_{7}^{\prime}\right)\right| \times|\alpha|^{2(k-1)} \\
& \leq & \left|g_{2}\left(z_{7}\right)-g_{2}\left(-1-\alpha^{3}\right)\right|+\left|g_{1}\left(-1-\alpha^{3}\right)-g_{1}\left(z_{7}^{\prime}\right)\right| \times|\alpha|^{2(k-1)} \\
& \leq & \left(|\alpha|^{4}+|\alpha|^{3}\right) \times|\alpha|^{2(k-1)} \times \operatorname{diam}(X) \\
& \leq & (1+|\alpha|) \times|\alpha|^{2 k+1} \times \operatorname{diam}(X)
\end{aligned}
$$

Caso 1.8: Se $a_{i-1}=1, a_{i}=\ldots=a_{k-1}=1, a_{k}=0, a_{k}^{\prime}=1$ e \# $(k-i-1)$ é ímpar então temos as sequências:

$$
\begin{aligned}
t & =\left(a_{1} \ldots 11 \ldots 102222 \ldots\right) \\
t^{\prime} & =\left(a_{1} \ldots 11 \ldots 1100000 \ldots\right)
\end{aligned}
$$

Assim,

$$
\left|f(t)-f\left(t^{\prime}\right)\right|=\left|g_{0} g_{0} g_{2}^{N-k-1}\left(y_{8}\right)-g_{1} g_{2} g_{2}^{N-k-1}\left(y_{8}^{\prime}\right)\right| \times|\alpha|^{2(k-1)} .
$$

Seja $z_{8}=g_{0} g_{2}^{N-k-1}\left(y_{8}\right)$ e $z_{8}^{\prime}=g_{2} g_{2}^{N-k-1}\left(y_{8}^{\prime}\right)$, dai

$$
\left|f(t)-f\left(t^{\prime}\right)\right|=\left|g_{0}\left(z_{8}\right)-g_{1}\left(z_{8}^{\prime}\right)\right| \times|\alpha|^{2(k-1)}
$$

Usando o mesmo argumento temos:

$$
\begin{array}{rlrl}
\left|f(t)-f\left(t^{\prime}\right)\right| & =\left|g_{0}\left(z_{8}\right)-g_{0}\left(-1-\alpha^{3}\right)+g_{1}\left(\alpha^{2}-\alpha^{3}\right)-g_{1}\left(z_{8}^{\prime}\right)\right| \times|\alpha|^{2(k-1)} \\
& \leq\left|g_{0}\left(z_{8}\right)-g_{0}\left(-1-\alpha^{3}\right)\right|+\left|g_{1}\left(\alpha^{2}-\alpha^{3}\right)-g_{1}\left(z_{8}^{\prime}\right)\right| \times|\alpha|^{2(k-1)} \\
& \leq & \left(|\alpha|^{2}+|\alpha|^{3}\right) \times|\alpha|^{2(k-1)} \times \operatorname{diam}(X) \\
& \leq & (1+|\alpha|) \times|\alpha|^{2 k} \times \operatorname{diam}(X) .
\end{array}
$$




\section{Referências Bibliográficas}

[1] S. Akiyama, Cubic Pisot units with finite beta expansions, Algebraic Number theory and Diophantine Analysis, (2000), 11-26.

[2] S. Akiyama, Self affine tiling and Pisot numeration system, Number theory and its Applications, (1999), $7-1 \%$

[3] S. Akiyama, On the boundary of self affine tilings generated by Pisot numbers, Journal of Math. Soc. Japan, (2002), 283-308.

[4] P. Arnoux e J.C. Yoccoz, Construction de difféomorphismes pseudo-Anosov, C. R. Acad. Sci. Paris,t. 202 (1981), 75-78.

[5] P. Arnoux, Un exemple de semi-conjugaison entre un échange d'intervalles et une rotation sur le tore, Bull. Soc. Math. France 116 (1998), 489-500.

[6] P. Arnoux e S. Ito, Pisot substitutions and Rauzy fractals, Bull. Belg. Math. Soc. Simon Stevin 8 (2001), 181-20\%.

[7] P. Arnoux, S. Ito e Y. Sano Higher dimensional extensions of substitutions and their dual maps, J. Anal. Math. 83 (2001), 183-206.

[8] P. Arnoux e G. Rauzy, Représentation géométrique de suites de complexité $2 \mathrm{n}+1$, Bull. Soc. Math. France 119 (1991), 101-11\%.

[9] V. Canterini e A. Siegel, Geometric representation of substitutions of Pisot type, Trans. Amer. Math. Soc. 353 (2001), 5121-5144.

[10] F. M. Dekking, Recurrent Sets, Advances in mathematics 44 (1982), 78-104.

[11] K. Falconer, Fractal Geometry, John Wiley-Sons Ltd., Chichester, 1990. Mathematical Foundations and aplications. 
[12] A. S. Fraenkel, Systems of numeration, Amer. Math. Monthly 92 (1985), 105-114.

[13] C. Frougny, Representation of numbers and finite automata, Math. Systems Theory 25 (1992), 37-60. 4, p.713-723.

[14] C. Frougny e B.Solomyak, Finite Beta-expansions, Ergod. Th. and Dynam. Systems 12 (1992), 4, 713-723.

[15] W. J. Gilbert, Complex numbers with three radix expansions, Can. J. Math. xxxIV (1992), $1335-48$.

[16] W. J. Gilbert, Fractal dimension of sets derived from complex bases, Can. Math. Bull., 29 (1986) 495-500.

[17] P. Hubert e A. Messaoudi, Best simultaneous diophantine approximations of Pisot numbers and Rauzy fractals, Acta Arith. 124 (2006), 1-15.

[18] A. Messaoudi, Propriétés arithmétiques et dynamiques du fractal de Rauzy, J. Théor. Nombres Bordeaux 10 (1998), 135-162.

[19] A. Messaoudi, Fronitière du fractal de Rauzy et systèmes de numération complexe, Acta Arithmetica XCV (2000), 195-224.

[20] A. Messaoudi, Combinatorial and geometrical properties of a class of fractals, Bulletin of the Belgian Mathematical Society-Simon Stevin, v. 12, n.4 (2005), 625-633.

[21] A. Messaoudi, Substituições, Fractais e Dinâmica, Tese de Livre Docência UNESP (2008).

[22] N. Pytheas Fogg, Substitutions in dynamics, Arithmetics and Combinatorics, Springer-Verlag, Berlin, 2002. Lecture notes in mathematics, 1794. Edited by: V.Berthé, S. Ferenczi, C. Mauduit, A.Siegel.

[23] W. Parry, On the $\beta$-expansions of real numbers, Acta Math. Acad. Sci. Hungar 11 (1960) $401-416$.

[24] B. Praggastis, Markov partitions for hyperbolic toral automorphism, Ph.d. Thesis, Univ. of Washington (1992).

[25] M. Queffélec, Substitution Dynamical Systems - Spectral Analysis, Lecture Notes in Mathematics, Vol 1294, Springer-Verlag (1987) Berlin.

[26] G. Rauzy, Nombres algébriques et substitutions, Bull. Soc. Math. France 110 (1982) 147-178. 
[27] A. Rényi, Représentations for real numbers and their ergodic properties, Acta. Math. Acad. Sci. Hungar 8 (1957) 477-493.

[28] W. P. Thurston, Groups, tilings, and finite state automata, AMS Colloquim lectures 1 (1990).

[29] S. Ito e M. Mizutani, Potato exchange Transformations with Fractal Domains, Preprint.

[30] S. Ito e M. Kimura, On the Rauzy Fractal, Japan J. Indust. Appl. Math 8 (1991) 461-486.

[31] E. Zeckendorf, Représentation des nombres naturels par une somme de nombres de Fibonacci ou de nombres de Lucas, Bull. Soc. Roy. Sci. liège 41 (1972) 179-182. 